\title{
Global existence and Asymptotic Behavior of the Solutions to Models for Chemotaxis Systems with Chemo Attractants and Repellents
}

Aesha Lagha

West Virginia University, aeshamokhtar@yahoo.com

Follow this and additional works at: https://researchrepository.wvu.edu/etd

Part of the Partial Differential Equations Commons

\section{Recommended Citation}

Lagha, Aesha, "Global existence and Asymptotic Behavior of the Solutions to Models for Chemotaxis Systems with Chemo Attractants and Repellents" (2020). Graduate Theses, Dissertations, and Problem Reports. 7965.

https://researchrepository.wvu.edu/etd/7965

This Dissertation is protected by copyright and/or related rights. It has been brought to you by the The Research Repository @ WVU with permission from the rights-holder(s). You are free to use this Dissertation in any way that is permitted by the copyright and related rights legislation that applies to your use. For other uses you must obtain permission from the rights-holder(s) directly, unless additional rights are indicated by a Creative Commons license in the record and/ or on the work itself. This Dissertation has been accepted for inclusion in WVU Graduate Theses, Dissertations, and Problem Reports collection by an authorized administrator of The Research Repository @ WVU.

For more information, please contact researchrepository@mail.wvu.edu. 
Global existence and Asymptotic Behavior of the Solutions to Models for Chemotaxis Systems with Chemo Attractants and Repellents

\author{
Aesha Lagha \\ Dissertation submitted \\ to the Eberly College of Arts and Sciences \\ at West Virginia University \\ in partial fulfillment of the requirements for the degree of \\ Doctor of Philosophy in \\ Mathematics
}

\author{
Harumi Hattori, Ph.D., Chair \\ Adrian Tudorascu, Ph.D. \\ Adam Halasz, Ph.D. \\ Casian Pantea, Ph.D. \\ Tudor Stanescu, Ph.D. \\ Department of Mathematics
}

Morgantown, West Virginia

2020

Keywords: energy method, a priori estimates, Fourier transform, time-decay rate

Copyright 2020 Aesha Lagha 


\begin{abstract}
Global existence and Asymptotic Behavior of the Solutions to Models for Chemotaxis Systems with Chemo Attractants and Repellents
\end{abstract}

\title{
Aesha Lagha
}

We study global existence and asymptotic behavior of the solutions to models for chemotaxis systems with chemo attractants and repellents in three dimensions. Chemo attractants and repellents may be called chemo agents. For Part I, we use the logistic model for the mass. The interactions between chemo agents and the mass are taken into account. For Part II, we consider the case when mass is conserved and we use the Lotka-Volterra type model for chemo agents. To accomplish this, we use the Fourier transform and energy method. We show the existence of global solutions by the energy method. Also, we establish $L^{q}$ timedecay for the linear homogeneous system by using the Fourier transform and finding Green's matrix. Then, we find $L^{q}$ time-decay for the nonlinear system using solution representation by Duhamel's principle and time-weighted estimates. 
Table of Contents

Page

Cover Page ......................... i

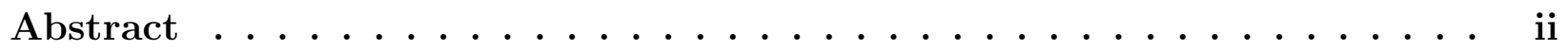

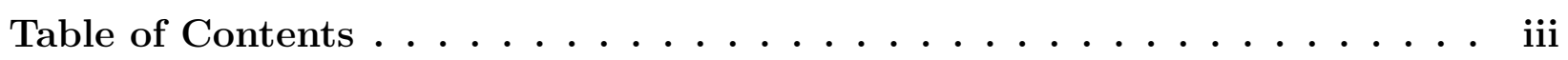

Acknowledgments ........................ v v

I Existence of Global Solutions to Chemotaxis Fluid System with Logistic Source 1

Chapter 1: Introduction .................... 2

Chapter 2: Reformulation of the system $(1.1) \ldots \ldots \ldots \ldots$

Chapter 3: Global solution of the nonlinear system $(2.2) \ldots \ldots \ldots$

3.0 .1 Existence of local solutions . . . . . . . . . . . . . . . 8

$3.0 .2 \quad$ A Priori Estimates . . . . . . . . . . . . . . . . . 16

Chapter 4: Linearized homogeneous system . . . . . . . . . . . 21

4.0 .1 Representation of solutions . . . . . . . . . . . . . . 22

$4.0 .2 \quad L^{2}-L^{q}$ time-decay property $\ldots \ldots \ldots \ldots \ldots \ldots \ldots$

Chapter 5: Time-Decay Rates for the Nonlinear System _ . . . . . . 31

5.0.1 Time rate for the energy functional and high-order energy functional 31

5.0 .2 Time-decay rate in $L^{q} \ldots \ldots \ldots \ldots$ 


\section{Global Existence and Decay Rates of the Solutions for a}

Chemotaxis System with Lotka-Volterra Type Model for Chemo Agents

Chapter 6: Introduction . . . . . . . . . . . . . 40

Chapter 7: Reformulation of the system $(6.3) \ldots \ldots \ldots \ldots 4$

Chapter 8: Global solution of the nonlinear system $(7.2) \ldots \ldots$. . . . 47

8.0 .1 Existence of local solutions . . . . . . . . . . . . . . . . 47

8.0 .2 A Priori Estimates . . . . . . . . . . . . . . . . . . 48

Chapter 9: Linearized Homogeneous System _ . . . . . . . . . . 55

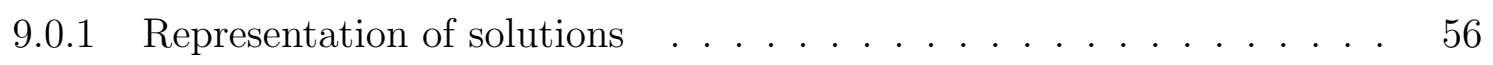

9.0.2 Refined $L^{2}-L^{q}$ time-decay property. . . . . . . . . . . . 61

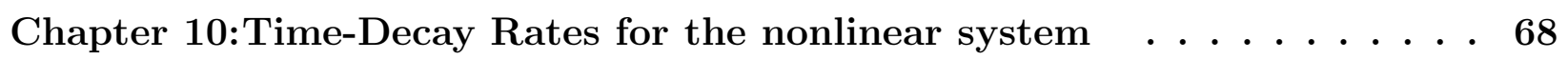

10.0.1 Decay rates for the energy functional and high-order energy functional 68

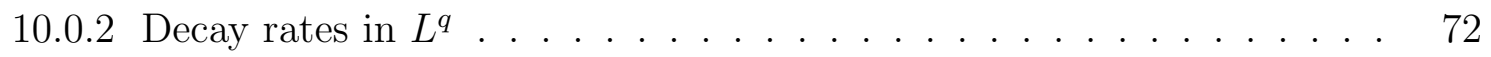

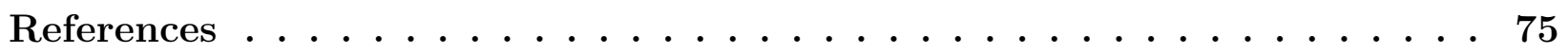




\section{Acknowledgements}

First and foremost I wish to thank my advisor, Professor Harumi Hattori. He has taught me and supported me academically to finish this dissertation. Also, I would like to thank my committee members. I must express my gratitude to my husband, for his continued encouragement. Most of all, I want to acknowledge and thank my parents, sisters and brothers. 
Part I

\title{
Existence of Global Solutions to
}

Chemotaxis Fluid System with

\author{
Logistic Source
}




\section{Chapter 1}

\section{Introduction}

Chemotaxis is the oriented movement of biological cells or microscopic organisms toward the concentration gradient of certain chemicals in their environment. This type of movement exists in many biological phenomena, such as the movement of bacteria toward certain chemicals [1], or the movement of endothelial cells toward the higher concentration of chemoattractant that cancer cells produce [2]. Keller and Segel [3],[4] established some mathematical models to describe the aggregation of certain types of bacteria. Since then, many mathematical approaches to describe chemotaxis using systems of partial differential equations have emerged. In this paper, we use the equations for continuum mechanics to describe the movement of bacteria or the growth of blood vessels, and for the chemoattractant and repellent, we use diffusion equations. The combined effects of chemoattractant and repellent for chemotaxis are studied in diseases, such as Alzheimer's disease.

We consider the initial value problem of the Chemotaxis system in $\mathbb{R}^{3}$ taking the following form:

$$
\left\{\begin{array}{l}
\partial_{t} n+\nabla \cdot(n u)=n\left(n_{\infty}-n\right) \\
\partial_{t} u+u \cdot \nabla u+\frac{\nabla p(n)}{n}=\nabla c_{1}^{2}-\nabla c_{2}^{2}+\delta \Delta u \\
\partial_{t} c_{1}=\Delta c_{1}-a_{12} c_{1}+a_{11} n c_{1} \\
\partial_{t} c_{2}=\Delta c_{2}-a_{22} c_{2}+a_{21} n c_{2} .
\end{array}\right.
$$

Here, $n(x, t), u(x, t), c_{1}(x, t), c_{2}(x, t)$ and $p(n)$ for $t>0, x \in \mathbb{R}^{3}$, are the cell concentration, velocity of cells, chemoattractant concentration, chemorepellent concentration, and pressure of the cells, respectively. Constant $\delta$ is the corresponding coefficient for the viscosity term. 
The initial data is given by

$$
\left.\left(n, u, c_{1}, c_{2}\right)\right|_{t=0}=\left(n_{0}, u_{0}, c_{1,0}, c_{2,0}\right)(x), \quad x \in \mathbb{R}^{3}
$$

where it is supposed to hold that

$$
\left(n_{0}, u_{0}, c_{1,0}, c_{2,0}\right)(x) \rightarrow\left(n_{\infty}, 0,0,0\right) \text { as }|x| \rightarrow \infty
$$

for some constant $n_{\infty}>0$. Throughout this paper, we assume the following: $\delta>0, p($. is the smooth function of $n$ and $p^{\prime}(n)>0$. The main goal of this paper is to establish the local and global existence of smooth solutions in three dimensions around a constant state $\left(n_{\infty}, 0,0,0\right)$ and the decay rate of global smooth solutions for the above system (1.1). The main result of this paper is stated as follows.

Theorem 1.0.1. Let $N \geq 4$ be an integer. There exists a positive numbers $\epsilon_{0}, C_{0}$ such that if

$$
\left\|\left[n_{0}-n_{\infty}, u_{0}, c_{1,0}, c_{2,0}\right]\right\|_{H^{N}} \leq \epsilon_{0}
$$

then, the Cauchy problem (1.1)-(1.2) has a unique solution $\left(n, u, c_{1}, c_{2}\right)(t)$ globally in time which satisfies

$$
\begin{aligned}
& \left(u, c_{1}, c_{2}\right)(t) \in C\left([0, \infty) ; H^{N}\left(\mathbb{R}^{3}\right)\right) \cap C^{1}\left([0, \infty) ; H^{N-2}\left(\mathbb{R}^{3}\right)\right), \\
& n-n_{\infty} \in C\left([0, \infty) ; H^{N}\left(\mathbb{R}^{3}\right)\right) \cap C^{1}\left([0, \infty) ; H^{N-1}\left(\mathbb{R}^{3}\right)\right)
\end{aligned}
$$

and there are constants $\lambda_{1}>0$ and $\lambda_{2}>0$ such that

$$
\begin{aligned}
\left\|\left[n-n_{\infty}, u, c_{1}, c_{2}\right]\right\|_{H^{N}}^{2} & +\lambda_{1} \int_{0}^{t}\left\|\nabla\left[u, c_{1}, c_{2}\right]\right\|_{H^{N}}^{2} \\
& +\lambda_{2} \int_{0}^{t}\left\|\left[n-n_{\infty}, c_{1}, c_{2}\right]\right\|_{H^{N}}^{2} \\
& \leq C_{0}\left\|\left[n_{0}-n_{\infty}, u_{0}, c_{1,0}, c_{2,0}\right]\right\|_{H^{N}}^{2}
\end{aligned}
$$

Furthermore, the global solution $\left[n, u, c_{1}, c_{2}\right]$ satisfies the following time-decay rates for $t \geq 0$ :

$$
\begin{gathered}
\left\|\left(n-n_{\infty}\right)\right\|_{L^{q}} \leq C(1+t)^{-2+\frac{3}{2 q}} \\
\|u\|_{L^{q}} \leq C(1+t)^{\frac{-3}{2}+\frac{3}{2 q}}
\end{gathered}
$$




$$
\left\|\left(c_{1}, c_{2}\right)\right\|_{L^{q}} \leq C(1+t)^{\frac{-3}{2}}
$$

with $2 \leq q<\infty, C>0$.

The proof of the existence of global solutions in Theorem 1.0.1 is based on the local existence and an a priori estimates. We show the local solutions by constructing a sequence of approximation functions based on iteration. To obtain a priori estimates we use the energy method. Moreover, to obtain the time-decay rate in $L^{q}$ norm of solutions in Theorem 1.0.1, our approach is a combined analysis of Green's function of the linear system and the refined energy estimates with the help of Duhamel's principle. We obtain Green's matrix of the linear system by using the Fourier transform.

To motivate our study, we present some previous related works about chemotaxis models. Such chemotaxis models are based on the Keller-Segel system. Wang [5] explored the interactions between the nonlinear diffusion and logistic source on the solutions of the attraction-repulsion chemotaxis system in three dimensions. E. Lankeit and J. Lankeit [6] proved the global existence of classical solutions to a chemotaxis system with singular sensitivity. Liu and Wang [7] established the existence of global classical solutions and steady states to an attraction-repulsion chemotaxis model in one dimension based on the method of energy estimates.

Concerning the chemotaxis models based on fluid dynamics, there are two approaches, incompressible and compressible. For the incompressible case, Chae, Kang and Lee [8], and Duan, Lorz, and Markowich [9] showed the global-in-time existence for the incompressible chemotaxis equations near the constant states, if the initial data is sufficiently small. Rodriguez, Ferreira, and Villamizar-Roa [10] showed the global existence for an attractionrepulsion chemotaxis fluid model with logistic source. Tan and Zhou [11] proved the global existence and time decay estimate of solutions to the Keller- Segel system in $R^{3}$ with the small initial data. For the compressible case, Ambrosi, Bussolino, and Preziosi [12] discussed the vasculogenesis using the compressible fluid dynamics for the cells and the diffusion equation for the attractant.

Many related approaches use the Fourier transform, and we only mention that Duan [13] and Duan, Liu, and Zhu [14] proved the time-decay rate by the combination of energy 
estimates and spectral analysis.

For later use in this paper, we give some notations. C denotes some positive constant and $\lambda_{i}$, where $i=1,2$, denotes some positive (generally small) constant, where both $\mathrm{C}$ and $\lambda_{i}$ may take different values in different places. For any integer $m \geq 0$, we use $H^{m}$ to denote the Sobolev space $H^{m}\left(\mathbb{R}^{3}\right)$. Set $L^{2}=H^{0}$. We set $\partial^{\alpha}=\partial_{x_{1}}^{\alpha_{1}} \partial_{x_{2}}^{\alpha_{2}} \partial_{x_{3}}^{\alpha_{3}}$ for a multiindex $\alpha=\left[\alpha_{1}, \alpha_{2}, \alpha_{3}\right]$. The length of $\alpha$ is $||=.\alpha_{1}+\alpha_{2}+\alpha_{3}$; we also set $\partial_{j}=\partial_{x_{j}}$ for $j=1,2,3$. For an integrable function $f: \mathbb{R}^{3} \rightarrow \mathbb{R}$, its Fourier transform is defined by $\hat{f}=\int_{\mathbb{R}^{3}} e^{-i x \cdot \xi} f(x) d x, x \cdot \xi=\sum_{i=0}^{3} x_{j} \xi_{j}$, and $x \in \mathbb{R}^{3}$, where $i=\sqrt{-1}$ is the imaginary unit. Let us denote the space

$$
\begin{aligned}
X(0, T)= & \left\{\left(u, c_{1}, c_{2}\right) \in C\left([0, T] ; H^{N}\left(\mathbb{R}^{3}\right)\right) \cap C^{1}\left([0, T] ; H^{N-2}\left(\mathbb{R}^{3}\right)\right),\right. \\
& \left.n-n_{\infty} \in C\left([0, T] ; H^{N}\left(\mathbb{R}^{3}\right)\right) \cap C^{1}\left([0, T] ; H^{N-1}\left(\mathbb{R}^{3}\right)\right)\right\} .
\end{aligned}
$$

This paper is organized as follows. In chapter 2, we reformulate the Cauchy problem under consideration. In chapter 3, we prove the global existence and uniqueness of solutions. In chapter 4 , we investigate the linearized homogeneous system to obtain the $L^{2}-L^{q}$ timedecay property and the explicit representation of solutions. In chapter 5 , we study the $L^{q}$ time-decay rates of solutions to the reformulated nonlinear system and finish the proof of Theorem1.0.1. 


\section{Chapter 2}

\section{Reformulation of the system (1.1)}

Let $U(t)=\left[n, u, c_{1}, c_{2}\right]$ be a smooth solution to the Cauchy problem of the chemotaxis system (1.1) with initial data $U_{0}=\left[n_{0}, u_{0}, c_{1,0}, c_{2,0}\right]$. We introduce the transformation:

$$
n(x, t)=n_{\infty}+\rho(x, t) .
$$

Then the Cauchy problem (1.1) is reformulated as

$$
\left\{\begin{array}{l}
\partial_{t} \rho+n_{\infty} \nabla \cdot u+n_{\infty} \rho=-\nabla \cdot(\rho u)-\rho^{2} \\
\partial_{t} u+u \cdot \nabla u-\delta \Delta u+\frac{p^{\prime}\left(n_{\infty}\right)}{n_{\infty}} \nabla \rho=\nabla c_{1}^{2}-\nabla c_{2}^{2}-\left(\frac{p^{\prime}\left(\rho+n_{\infty}\right)}{\rho+n_{\infty}}-\frac{p^{\prime}\left(n_{\infty}\right)}{n_{\infty}}\right) \nabla \rho \\
\partial_{t} c_{1}=\Delta c_{1}-\left(a_{12}-a_{11} n_{\infty}\right) c_{1}+a_{11} \rho c_{1} \\
\partial_{t} c_{2}=\Delta c_{2}-\left(a_{22}-a_{21} n_{\infty}\right) c_{2}+a_{21} \rho c_{2}
\end{array}\right.
$$

with initial data

$$
\left.\left(\rho, u, c_{1}, c_{2}\right)\right|_{t=0}=\left(\rho_{0}, u_{0}, c_{1,0}, c_{2,0}\right) \rightarrow(0,0,0,0)
$$

as $|x| \rightarrow \infty$, where $\rho_{0}=n_{0}-n_{\infty}$. We assume that $a_{12}-a_{11} n_{\infty}>0$ and $a_{22}-a_{21} n_{\infty}>0$. In what follows, the integer $N \geq 4$ is always assumed.

Proposition 2.0.1. There exists a positive number $\epsilon_{0}$ which is small enough such that if

$$
\left\|\left[\rho_{0}, u_{0}, c_{1,0}, c_{2,0}\right]\right\|_{H^{N}} \leq \epsilon_{0}
$$

then the Cauchy problem (2.2)-(2.3) has a unique solution $\left(\rho, u, c_{1}, c_{2}\right)(t)$ globally in time which satisfies $\left(\rho, u, c_{1}, c_{2}\right)(t) \in X(0, \infty)$ and there are constants $C_{0}>0, \lambda_{1}>0$ and $\lambda_{1}>0$ 
such that

$$
\begin{array}{r}
\left\|\left[\rho, u, c_{1}, c_{2}\right]\right\|_{H^{N}}^{2}+\lambda_{1} \int_{0}^{t}\left\|\nabla\left[u, c_{1}, c_{2}\right]\right\|_{H^{N}}^{2}+\lambda_{2} \int_{0}^{t}\left\|\left[\rho, c_{1}, c_{2}\right]\right\|_{H^{N}}^{2} \\
\leq C_{0}\left\|\left[\rho_{0}, u_{0}, c_{1,0}, c_{2,0}\right]\right\|_{H^{N}}^{2} .
\end{array}
$$

Proposition 2.0.2. Let $U(t)=\left[\rho, u, c_{1}, c_{2}\right]$ be the solution to the Cauchy problem (2.2)-(2.3) obtained in Proposition 2.0.1, which satisfies the following $L^{q}$-time decay estimates for any $t \geq 0$ :

$$
\begin{gathered}
\|\rho\|_{L^{q}} \leq C(1+t)^{-2+\frac{3}{2 q}}, \\
\|u\|_{L^{q}} \leq C(1+t)^{\frac{-3}{2}+\frac{3}{2 q}}, \\
\left\|\left(c_{1}, c_{2}\right)\right\|_{L^{q}} \leq C(1+t)^{\frac{-3}{2}},
\end{gathered}
$$

with $2 \leq q<\infty$ and $C>0$.

The proof of Theorem 1.0.1 obtained directly from the global existence proof in Proposition 2.0.1 and the derivation of rates in Theorem 1.0.1 is based on Proposition 2.0.2. 


\section{Chapter 3}

\section{Global solution of the nonlinear}

\section{system (2.2)}

The goal of this chapter is to prove the global existence of solutions to the Cauchy problem (2.2) when initial data is a small, smooth perturbation near the steady state $\left(n_{\infty}, 0,0,0\right)$. The proof is based on some uniform a priori estimates combined with the local existence, which will be shown in subsections 3.0.1 and 3.0.2.

\subsubsection{Existence of local solutions}

In this subsection, we show the proof of the existence of local solutions $\left[\rho, u, c_{1}, c_{2}\right]$ by constructing a sequence of functions that converges to a function satisfying the Cauchy problem. We construct a solution sequence $\left(\rho^{j}, u^{j}, c_{1}^{j}, c_{2}^{j}\right)_{j \geq 0}$ by iteratively solving the Cauchy problem on the following

$$
\left\{\begin{array}{l}
\partial_{t} \rho^{j+1}+n_{\infty} \nabla \cdot u^{j+1}+n_{\infty} \rho^{j+1}=-\rho^{j} \nabla \cdot u^{j+1}-\nabla \rho^{j+1} u^{j}-\rho^{j^{2}} \\
\partial_{t} u^{j+1}-\delta \Delta u^{j+1}=-u^{j} \cdot \nabla u^{j}+\nabla c_{1}^{j^{2}}-\nabla c_{2}^{j^{2}}-\frac{p^{\prime}\left(\rho^{j}+n_{\infty}\right)}{\rho^{j}+n_{\infty}} \nabla \rho^{j} \\
\partial_{t} c_{1}^{j+1}-\Delta c_{1}^{j+1}+\left(a_{12}-a_{11} n_{\infty}\right) c_{1}^{j+1}=a_{11} \rho^{j} c_{1}^{j+1} \\
\partial_{t} c_{2}^{j+1}-\Delta c_{2}^{j+1}+\left(a_{22}-a_{21} n_{\infty}\right) c_{2}^{j+1}=a_{21} \rho^{j} c_{2}^{j+1},
\end{array}\right.
$$

with initial data

$$
\left.\left(\rho^{j+1}, u^{j+1}, c_{1}^{j+1}, c_{2}^{j+1}\right)\right|_{t=0}=U_{0}=\left(\rho_{0}, u_{0}, c_{1,0}, c_{2,0}\right) \rightarrow(0,0,0,0)
$$


as $|x| \rightarrow \infty$, for $j \geq 0$. For simplicity, in what follows, we write $U^{j}=\left(\rho^{j}, u^{j}, c_{1}^{j}, c_{2}^{j}\right)$ and $U_{0}=\left(\rho_{0}, u_{0}, c_{1,0}, c_{2,0}\right)$, where $U^{0}=(0,0,0,0)$.

Now, we can start the following Lemma.

Lemma 3.0.1. There are constants $T_{1}$ and $\epsilon_{0}>0$ such that if the initial data $U_{0} \in H^{N}\left(\mathbb{R}^{3}\right)$ and $\left\|U_{0}\right\|_{H^{N}} \leq \epsilon_{0}$, then there exists a unique solution $U=\left(\rho, u, c_{1}, c_{2}\right)$ of the Cauchy problem (2.2)-(2.3) on $\left[0, T_{1}\right]$ with $U \in X\left(0, T_{1}\right)$.

Proof. We first set $U^{0}=(0,0,0,0)$. Then, we use $U^{0}$ to solve the equations for $U^{1}$. The first equation is the first order partial differential equation and the second, third, and fourth equations are the second order parabolic equations. We obtain $u^{1}(x, t), c_{1}^{1}(x, t), c_{2}^{1}(x, t)$, and $\rho^{1}(x, t)$ in this order. Similarly, we define $\left(u^{j}, c_{1}^{j}, c_{2}^{j}, \rho^{j}\right)$ iteratively. Now, we prove the existence and uniqueness of solutions in space $C\left(\left[0, T_{1}\right] ; H^{N}\left(\mathbb{R}^{3}\right)\right)$, where $T_{1}>0$ is suitably small. The proof is divided into four steps as follows.

In the first step, we show the uniform boundedness of the sequence of functions under our construction via energy estimates. We show that there exists a constant $M>0$ such that $U^{j} \in C\left(\left[0, T_{1}\right] ; H^{N}\left(\mathbb{R}^{3}\right)\right)$ is well defined and

$$
\sup _{0 \leq t \leq T_{1}}\left\|U^{j}(t)\right\|_{H^{N}} \leq M
$$

for all $j \geq 0$. We use the induction to prove (3.3). It is trivial when $j=0$. Suppose that it is true for $j \geq 0$ where $M$ is small enough. To prove for $j+1$, we need some energy estimate for $U^{j+1}$. Applying $\partial^{\alpha}$ to the first equation of (3.1), multiplying it by $\partial^{\alpha} \rho^{j+1}$ and integrating in $x$, we obtain

$$
\begin{array}{r}
\frac{1}{2} \frac{d}{d t} \int_{\mathbb{R}^{3}}\left(\partial^{\alpha} \rho^{j+1}\right)^{2} \mathrm{~d} x+n_{\infty} \int_{\mathbb{R}^{3}}\left|\partial^{\alpha} \rho^{j+1}\right|^{2} \mathrm{~d} x=-n_{\infty} \int_{\mathbb{R}^{3}} \partial^{\alpha} \rho^{j+1} \partial^{\alpha} \nabla \cdot u^{j+1} \mathrm{~d} x \\
-\int_{\mathbb{R}^{3}} \partial^{\alpha} \rho^{j+1} \partial^{\alpha}\left(\nabla \rho^{j+1} u^{j}\right) \mathrm{d} x+\int_{\mathbb{R}^{3}} \partial^{\alpha} \rho^{j+1} \partial^{\alpha}\left(\rho^{j} \nabla \cdot u^{j+1}\right) \mathrm{d} x-\int_{\mathbb{R}^{3}} \partial^{\alpha} \rho^{j+1} \partial^{\alpha} \rho^{j 2} \mathrm{~d} x .
\end{array}
$$

The terms on the right hand side are further bounded by

$$
\begin{array}{r}
C\left\|\nabla \cdot u^{j+1}\right\|_{H^{N}}\left\|\rho^{j+1}\right\|_{H^{N}}+C\left\|\nabla \cdot u^{j}\right\|_{L^{\infty}}\left\|\rho^{j+1}\right\|_{H^{N}}^{2}+\left\|u^{j}\right\|_{H^{N}}\left\|\rho^{j+1}\right\|_{H^{N}}\left\|\nabla \rho^{j+1}\right\|_{H^{N-2}} \\
\left\|\rho^{j}\right\|_{H^{N}}\left\|\rho^{j+1}\right\|_{H^{N}}\left\|\nabla \cdot u^{j+1}\right\|_{H^{N}}+C\left\|\rho^{j}\right\|_{H^{N-2}}\left\|\rho^{j+1}\right\|_{H^{N}}\left\|\rho^{j}\right\|_{H^{N}} .
\end{array}
$$


Then, after taking the summation over $|\alpha| \leq N$ and using the Cauchy inequality, one has

$$
\begin{aligned}
\frac{1}{2} \frac{d}{d t}\left\|\rho^{j+1}\right\|_{H^{N}}^{2} & +\lambda_{2}\left\|\rho^{j+1}\right\|_{H^{N}}^{2} \leq C\left\|\nabla \cdot u^{j+1}\right\|_{H^{N}}^{2}+C\left\|u^{j}\right\|_{H^{N}}^{2}\left\|\rho^{j+1}\right\|_{H^{N}}^{2} \\
& +C\left\|\rho^{j}\right\|_{H^{N}}^{2}\left\|\rho^{j+1}\right\|_{H^{N}}^{2}+C\left\|\rho^{j}\right\|_{H^{N}}^{2} .
\end{aligned}
$$

Similarly, applying $\partial^{\alpha}$ to the second equation of (3.1), multiplying it by $\partial^{\alpha} u^{j+1}$, taking integrations in $x$, and then using integration by parts, we have

$$
\begin{aligned}
\frac{1}{2} \frac{d}{d t} \int_{\mathbb{R}^{3}}\left(\partial^{\alpha} u^{j+1}\right)^{2} \mathrm{~d} x+\delta \int_{\mathbb{R}^{3}}\left|\partial^{\alpha} \nabla \cdot u^{j+1}\right|^{2} \mathrm{~d} x=\frac{p^{\prime}\left(n_{\infty}\right)}{n_{\infty}} \int_{\mathbb{R}^{3}} \nabla \cdot \partial^{\alpha} u^{j+1} \partial^{\alpha} \rho^{j+1} \mathrm{~d} x \\
-\int_{\mathbb{R}^{3}} \nabla \cdot \partial^{\alpha} u^{j+1} \partial^{\alpha} c_{1}^{j^{2}} \mathrm{~d} x+\int_{\mathbb{R}^{3}} \nabla \cdot \partial^{\alpha} u^{j+1} \partial^{\alpha} c_{2}^{j^{2}} \mathrm{~d} x \\
-\int_{\mathbb{R}^{3}} \partial^{\alpha} u^{j+1} \partial^{\alpha}\left(u^{j} \cdot \nabla u^{j}\right) \mathrm{d} x-\int_{\mathbb{R}^{3}} \partial^{\alpha} u^{j+1} \partial^{\alpha}\left(\frac{\nabla p\left(\rho^{j}+n_{\infty}\right)}{\rho^{j}+n_{\infty}}\right) \mathrm{d} x
\end{aligned}
$$

Then, after taking the summation over $|\alpha| \leq N$, the terms on the right side of the previous equation are bounded by

$$
\begin{gathered}
C\left\|\nabla \cdot u^{j+1}\right\|_{H^{N}}\left\|\rho^{j+1}\right\|_{H^{N}}+C\left\|c_{1}^{j}\right\|_{H^{N-3}}\left\|\nabla \cdot u^{j+1}\right\|_{H^{N}}\left\|c_{1}^{j}\right\|_{H^{N}}+C\left\|c_{2}^{j}\right\|_{H^{N-3}}\left\|\nabla \cdot u^{j+1}\right\|_{H^{N}}\left\|c_{2}^{j}\right\|_{H^{N}} \\
+\left\|u^{j}\right\|_{H^{N}}^{2}\left\|\nabla \cdot u^{j+1}\right\|_{H^{N}}+C\left\|\rho^{j}\right\|_{H^{N}}\left\|\nabla \cdot u^{j+1}\right\|_{H^{N}} .
\end{gathered}
$$

By using the Cauchy inequality, we obtain

$$
\begin{gathered}
\frac{1}{2} \frac{d}{d t}\left\|u^{j+1}\right\|_{H^{N}}^{2}+ \\
\lambda_{1}\left\|\nabla \cdot u^{j+1}\right\|_{H^{N}}^{2} \leq C\left\|\rho^{j+1}\right\|_{H^{N}}^{2}+C\left\|c_{1}^{j}\right\|_{H^{N}}^{2}+C\left\|c_{1}^{j}\right\|_{H^{N}}^{2}\left\|\nabla \cdot u^{j+1}\right\|_{H^{N}}^{2}+C\left\|c_{2}^{j}\right\|_{H^{N}}^{2} \\
+C\left\|c_{2}^{j}\right\|_{H^{N}}^{2}\left\|\nabla \cdot u^{j+1}\right\|_{H^{N}}^{2}+C\left\|u^{j}\right\|_{H^{N}}^{2}\left\|\nabla \cdot u^{j+1}\right\|_{H^{N}}^{2}+\left\|\rho^{j}\right\|_{H^{N}}^{2} .
\end{gathered}
$$

In a similar way as above, we can estimate $c_{1}$ and $c_{2}$ as

$$
\begin{aligned}
& \frac{1}{2} \frac{d}{d t}\left\|c_{1}^{j+1}\right\|_{H^{N}}^{2}+\left\|\nabla c_{1}^{j+1}\right\|_{H^{N}}^{2}+\lambda_{2}\left\|c_{1}^{j+1}\right\|_{H^{N}}^{2} \leq C\left\|\rho^{j}\right\|_{H^{N}}^{2}\left\|c_{1}^{j+1}\right\|_{H^{N}}^{2} \\
& \frac{1}{2} \frac{d}{d t}\left\|c_{2}^{j+1}\right\|_{H^{N}}^{2}+\left\|\nabla c_{2}^{j+1}\right\|_{H^{N}}^{2}+\lambda_{2}\left\|c_{2}^{j+1}\right\|_{H^{N}}^{2} \leq C\left\|\rho^{j}\right\|_{H^{N}}^{2}\left\|c_{2}^{j+1}\right\|_{H^{N}}^{2} .
\end{aligned}
$$

Taking the linear combination of inequalities (3.4)-(3.7), we have

$$
\begin{aligned}
& \frac{1}{2} \frac{d}{d t}\left(\left\|\rho^{j+1}\right\|_{H^{N}}^{2}+\left\|u^{j+1}\right\|_{H^{N}}^{2}+\left\|c_{1}^{j+1}\right\|_{H^{N}}^{2}+\left\|c_{2}^{j+1}\right\|_{H^{N}}^{2}\right)+\lambda_{1}\left\|\nabla\left[u^{j+1}, c_{1}^{j+1}, c_{2}^{j+1}\right]\right\|_{H^{N}}^{2} \\
& +\lambda_{2}\left\|\left[\rho^{j+1}, c_{1}^{j+1}, c_{2}^{j+1}\right]\right\|_{H^{N}}^{2} \leq C\left\|\left[\rho^{j}, u^{j}, c_{1}^{j}, c_{2}^{j}\right]\right\|_{H^{N}}^{2}+C\left\|\left[\rho^{j}, u^{j}\right]\right\|_{H^{N}}^{2}\left\|\rho^{j+1}\right\|_{H^{N}}^{2} \\
& +C\left\|\left[u^{j}, c_{1}^{j}, c_{2}^{j}\right]\right\|_{H^{N}}^{2}\left\|\nabla \cdot u^{j+1}\right\|_{H^{N}}^{2}+C\left\|\rho^{j}\right\|_{H^{N}}^{2}\left\|\left[c_{1}^{j+1}, c_{2}^{j+1}\right]\right\|_{H^{N}}^{2} .
\end{aligned}
$$


Thus, after integrating with respect to $t$, we have

$$
\begin{aligned}
& \left\|U^{j+1}(t)\right\|_{H^{N}}^{2}+\lambda_{1} \int_{0}^{t}\left\|\nabla\left[u^{j+1}, c_{1}^{j+1}, c_{2}^{j+1}\right]\right\|_{H^{N}}^{2} \mathrm{~d} s+\lambda_{2} \int_{0}^{t}\left\|\left[\rho^{j+1}, c_{1}^{j+1}, c_{2}^{j+1}\right]\right\|_{H^{N}}^{2} \mathrm{~d} s \\
& \leq C\left\|U^{j+1}(0)\right\|_{H^{N}}^{2}+C \int_{0}^{t}\left\|U^{j}(s)\right\|_{H^{N}}^{2} \mathrm{~d} s+C \int_{0}^{t}\left\|U^{j}(s)\right\|_{H^{N}}^{2}\left\|\left[\rho^{j+1}, \nabla \cdot u^{j+1}, c_{1}^{j+1}, c_{2}^{j+1}\right]\right\|_{H^{N}}^{2} \mathrm{~d} s .
\end{aligned}
$$

In the last inequality, we use the induction hypothesis. We obtain

$$
\begin{array}{r}
\left\|U^{j+1}(t)\right\|_{H^{N}}^{2}+\lambda_{1} \int_{0}^{t}\left\|\nabla\left[u^{j+1}, c_{1}^{j+1}, c_{2}^{j+1}\right]\right\|_{H^{N}}^{2} \mathrm{~d} s+\lambda_{2} \int_{0}^{t}\left\|\left[\rho^{j+1}, c_{1}^{j+1}, c_{2}^{j+1}\right]\right\|_{H^{N}}^{2} \mathrm{~d} s \\
\leq C \epsilon_{0}^{2}+C M^{2} T_{1}+C M^{2} \int_{0}^{t}\left\|\left[\rho^{j+1}, \nabla \cdot u^{j+1}, c_{1}^{j+1}, c_{2}^{j+1}\right]\right\|_{H^{N}}^{2} \mathrm{~d} s
\end{array}
$$

for $0 \leq t \leq T_{1}$. Now, we take the small constants $\epsilon_{0}>0, T_{1}>0$ and $M>0$. Then we have

$$
\left\|U^{j+1}(t)\right\|_{H^{N}}^{2}+\lambda_{1} \int_{0}^{t}\left\|\nabla\left[u^{j+1}, c_{1}^{j+1}, c_{2}^{j+1}\right]\right\|_{H^{N}}^{2} \mathrm{~d} s+\lambda_{2} \int_{0}^{t}\left\|\left[\rho^{j+1}, c_{1}^{j+1}, c_{2}^{j+1}\right]\right\|_{H^{N}}^{2} \mathrm{~d} s \leq M^{2}
$$

for $0 \leq t \leq T_{1}$. This implies that (3.3) holds true for $j+1$. Hence (3.3) is proved for all $j \geq 0$.

For the second step, we prove that the sequence $\left(U^{j}\right)_{j \geq 0}$ is a Cauchy sequence in the Banach space $C\left(\left[0, T_{1}\right] ; H^{N-1}\left(\mathbb{R}^{3}\right)\right)$, which converges to the solution $U=\left(\rho, u, c_{1}, c_{2}\right)$ of the Cauchy problem (2.2)-(2.3), and satisfies $\sup _{0 \leq t \leq T_{1}}\left\|\left[U^{j}(t)\right]\right\|_{H^{N-1}} \leq M$. See for example [15].

For simplicity, we denote $\delta f^{j+1}:=f^{j+1}-f^{j}$. Subtracting the $j$-th equations from the $(j+1)$-th equations, we have the following equations for $\delta \rho^{j+1}, \delta u^{j+1}, \delta c_{1}^{j+1}$ and $\delta c_{1}^{j+1}$ :

$$
\left\{\begin{array}{l}
\partial_{t} \delta \rho^{j+1}+n_{\infty} \nabla \cdot\left(\delta u^{j+1}\right)+n_{\infty} \delta \rho^{j+1}=-\rho^{j} \nabla \cdot \delta u^{j+1}-\delta \rho^{j} \nabla \cdot u^{j} \\
-u^{j} \nabla \delta \rho^{j+1}-\delta u^{j} \nabla \rho^{j}+\left(\rho^{j}+\rho^{j-1}\right) \delta \rho^{j} \\
\partial_{t} \delta u^{j+1}-\delta \Delta \delta u^{j+1}=-u^{j} \cdot \nabla \delta u^{j}-\delta u^{j} \cdot \nabla u^{j-1}+\nabla\left(\left(c_{1}^{j}+c_{1}^{j-1}\right) \delta c_{1}^{j}\right) \\
-\nabla\left(\left(c_{2}^{j}+c_{2}^{j-1}\right) \delta c_{2}^{j}\right)-\left(\frac{\nabla p\left(\rho^{j}+n_{\infty}\right)}{\rho^{j}+n_{\infty}}-\frac{\nabla p\left(\rho^{j-1}+n_{\infty}\right)}{\rho^{j-1}+n_{\infty}}\right) \\
\partial_{t} \delta c_{1}^{j+1}+\Delta \delta c_{1}^{j+1}+\left(a_{12}-a_{11} n_{\infty}\right) \delta c_{1}^{j+1}=a_{11} \rho^{j} \delta c_{1}^{j+1}+a_{11} \delta \rho^{j} c_{1}^{j} \\
\partial_{t} \delta c_{2}^{j+1}+\Delta \delta c_{2}^{j+1}+\left(a_{22}-a_{21} n_{\infty}\right) \delta c_{2}^{j+1}=a_{21} \rho^{j} \delta c_{2}^{j+1}+a_{21} \delta \rho^{j} c_{2}^{j} .
\end{array}\right.
$$

The estimate of $\delta \rho^{j+1}$ is as follows: 


$$
\begin{aligned}
\frac{1}{2} \frac{d}{d t} & \left\|\delta \rho^{j+1}\right\|_{H^{N-1}}^{2}+n_{\infty}\left\|\delta \rho^{j+1}\right\|_{H^{N-1}}^{2} \leq C\left\|\nabla \cdot \delta u^{j+1}\right\|_{H^{N-1}}\left\|\delta \rho^{j+1}\right\|_{H^{N-1}} \\
& +C\left\|\rho^{j}\right\|_{H^{N-1}}\left\|\delta \rho^{j+1}\right\|_{H^{N-1}}\left\|\nabla \cdot \delta u^{j+1}\right\|_{H^{N-1}}+C\left\|\delta \rho^{j}\right\|_{H^{N-1}}\left\|\nabla \cdot u^{j}\right\|_{H^{N-1}}\left\|\delta \rho^{j+1}\right\|_{H^{N-1}} \\
& +C\left\|\nabla \cdot u^{j}\right\|_{L^{\infty}}\left\|\delta \rho^{j+1}\right\|_{H^{N-1}}^{2}+C\left\|\delta \rho^{j+1}\right\|_{H^{N-2}}\left\|u^{j}\right\|_{H^{N-1}}\left\|\delta \rho^{j+1}\right\|_{H^{N-1}} \\
& +C\left\|\delta \rho^{j+1}\right\|_{H^{N-1}}\left\|\delta u^{j}\right\|_{H^{N-1}}\left\|\nabla \rho^{j}\right\|_{H^{N-1}}+C\left\|\delta \rho^{j+1}\right\|_{H^{N-1}}\left\|\delta \rho^{j}\right\|_{H^{N-1}} .
\end{aligned}
$$

Then

$$
\begin{aligned}
\frac{1}{2} \frac{d}{d t} & \left\|\delta \rho^{j+1}\right\|_{H^{N-1}}^{2}+\lambda_{2}\left\|\delta \rho^{j+1}\right\|_{H^{N-1}}^{2} \leq C\left\|\nabla \cdot \delta u^{j+1}\right\|_{H^{N-1}}^{2}+C\left\|\rho^{j}\right\|_{H^{N-1}}^{2}\left\|\delta \rho^{j+1}\right\|_{H^{N-1}}^{2} \\
& +C\left\|\nabla \cdot u^{j}\right\|_{H^{N-1}}^{2}\left\|\delta \rho^{j}\right\|_{H^{N-1}}^{2}+C\left\|u^{j}\right\|_{H^{N-1}}^{2}\left\|\delta \rho^{j+1}\right\|_{H^{N-1}}^{2} \\
& +C\left\|\nabla \rho^{j}\right\|_{H^{N-1}}^{2}\left\|\delta u^{j}\right\|_{H^{N-1}}^{2}+C\left\|\delta \rho^{j}\right\|_{H^{N-1}}^{2}
\end{aligned}
$$

The estimate of $\delta u^{j+1}$ is

$$
\begin{aligned}
& \frac{1}{2} \frac{d}{d t}\left\|\delta u^{j+1}\right\|_{H^{N-1}}^{2}+\delta\left\|\nabla \cdot \delta u^{j+1}\right\|_{H^{N-1}}^{2} \leq C\left\|\nabla \cdot \delta u^{j+1}\right\|_{H^{N-1}}\left\|u^{j}\right\|_{H^{N-1}}\left\|\delta u^{j}\right\|_{H^{N-1}} \\
& +\left\|\delta u^{j+1}\right\|_{H^{N-1}}\left\|\nabla \cdot u^{j}\right\|_{H^{N-1}}\left\|\delta u^{j}\right\|_{H^{N-1}}+C\left\|\delta u^{j+1}\right\|_{H^{N-1}}\left\|\delta u^{j}\right\|_{H^{N-1}}\left\|\nabla \cdot u^{j-1}\right\|_{H^{N-1}} \\
& +C\left\|\delta c_{1}^{j}\right\|_{H^{N-1}}^{2}\left\|\nabla \cdot \delta u^{j+1}\right\|_{H^{N-1}}^{2}+C\left\|\delta c_{2}^{j+1}\right\|_{H^{N-1}}^{2}\left\|\nabla \cdot \delta u^{j+1}\right\|_{H^{N-1}}^{2}+C\left\|\delta \rho^{j+1}\right\|_{H^{N}}^{2} \\
& +C\left\|\delta \rho^{j}\right\|_{H^{N-1}}\left\|\nabla \cdot \delta u^{j+1}\right\|_{H^{N-1}} .
\end{aligned}
$$

Then

$$
\begin{aligned}
\frac{1}{2} \frac{d}{d t} \| & \delta u^{j+1}\left\|_{H^{N-1}}^{2}+\lambda_{1}\right\| \nabla \cdot \delta u^{j+1} \|_{H^{N-1}}^{2} \\
& \leq C\left\|u^{j}\right\|_{H^{N-1}}^{2}\left\|\delta u^{j}\right\|_{H^{N-1}}^{2}+\left\|\delta u^{j+1}\right\|_{H^{N-1}}^{2}\left\|\nabla \cdot u^{j}\right\|_{H^{N-1}}^{2} \\
& +C\left\|\delta u^{j+1}\right\|_{H^{N-1}}^{2}\left\|\nabla \cdot u^{j-1}\right\|_{H^{N-1}}^{2}+C\left\|\delta u^{j}\right\|_{H^{N-1}}^{2} \\
& +C\left\|\delta c_{1}^{j}\right\|_{H^{N-1}}^{2}+C\left\|\delta c_{2}^{j}\right\|_{H^{N-1}}^{2}+\left\|\delta \rho^{j}\right\|_{H^{N-1}}^{2}
\end{aligned}
$$

We have a similar way to estimate $\delta c_{1}^{j+1}$ and $\delta c_{2}^{j+1}$ as follows:

$$
\begin{aligned}
\frac{1}{2} \frac{d}{d t}\left\|\delta c_{1}^{j+1}\right\|_{H^{N-1}}^{2} & +\left\|\nabla \delta c_{1}^{j+1}\right\|_{H^{N-1}}^{2}+\lambda_{2}\left\|\delta c_{1}^{j+1}\right\|_{H^{N-1}}^{2} \\
& \leq C\left\|\rho^{j}\right\|_{H^{N-1}}^{2}\left\|\delta c_{1}^{j+1}\right\|_{H^{N-1}}^{2}+C\left\|\delta c_{1}^{j}\right\|_{H^{N-1}}^{2}\left\|\rho^{j}\right\|_{H^{N-1}}^{2}
\end{aligned}
$$


and

$$
\begin{aligned}
\frac{1}{2} \frac{d}{d t}\left\|\delta c_{2}^{j+1}\right\|_{H^{N-1}}^{2} & +\left\|\nabla \delta c_{2}^{j+1}\right\|_{H^{N-1}}^{2}+\lambda_{2}\left\|\delta c_{2}^{j+1}\right\|_{H^{N-1}}^{2} \\
& \leq C\left\|\rho^{j}\right\|_{H^{N-1}}^{2}\left\|\delta c_{2}^{j+1}\right\|_{H^{N-1}}^{2}+C\left\|\delta c_{2}^{j}\right\|_{H^{N-1}}^{2}\left\|\rho^{j}\right\|_{H^{N-1}}^{2}
\end{aligned}
$$

We combine the equations (3.10)-(3.13) to obtain

$$
\begin{aligned}
& \frac{1}{2} \frac{d}{d t}\left(\left\|\delta \rho^{j+1}\right\|_{H^{N-1}}^{2}+\left\|\delta u^{j+1}\right\|_{H^{N-1}}^{2}+\left\|\delta c_{1}^{j+1}\right\|_{H^{N-1}}^{2}+\left\|\delta c_{2}^{j+1}\right\|_{H^{N-1}}^{2}\right) \\
& +\lambda_{1}\left(\left\|\nabla \cdot \delta u^{j+1}\right\|_{H^{N-1}}^{2}+\left\|\nabla \delta c_{1}^{j+1}\right\|_{H^{N-1}}^{2}+\left\|\nabla \delta c_{2}^{j+1}\right\|_{H^{N-1}}^{2}\right) \\
& +\lambda_{2}\left(\left\|\delta \rho^{j+1}\right\|_{H^{N-1}}^{2}+\left\|\delta c_{1}^{j+1}\right\|_{H^{N-1}}^{2}+C\left\|\delta c_{2}^{j+1}\right\|_{H^{N-1}}^{2}\right) \\
& \leq C\left(\left\|\delta u^{j+1}\right\|_{H^{N-1}}^{2}+\left\|\delta \rho^{j+1}\right\|_{H^{N-1}}^{2}+\left\|\delta c_{1}^{j+1}\right\|_{H^{N-1}}^{2}+\left\|\delta c_{2}^{j+1}\right\|_{H^{N-1}}^{2}\right) \\
& +C\left(\left\|\delta u^{j}\right\|_{H^{N-1}}^{2}+C\left\|\delta \rho^{j}\right\|_{H^{N-1}}^{2}+C\left\|\delta c_{1}^{j}\right\|_{H^{N-1}}^{2}+C\left\|\delta c_{2}^{j}\right\|_{H^{N-1}}^{2}\right) .
\end{aligned}
$$

By using Gronwall's inequality, we obtain

$$
\begin{aligned}
\sup _{0 \leq t \leq T_{1}}\left(\left\|\delta \rho^{j+1}\right\|_{H^{N-1}}^{2}+\left\|\delta u^{j+1}\right\|_{H^{N-1}}^{2}+\right. & \left.\left\|\delta c_{1}^{j+1}\right\|_{H^{N-1}}^{2}+\left\|\delta c_{2}^{j+1}\right\|_{H^{N-1}}^{2}\right) \\
& \leq e^{\int_{0}^{t} c \mathrm{~d} s \int_{0}^{t}\left\|\delta U^{j}(s)\right\|_{H^{N-1}}^{2} d s+e^{\int_{0}^{t} c \mathrm{~d} s}\left\|\delta U^{j+1}(0)\right\|_{H^{N-1}}^{2} d s} \\
& \leq C T_{1}\left(e^{C T_{1}}\right) \sup _{0 \leq t \leq T_{1}}\left\|\delta U^{j}\right\|_{H^{N-1}}^{2} .
\end{aligned}
$$

By taking $T_{1}>0$ sufficiently small we find that $\left(U^{j}\right)_{j \geq 0}$ is a Cauchy sequence in the Banach space $C\left(\left[0, T_{1}\right] ; H^{N-1}\left(\mathbb{R}^{3}\right)\right)$. Thus, we have the limit function

$$
U=U^{0}+\lim _{m \rightarrow \infty} \sum_{j=0}^{m}\left(U^{j+1}-U^{j}\right)
$$

in the same space $C\left(\left[0, T_{1}\right] ; H^{N-1}\left(\mathbb{R}^{3}\right)\right)$, and satisfies

$$
\sup _{0 \leq t \leq T_{1}}\|U\|_{H^{N-1}} \leq \sup _{0 \leq t \leq T_{1}} \lim _{j \rightarrow \infty} \inf \left\|U^{j}\right\|_{H^{N-1}} \leq M
$$

Thus, as $j \rightarrow \infty$ the limit exists such that

$$
(U)_{j \geq 0} \rightarrow U(t)
$$

strongly in $C\left(\left[0, T_{1}\right] ; H^{N-1}\right)$ and as $j^{\prime} \rightarrow \infty$, where $\left\{j^{\prime}\right\}$ is a subsequence of $\{j\}$, we have

$$
D\left(u, c_{1}, c_{2}\right)_{j^{\prime}} \rightarrow D\left(u, c_{1}, c_{2}\right)
$$


weakly in $L_{2}\left(\left[0, T_{1}\right] ; H^{N}\right)$ by step one. Also by step one, we know

$$
(U)_{j^{\prime \prime}}(t) \rightarrow U(t)
$$

weakly in $H^{N}$ for every fixed $t \in\left[0, T_{1}\right]$, where $j^{\prime \prime}=j^{\prime \prime}(t)$ is a subsequence of $\left\{j^{\prime}\right\}$, depending on $t$. Thus, we have a solution $U(t) \in L_{\infty}\left(\left[0, T_{1}\right] ; H^{N}\right)$ for the problem (2.2)-(2.3). For the third step, we show that $\left\|U^{j+1}(t)\right\|_{H^{N}}^{2}$ is continuous in time for each $j \geq 0$. For simplicity, let us define the equivalent energy functional

$$
\mathfrak{E}\left(U^{j+1}(t)\right)=\left\|\rho^{j+1}\right\|_{H^{N}}^{2}+\left\|u^{j+1}\right\|_{H^{N}}^{2}+\left\|c_{1}^{j+1}\right\|_{H^{N}}^{2}+\left\|c_{2}^{j+1}\right\|_{H^{N}}^{2} .
$$

Similarly to how we proved (3.8), we have

$$
\begin{aligned}
& \left|\mathfrak{E} U^{j+1}(t)-\mathfrak{E} U^{j+1}(s)\right|=\left|\int_{s}^{t} \mathfrak{E} U^{j+1}(\theta) \mathrm{d} \theta\right| \leq \int_{s}^{t}\left\|U^{j}(s)\right\|_{H^{N}}^{2} \mathrm{~d} \theta \\
& +C \int_{0}^{t}\left(1+\left\|U^{j}(s)\right\|_{H^{N}}^{2}\right)\left\|\left[\rho^{j+1}, \nabla \cdot u^{j+1}, c_{1}^{j+1}, c_{2}^{j+1}\right]\right\|_{H^{N}}^{2} \mathrm{~d} s+C \int_{s}^{t}\left\|\nabla\left[c_{1}^{j+1}, c_{2}^{j+1}\right]\right\|_{H^{N}}^{2} \mathrm{~d} s \\
& \leq C M^{2}(t-s)+C\left(M^{2}+1\right) \int_{s}^{t}\left\|\left[\rho^{j+1}, \nabla \cdot u^{j+1}, c_{1}^{j+1}, c_{2}^{j+1}\right]\right\|_{H^{N}}^{2} \mathrm{~d} s \\
& +C \int_{s}^{t}\left\|\nabla\left[c_{1}^{j+1}, c_{2}^{j+1}\right]\right\|_{H^{N}}^{2} \mathrm{~d} s
\end{aligned}
$$

for any $0 \leq s \leq t \leq T_{1}$. The time integral on the right-hand side from the above inequality is bounded by (3.9), and hence $\mathfrak{E} U^{j+1}(t)$ is continuous in $t$ for each $j \geq 0$. Therefore, $\left\|U^{j}(t)\right\|_{H^{N}}^{2}$ is continuous in time for each $j \geq 1$.

Furthermore, $U=\left(\rho, u, c_{1}, c_{2}\right)$ is a local solution to the Cauchy problem (2.2)-(2.3).

For the fourth step, we show that the Cauchy problem (2.2)-(2.3) admits at most one solution in $C\left(\left[0, T_{1}\right] ; H^{N}\left(\mathbb{R}^{3}\right)\right)$. We assume that there exist two local solutions $U, \tilde{U}$ in $C\left(\left[0, T_{1}\right] ; H^{N}\right)$ which satisfiy $(3.2)$. Let $\tilde{\rho}=\rho_{1}(x, t)-\rho_{2}(x, t), \tilde{u}(x, t)=u_{1}(x, t)-u_{2}(x, t), \quad \tilde{c}_{1}(x, t)=$ $c_{1,1}(x, t)-c_{1,2}(x, t)$ and $\tilde{c}_{2}(x, t)=c_{2,1}(x, t)-c_{2,2}(x, t)$ solve

$$
\left\{\begin{array}{l}
\partial_{t} \tilde{\rho}+n_{\infty} \nabla \cdot \tilde{u}+n_{\infty} \tilde{\rho}=-\nabla \cdot\left(\tilde{\rho} u_{1}\right)-\nabla \cdot\left(\rho_{2} \tilde{u}\right)-\left(\rho_{1}+\rho_{2}\right) \tilde{\rho} \\
\partial_{t} \tilde{u}+u_{1} \cdot \nabla \tilde{u}-\delta \Delta \tilde{u}=-\tilde{u} \cdot \nabla u_{2}-\frac{p^{\prime}\left(\rho_{1}+n_{\infty}\right)}{\rho_{1}+n_{\infty}} \nabla \tilde{\rho}+\nabla\left(\left(c_{1,1}+c_{1,2}\right) \tilde{c}_{1}\right) \\
-\nabla\left(\left(c_{2,1}+c_{2,2}\right)\right) \tilde{c}_{2}-\left(\frac{p^{\prime}\left(\rho_{1}+n_{\infty}\right)}{\rho_{1}+n_{\infty}}-\frac{p^{\prime}\left(\rho_{2}+n_{\infty}\right)}{\rho_{2}+n_{\infty}}\right) \nabla \rho_{2} \\
\partial_{t} \tilde{c}_{1}=\Delta \tilde{c}_{1}-a_{12} \tilde{c}_{1}+a_{11} \rho_{1} \tilde{c}_{1}+a_{11} \tilde{\rho}_{1} c_{1,2} \\
\partial_{t} \tilde{c}_{2}=\Delta \tilde{c}_{2}-a_{22} \tilde{c}_{2}+a_{21} \rho_{1} \tilde{c}_{2}+a_{21} \tilde{\rho} c_{2,2} .
\end{array}\right.
$$


Multiplying $\tilde{\rho}$ to both sides of the first equation of (3.15) and integrating over $\mathbb{R}^{3}$, we have

$$
\int_{\mathbb{R}^{3}} \tilde{\rho} \partial_{t} \tilde{\rho} d x+n_{\infty} \int_{\mathbb{R}^{3}} \tilde{\rho} \nabla \cdot \tilde{u} d x+n_{\infty} \int_{\mathbb{R}^{3}}|\tilde{\rho}|^{2} d x=-\int_{\mathbb{R}^{3}} \tilde{\rho} \nabla \cdot\left(\tilde{\rho} u_{1}\right) d x+\int_{\mathbb{R}^{3}} \tilde{\rho} \nabla \cdot\left(\rho_{2} \tilde{u}\right) d x+\int_{\mathbb{R}^{3}}\left(\rho_{1}+\rho_{2}\right) \tilde{\rho}^{2} .
$$

Using integration by parts and the Cauchy-Schwarz inequality, we have

$$
\begin{aligned}
\frac{1}{2} \frac{d}{d t}\|\tilde{\rho}\|_{L^{2}}^{2}+ & n_{\infty}\|\tilde{\rho}\|_{L^{2}}^{2} \leq \frac{n_{\infty}}{2}\|\tilde{\rho}\|_{L^{2}}^{2}+\frac{n_{\infty}}{2}\|\nabla \cdot \tilde{u}\|_{L^{2}}^{2}+\frac{1}{2}\left\|\nabla \cdot u_{1}\right\|_{L^{\infty}} \int_{\mathbb{R}^{3}}|\tilde{\rho}|^{2} d x \\
& +\left\|\rho_{2}\right\|_{L^{\infty}} \int_{\mathbb{R}^{3}}\left(|\nabla \cdot \tilde{u}|^{2}+|\tilde{\rho}|^{2}\right) d x+\left\|\nabla \rho_{2}\right\|_{L^{\infty}} \int_{\mathbb{R}^{3}}\left(|\tilde{u}|^{2}+|\tilde{\rho}|^{2}\right) d x \\
& +\left\|\left[\rho_{1}+\rho_{2}\right]\right\|_{L^{\infty}} \int_{\mathbb{R}^{3}}|\tilde{\rho}|^{2} d x .
\end{aligned}
$$

Next, we establish the energy estimates for $\tilde{u}$. By multiplying $\tilde{u}$ to both sides of the second equation of (3.15) and integrating in $x$, we have

$$
\begin{aligned}
\int_{\mathbb{R}^{3}} \tilde{u} \partial_{t} \tilde{u} d x & +\int_{\mathbb{R}^{3}} \tilde{u} u_{1} \cdot \nabla \tilde{u} d x-\delta \int_{\mathbb{R}^{3}} \tilde{u} \Delta \tilde{u} d x=-\int_{\mathbb{R}^{3}} \tilde{u} \cdot \nabla u_{2} d x+\int_{\mathbb{R}^{3}} \frac{p^{\prime}\left(n_{\infty}\right)}{n_{\infty}} \tilde{u} \nabla \tilde{\rho} d x \\
& +\int_{\mathbb{R}^{3}} \tilde{u}\left(\frac{p^{\prime}\left(\rho_{1}+n_{\infty}\right)}{\rho_{1}+n_{\infty}}-\frac{p^{\prime}\left(n_{\infty}\right)}{n_{\infty}}\right) \nabla \tilde{\rho}+\int_{\mathbb{R}^{3}} \tilde{u} \nabla\left(\left(c_{1,1}+c_{1,2}\right) \tilde{c}_{1}\right) d x \\
& -\int_{\mathbb{R}^{3}} \tilde{u} \nabla\left(\left(c_{2,1}+c_{2,2}\right) \tilde{c}_{2}\right) d x-\int_{\mathbb{R}^{3}} \tilde{u}\left(\frac{p^{\prime}\left(\rho_{1}+n_{\infty}\right)}{\rho_{1}+n_{\infty}} d x-\frac{p^{\prime}\left(\rho_{2}+n_{\infty}\right)}{\rho_{2}+n_{\infty}}\right) \nabla \rho_{2} d x .
\end{aligned}
$$

By using integration by parts and the Cauchy-Schwarz inequality, we have

$$
\begin{aligned}
\frac{1}{2} \frac{d}{d t}\|\tilde{u}\|_{L^{2}}^{2} & +\delta\|\nabla \cdot \tilde{u}\|_{L^{2}}^{2} \leq\left\|\nabla \cdot u_{1}\right\|_{L^{\infty}}\|\tilde{u}\|_{L^{2}}^{2}+\left\|\nabla \cdot u_{2}\right\|_{L^{\infty}}\|\tilde{u}\|_{L^{2}}^{2}+\frac{p^{\prime}\left(n_{\infty}\right)}{2 n_{\infty}}\|\nabla \cdot \tilde{u}\|_{L^{2}}^{2}+\frac{p^{\prime}\left(n_{\infty}\right)}{2 n_{\infty}}\|\tilde{\rho}\|_{L^{2}}^{2} \\
& +\left\|\rho_{1}\right\|_{L^{\infty}}\left(\|\nabla \cdot \tilde{u}\|_{L^{2}}^{2}+\|\tilde{\rho}\|_{L^{2}}^{2}\right)+\left\|\nabla \rho_{1}\right\|_{L^{\infty}}\left(\|\tilde{u}\|_{L^{2}}^{2}+\|\tilde{\rho}\|_{L^{2}}^{2}\right) \\
& +\left\|c_{1,1}+c_{1,2}\right\|_{L^{\infty}}\left(\|\nabla \cdot \tilde{u}\|_{L^{2}}^{2}+\left\|\tilde{c}_{1}\right\|_{L^{2}}^{2}\right)+\left\|c_{2,1}+c_{2,2}\right\|_{L^{\infty}}\left(\|\nabla \cdot \tilde{u}\|_{L^{2}}^{2}+\left\|\tilde{c}_{2}\right\|_{L^{2}}^{2}\right) \\
& +\left\|\nabla \rho_{2}\right\|_{L^{\infty}}\left(\|\tilde{u}\|_{L^{2}}^{2}+\|\tilde{\rho}\|_{L^{2}}^{2}\right) .
\end{aligned}
$$

Since $L^{\infty}$ norms of $\rho_{i}, u_{i}, c_{1, i}, c_{2, i}$ where $i=1,2$ are bounded, we have

$$
\frac{1}{2} \frac{d}{d t}\|\tilde{u}\|_{L^{2}}^{2}+\frac{\delta}{2}\|\nabla \cdot \tilde{u}\|_{L^{2}}^{2} \leq C\|\tilde{u}\|_{L^{2}}^{2}+C\|\tilde{\rho}\|_{L^{2}}^{2}+C\left\|\tilde{c}_{1}\right\|_{L^{2}}^{2}+C\left\|\tilde{c}_{2}\right\|_{L^{2}}^{2} .
$$

We have a similar way to estimate $\tilde{c}_{1}$ and $\tilde{c}_{2}$ as follows:

$$
\begin{aligned}
& \frac{1}{2} \frac{d}{d t}\left\|\tilde{c}_{1}\right\|_{L^{2}}^{2}+\left\|\nabla \tilde{c}_{1}\right\|_{L^{2}}^{2}+a_{12}\left\|\tilde{c}_{1}\right\|_{L^{2}}^{2} \leq a_{11}\left\|\rho_{1}\right\|_{L^{\infty}}\left\|\tilde{c}_{1}\right\|_{L^{2}}^{2}+\frac{a_{11}}{2}\left\|c_{1,2}\right\|_{L^{\infty}}\left(\|\tilde{\rho}\|_{L^{2}}^{2}+\left\|\tilde{c}_{1}\right\|_{L^{2}}^{2}\right) \\
& \frac{1}{2} \frac{d}{d t}\left\|\tilde{c}_{2}\right\|_{L^{2}}^{2}+\left\|\nabla \tilde{c}_{2}\right\|_{L^{2}}^{2}+a_{22}\left\|\tilde{c}_{2}\right\|_{L^{2}}^{2} \leq a_{21}\left\|\rho_{1}\right\|_{L^{\infty}}\left\|\tilde{c}_{2}\right\|_{L^{2}}^{2}+\frac{a_{21}}{2}\left\|c_{2,2}\right\|_{L^{\infty}}\left(\|\tilde{\rho}\|_{L^{2}}^{2}+\left\|\tilde{c}_{2}\right\|_{L^{2}}^{2}\right) .
\end{aligned}
$$


By taking a linear combination of all estimates, we obtain

$$
\begin{aligned}
\frac{1}{2} \frac{d}{d t}\left(\|\tilde{\rho}\|_{L^{2}}^{2}+\|\tilde{u}\|_{L^{2}}^{2}+\left\|\tilde{c}_{1}\right\|_{L^{2}}^{2}+\left\|\tilde{c}_{2}\right\|_{L^{2}}^{2}\right)+\lambda_{1}\left(\|\nabla \cdot \tilde{u}\|_{L^{2}}^{2}+\left\|\tilde{\nabla} c_{1}\right\|_{L^{2}}^{2}+\left\|\tilde{\nabla} c_{2}\right\|_{L^{2}}^{2}\right) \\
+\lambda_{2}\left(\|\tilde{\rho}\|_{L^{2}}^{2}+\left\|\tilde{c}_{1}\right\|_{L^{2}}^{2}+\left\|\tilde{c}_{2}\right\|_{L^{2}}^{2}\right) \leq C\left(\|\tilde{\rho}\|_{L^{2}}^{2}+\|\tilde{u}\|_{L^{2}}^{2}+\left\|\tilde{c}_{1}\right\|_{L^{2}}^{2}+\left\|\tilde{c}_{2}\right\|_{L^{2}}^{2}\right) .
\end{aligned}
$$

By applying Gronwall's inequality to the above equation, we have

$$
\sup _{0 \leq t \leq T_{1}}\left(\|\tilde{\rho}\|_{L^{2}}^{2}+\|\tilde{u}\|_{L^{2}}^{2}+\left\|\tilde{c}_{1}\right\|_{L^{2}}^{2}+\left\|\tilde{c}_{2}\right\|_{L^{2}}^{2}\right) \leq e^{c T_{1}}\left(\|\tilde{\rho}(0)\|_{L^{2}}^{2}+\|\tilde{u}(0)\|_{L^{2}}^{2}+\left\|\tilde{c}_{1}(0)\right\|_{L^{2}}^{2}+\left\|\tilde{c}_{2}(0)\right\|_{L^{2}}^{2}\right) .
$$

Since the initial data of $\left(\tilde{\rho}, \tilde{u}, \tilde{c}_{1}, \tilde{c}_{2}\right)$ are all zero for $T>0$, that implies the uniqueness of the local solution.

\subsubsection{A Priori Estimates}

In this subsection, we provide some estimates for the solutions for any $t>0$. We use the energy method to obtain uniform-in-time a priori estimates for smooth solutions to Cauchy problems (2.2)-(2.3).

Lemma 3.0.2. (a priori estimates) Let $U(t)=\left(\rho, u, c_{1}, c_{2}\right) \in C\left([0, T] ; H^{N}\left(\mathbb{R}^{3}\right)\right.$ be the smooth solution to the Cauchy problem (2.2)-(2.3) for $T>0$ with

$$
\sup _{0 \leq t \leq T}\left\|\left(\rho, u, c_{1}, c_{2}\right)(t)\right\|_{N} \leq \epsilon
$$

for $0<\epsilon \leq 1$. Then, there are $\epsilon_{0}>0, C_{0}>0, \lambda_{1}>0$ and $\lambda_{2}>0$ such that for any $\epsilon \leq \epsilon_{0}$

$$
\begin{aligned}
\left\|\left[\rho, u, c_{1}, c_{2}\right]\right\|_{H^{N}}^{2}+\lambda_{1} \int_{0}^{t}\left\|\nabla\left[u, c_{1}, c_{2}\right]\right\|_{H^{N}}^{2}+ & \lambda_{2} \int_{0}^{t}\left\|\left[\rho, c_{1}, c_{2}\right]\right\|_{H^{N}}^{2} \\
& \leq C_{0}\left\|\left[\rho_{0}, u_{0}, c_{1,0}, c_{2,0}\right]\right\|_{H^{N}}^{2}
\end{aligned}
$$

holds for any $t \in[0, T]$.

Proof. First, we find the zero-order estimates. For the estimate of $\rho$, multiplying $\rho$ to both sides of the first equation of (2.2) and taking integrations in $x \in \mathbb{R}^{3}$, we obtain

$$
\int_{\mathbb{R}^{3}} \rho \rho_{t} \mathrm{~d} x+n_{\infty} \int_{\mathbb{R}^{3}} \rho \nabla \cdot u \mathrm{~d} x+n_{\infty} \int_{\mathbb{R}^{3}}|\rho|^{2} \mathrm{~d} x=-\int_{\mathbb{R}^{3}} \rho \nabla \cdot(\rho u) \mathrm{d} x-\int_{\mathbb{R}^{3}} \rho \rho^{2} \mathrm{~d} x .
$$


Using integration by parts and the Cauchy-Schwarz inequality, we have

$$
\begin{aligned}
\frac{1}{2} \int_{\mathbb{R}^{3}}\left(\rho^{2}\right)_{t} \mathrm{~d} x+n_{\infty} \int_{\mathbb{R}^{3}}|\rho|^{2} \mathrm{~d} x+ & n_{\infty} \int_{\mathbb{R}^{3}} \rho \nabla \cdot u \mathrm{~d} x \\
& \leq \frac{1}{2} \sup _{x}|\nabla u| \int_{\mathbb{R}^{3}}|\rho|^{2} \mathrm{~d} x+\sup _{x}|\rho| \int_{\mathbb{R}^{3}}|\rho|^{2} \mathrm{~d} x \\
& \leq C\|\rho, u\|_{H^{N}} \int_{\mathbb{R}^{3}}|\rho|^{2} \mathrm{~d} x .
\end{aligned}
$$

Now, we estimate $u$ by multiplying the second equation of (2.2) by $u$ and integrating over $\mathbb{R}^{3}$. Then, we have

$$
\begin{aligned}
\int_{\mathbb{R}^{3}} u u_{t} \mathrm{~d} x+\int_{\mathbb{R}^{3}} u(u \cdot \nabla u) \mathrm{d} x-\delta & \int_{\mathbb{R}^{3}} u \Delta u \mathrm{~d} x+\frac{p^{\prime}\left(n_{\infty}\right)}{n_{\infty}} \int_{\mathbb{R}^{3}} u \cdot \nabla \rho \mathrm{d} x=\int_{\mathbb{R}^{3}} u \nabla c_{1}^{2} \mathrm{~d} x \\
& -\int_{\mathbb{R}^{3}} u \nabla c_{2}^{2} \mathrm{~d} x-\int_{\mathbb{R}^{3}} u\left(\frac{p^{\prime}\left(\rho+n_{\infty}\right)}{\rho+n_{\infty}}-\frac{p^{\prime}\left(n_{\infty}\right)}{n_{\infty}}\right) \nabla \rho \mathrm{d} x .
\end{aligned}
$$

By using integration by parts and the Cauchy-Schwarz inequality, we have

$$
\begin{aligned}
\frac{1}{2} \int_{\mathbb{R}^{3}}\left(u^{2}\right)_{t} \mathrm{~d} x & +\delta \int_{\mathbb{R}^{3}}|\nabla u|^{2} \mathrm{~d} x-\frac{p^{\prime}\left(n_{\infty}\right)}{n_{\infty}} \int_{\mathbb{R}^{3}} \rho \nabla \cdot u \mathrm{~d} x \\
& \leq\|u\|_{H^{1}} \int_{\mathbb{R}^{3}}|\nabla u|^{2} \mathrm{~d} x+C\|u\|_{H^{N}} \int_{\mathbb{R}^{3}}\left(\left|c_{1}\right|^{2}+\left|c_{2}\right|^{2}+|\rho|^{2}\right) \mathrm{d} x .
\end{aligned}
$$

For the estimates of $c_{1}$, we multiply $c_{1}$ to both sides of the equation of $c_{1}$ and integrate with respect to $x$, and we have

$$
\int_{\mathbb{R}^{3}} c_{1}\left(c_{1}\right)_{t} \mathrm{~d} x-\int_{\mathbb{R}^{3}} c_{1} \Delta c_{1} \mathrm{~d} x+\left(a_{12}-n_{\infty} a_{11}\right) \int_{\mathbb{R}^{3}}\left|c_{1}\right|^{2} \mathrm{~d} x \leq a_{11} \sup _{x}|\rho| \int_{\mathbb{R}^{3}}\left|c_{1}\right|^{2} \mathrm{~d} x .
$$

By using integration by parts, we have

$$
\frac{1}{2} \int_{\mathbb{R}^{3}}\left(c_{1}^{2}\right)_{t} \mathrm{~d} x+\int_{\mathbb{R}^{3}}\left|\nabla c_{1}\right|^{2} \mathrm{~d} x+\left(a_{12}-n_{\infty} a_{11}\right) \int_{\mathbb{R}^{3}}\left|c_{1}\right|^{2} \mathrm{~d} x \leq a_{11}\|\rho\|_{H^{2}} \int_{\mathbb{R}^{3}}\left|c_{1}\right|^{2} \mathrm{~d} x .
$$

Similar to above, from the equation of $c_{2}$, we have

$$
\frac{1}{2} \int_{\mathbb{R}^{3}}\left(c_{2}^{2}\right)_{t} \mathrm{~d} x+\int_{\mathbb{R}^{3}}\left|\nabla c_{2}\right|^{2} \mathrm{~d} x+\left(a_{22}-n_{\infty} a_{21}\right) \int_{\mathbb{R}^{3}}\left|c_{2}\right|^{2} \mathrm{~d} x \leq a_{21}\|\rho\|_{H^{2}} \int_{\mathbb{R}^{3}}\left|c_{2}\right|^{2} \mathrm{~d} x .
$$

Consider the linear combination $d_{1} \times(3.24)+(3.25)+(3.26)+(3.27)$, where $d_{1}=\frac{p^{\prime}\left(n_{\infty}\right)}{n_{\infty}^{2}}$. We see that as long as $\mathcal{E}_{N}^{\frac{1}{2}}(U)=\|U\|_{H^{N}}$ is small so that

$$
\begin{aligned}
& \left(a_{12}-n_{\infty} a_{11}\right)>a_{11} \mathcal{E}_{N}^{\frac{1}{2}}(U), \\
& \left(a_{22}-n_{\infty} a_{21}\right)>a_{21} \mathcal{E}_{N}^{\frac{1}{2}}(U)
\end{aligned}
$$


are satisfied, the linear combination yields

$$
\begin{aligned}
& \frac{1}{2} \frac{d}{d t} \int_{\mathbb{R}^{3}}\left(d_{1}|\rho|^{2}+|u|^{2}+\left|c_{1}\right|^{2}+\left|c_{2}\right|^{2}\right) \mathrm{d} x+n_{\infty} \int_{\mathbb{R}^{3}}|\rho|^{2} \mathrm{~d} x+\delta \int_{\mathbb{R}^{3}}|\nabla u|^{2} \mathrm{~d} x \\
& +\int_{\mathbb{R}^{3}}\left|\nabla c_{1}\right|^{2} \mathrm{~d} x+\int_{\mathbb{R}^{3}}\left|\nabla c_{2}\right|^{2} \mathrm{~d} x+\left(a_{12}-n_{\infty} a_{11}\right) \int_{\mathbb{R}^{3}}\left|c_{1}\right|^{2} \mathrm{~d} x+\left(a_{22}-n_{\infty} a_{21}\right) \int_{\mathbb{R}^{3}}\left|c_{2}\right|^{2} \mathrm{~d} x \leq 0 .
\end{aligned}
$$

Now, we make estimates on the high-order derivatives of $\left(\rho, u, c_{1}, c_{2}\right)$. Take $\alpha$ with $1 \leq|\alpha| \leq$ $N$. Applying $\partial^{\alpha}$ to the first equation of (2.2), multiplying by $\partial^{\alpha} \rho$ and then integrating in $x$, we have

$$
\begin{aligned}
\int_{\mathbb{R}^{3}} \partial^{\alpha} \rho \partial^{\alpha} \rho_{t} \mathrm{~d} x & +n_{\infty} \int_{\mathbb{R}^{3}} \partial^{\alpha} \rho \partial^{\alpha} \nabla \cdot u \mathrm{~d} x+n_{\infty} \int_{\mathbb{R}^{3}} \partial^{\alpha} \rho \partial^{\alpha} \rho \mathrm{d} x \\
& =-\int_{\mathbb{R}^{3}} \partial^{\alpha} \rho \partial^{\alpha} \nabla \cdot(\rho u) \mathrm{d} x-\int_{\mathbb{R}^{3}} \partial^{\alpha} \rho \partial^{\alpha} \rho^{2} \mathrm{~d} x
\end{aligned}
$$

By using integration by parts and Cauchy-Schwarz inequality, we obtain

$$
\begin{gathered}
\frac{1}{2} \frac{d}{d t} \int_{\mathbb{R}^{3}}\left(\partial^{\alpha} \rho\right)^{2} \mathrm{~d} x+n_{\infty} \int_{\mathbb{R}^{3}}\left|\partial^{\alpha} \rho\right|^{2} \mathrm{~d} x+n_{\infty} \int_{\mathbb{R}^{3}} \partial^{\alpha} \rho \partial^{\alpha} \nabla \cdot u \mathrm{~d} x \\
\quad=\int_{\mathbb{R}^{3}} \partial^{\alpha} \rho \sum_{\beta=0}^{\alpha} C_{\alpha}^{\beta} \partial^{\beta} \nabla \cdot u \partial^{\alpha-\beta} \rho \mathrm{d} x+\int_{\mathbb{R}^{3}} \partial^{\alpha} \rho \sum_{\beta=0}^{\alpha} C_{\alpha}^{\beta} \partial^{\beta} u \partial^{\alpha-\beta} \nabla \rho \mathrm{d} x-\int_{\mathbb{R}^{3}} \partial^{\alpha} \rho \partial^{\alpha} \rho^{2} \mathrm{~d} x \\
\leq C\|u\|_{H^{N}} \int_{\mathbb{R}^{3}}\left|\partial^{\alpha} \rho\right|^{2}+C\|\rho\|_{H^{N}} \int_{\mathbb{R}^{3}}\left|\partial^{\alpha} \rho\right|^{2}+\left|\partial^{\alpha} \nabla u\right|^{2} \mathrm{~d} x .
\end{gathered}
$$

Similarly for $\partial^{\alpha} u$, what follows from $(2.2)_{2}$ is

$$
\begin{aligned}
\frac{1}{2} \frac{d}{d t} \int_{\mathbb{R}^{3}}\left(\partial^{\alpha} u\right)^{2} \mathrm{~d} x & -\delta \int_{\mathbb{R}^{3}} \partial^{\alpha} u \partial^{\alpha} \Delta u \mathrm{~d} x+\frac{p^{\prime}\left(n_{\infty}\right)}{n_{\infty}} \int_{\mathbb{R}^{3}} \partial^{\alpha} u \partial^{\alpha} \nabla \rho \mathrm{d} x \\
& =-\int_{\mathbb{R}^{3}} \partial^{\alpha} u \partial^{\alpha}(u \cdot \nabla u) \mathrm{d} x+\int_{\mathbb{R}^{3}} \partial^{\alpha} u \partial^{\alpha} \nabla c_{1}^{2} \mathrm{~d} x-\int_{\mathbb{R}^{3}} \partial^{\alpha} u \partial^{\alpha} \nabla c_{2}^{2} \mathrm{~d} x \\
& \left.-\int_{\mathbb{R}^{3}} \partial^{\alpha} u \partial^{\alpha}\left(\frac{p^{\prime}\left(\rho+n_{\infty}\right)}{\rho+n_{\infty}}-\frac{p^{\prime}\left(n_{\infty}\right)}{n_{\infty}}\right) \nabla \rho\right) \mathrm{d} x .
\end{aligned}
$$

By using integration by parts and the Cauchy-Schwarz inequality, we have

$$
\begin{aligned}
& \frac{1}{2} \frac{d}{d t} \int_{\mathbb{R}^{3}}\left(\partial^{\alpha} u\right)^{2} \mathrm{~d} x+\delta \int_{\mathbb{R}^{3}}\left|\partial^{\alpha} \nabla u\right|^{2} \mathrm{~d} x-\frac{p^{\prime}\left(n_{\infty}\right)}{n_{\infty}} \int_{\mathbb{R}^{3}} \partial^{\alpha} \nabla \cdot u \partial^{\alpha} \rho \mathrm{d} x \\
& \leq C\|u\|_{H^{N}} \int_{\mathbb{R}^{3}}\left|\partial^{\alpha} u\right|^{2} \mathrm{~d} x+C\left\|c_{1}\right\|_{H^{N}} \int_{\mathbb{R}^{3}}\left(\left|\partial^{\alpha} u\right|^{2}+\left|\partial^{\alpha} \nabla c_{1}\right|^{2}\right) \mathrm{d} x \\
& \quad+C\left\|c_{2}\right\|_{H^{N}} \int_{\mathbb{R}^{3}}\left(\left|\partial^{\alpha} u\right|^{2}+\left|\partial^{\alpha} \nabla c_{2}\right|^{2}\right) \mathrm{d} x+C\|\rho\|_{H^{N}} \int_{\mathbb{R}^{3}}\left|\partial^{\alpha} u\right|^{2} \mathrm{~d} x+\left|\partial^{\alpha} \rho\right|^{2} \mathrm{~d} x .
\end{aligned}
$$


Similarly, we estimate $c_{1}, c_{2}$ as follows:

$$
\begin{aligned}
& \frac{1}{2} \frac{d}{d t} \int_{\mathbb{R}^{3}}\left(\partial^{\alpha} c_{1}\right)^{2}+\int_{\mathbb{R}^{3}}\left|\nabla \partial^{\alpha} c_{1}\right|^{2} \mathrm{~d} s+\left(a_{12}-n_{\infty} a_{11}\right) \int_{\mathbb{R}^{3}}\left|\partial^{\alpha} c_{1}\right|^{2} \mathrm{~d} s \\
& \leq C\|\rho\|_{H^{N}} \int_{\mathbb{R}^{3}}\left\|\left.\partial^{\alpha} c_{1}\right|^{2} \mathrm{~d} s+C\right\| c_{1} \|_{H^{N}} \int_{\mathbb{R}^{3}}\left(\left|\partial^{\alpha} c_{1}\right|^{2}+\left|\partial^{\alpha} \rho\right|^{2}\right) \mathrm{d} s
\end{aligned}
$$

and

$$
\begin{aligned}
& \frac{1}{2} \frac{d}{d t} \int_{\mathbb{R}^{3}}\left(\partial^{\alpha} c_{2}\right)^{2}+\int_{\mathbb{R}^{3}}\left|\nabla \partial^{\alpha} c_{2}\right|^{2} \mathrm{~d} s+\left(a_{22}-n_{\infty} a_{21}\right) \int_{\mathbb{R}^{3}}\left|\partial^{\alpha} c_{2}\right|^{2} \mathrm{~d} s \\
& \quad \leq C\|\rho\|_{H^{N}} \int_{\mathbb{R}^{3}}\left\|\left.\partial^{\alpha} c_{2}\right|^{2} \mathrm{~d} s+C\right\| c_{2} \|_{H^{N}} \int_{\mathbb{R}^{3}}\left(\left|\partial^{\alpha} c_{2}\right|^{2}+\left|\partial^{\alpha} \rho\right|^{2}\right) \mathrm{d} s
\end{aligned}
$$

Then, after taking the summation over $1 \leq|\alpha| \leqslant N$ and the combination $(3.29) \times d_{1}+$ $(3.30)+(3.31)+(3.32)$, we obtain

$$
\begin{aligned}
\frac{1}{2} \frac{d}{d t} \sum_{1 \leq|\alpha| \leq N} C_{\alpha} \int_{\mathbb{R}^{3}}\left|\partial^{\alpha}\left(\rho, u, c_{1}, c_{2}\right)\right|^{2} & \lambda_{1} \sum_{1 \leq|\alpha| \leq N} \int_{\mathbb{R}^{3}}\left|\partial^{\alpha} \nabla\left(u, c_{1}, c_{2}\right)\right|^{2} \mathrm{~d} x \\
& +\lambda_{2} \sum_{1 \leq|\alpha| \leq N} \int_{\mathbb{R}^{3}}\left|\partial^{\alpha}\left(\rho, c_{1}, c_{2}\right)\right|^{2} \mathrm{~d} x \leq 0,
\end{aligned}
$$

for some positive constants $C_{\alpha}, \lambda_{1}$ and $\lambda_{2}$. Therefore (3.23) follows from the further linear combination of (3.28) and (3.33) and the time integration over $[0, T]$. This completes the proof of Lemma 3.0.2.

Now, we are ready to present the proof of Proposition 2.0.1.

Proof of Proposition 2.0.1. Choose a positive constant $M=\min \left\{\epsilon_{0}, \epsilon_{1}\right\}$, where $\epsilon_{0}>0$ and $\epsilon_{1}>0$ are given in Lemma 3.0.1 and Lemma 3.0.2.

Let $U_{0} \in H^{N}\left(\mathbb{R}^{3}\right)$ satisfy $\left\|U_{0}\right\|_{H^{N}}<\frac{M}{2 \sqrt{C_{0}+1}}$. Now, let us define

$$
T=\left\{t \geq 0: \sup _{0 \leq s \leq t}\|U(s)\|_{H^{N}} \leq M\right\}
$$

Since $\left\|U_{0}\right\|_{H^{N}} \leq \frac{M}{2 \sqrt{C_{0}+1}} \leq \frac{M}{2}<M \leq \epsilon_{0}$, then $T>0$ holds from the local existence result. If $T$ is finite, from the definition of $T$, we have

$$
\sup _{0 \leq s \leq t}\|U\|_{H^{N}}=M .
$$


On the other hand, from a priori estimates, we have

$$
\sup _{0 \leq s \leq t}\|U(s)\|_{H^{N}} \leq \sqrt{C_{0}}\left\|U_{0}\right\|_{H^{N}} \leq \frac{M \sqrt{C_{0}}}{2 \sqrt{C_{0}+1}} \leq \frac{M}{2},
$$

which is a contradiction to (3.34). Therefore, $T=\infty$ holds. This implies that the local solution $U(t)$ obtained in Lemma 3.0.1 can be extended to infinity in time. Thus, we have a global solution $\left(\rho, u, c_{1}, c_{2}\right)(t) \in C\left([0, \infty) ; H^{N}\right)$. This completes the proof of Proposition 2.0.1. 


\section{Chapter 4}

\section{Linearized homogeneous system}

In this chapter, to study the time-decay property of solutions to the nonlinear system (2.2), we have to consider the following Cauchy problem arising from the system (2.2)-(2.3)

$$
\left\{\begin{array}{l}
\partial_{t} \rho+n_{\infty} \nabla \cdot u+n_{\infty} \rho=g_{1} \\
\partial_{t} u-\delta \Delta u+\frac{p^{\prime}\left(n_{\infty}\right)}{n_{\infty}} \nabla \rho=g_{2} \\
\partial_{t} c_{1}-\Delta c_{1}+\left(a_{12}-a_{11}\right) c_{1}=g_{3} \\
\partial_{t} c_{2}-\Delta c_{2}+\left(a_{22}-a_{21}\right) c_{2}=g_{4},
\end{array}\right.
$$

with initial data

$$
\left.\left(\rho, u, c_{1}, c_{2}\right)\right|_{t=0}=U_{0}=\left(\rho_{0}, u_{0}, c_{1,0}, c_{2,0}\right) .
$$

Here, the nonlinear source term takes the form

$$
\left\{\begin{array}{l}
g_{1}=-\nabla \cdot(\rho u)-\rho^{2} \\
g_{2}=-u \cdot \nabla u+\nabla c_{1}^{2}-\nabla c_{2}^{2}-\left(\frac{p^{\prime}\left(\rho+n_{\infty}\right)}{\rho+n_{\infty}}-\frac{p^{\prime}\left(n_{\infty}\right)}{n_{\infty}}\right) \nabla \rho . \\
g_{3}=a_{11} \rho c_{1} \\
g_{4}=a_{21} \rho c_{2} .
\end{array}\right.
$$

To obtain the time-decay rates of the solution to the system (4.1) in the next chapter, we are concerned with the following Cauchy problem for the linearized homogenous system corresponding to $(4.1)$ 


$$
\left\{\begin{array}{l}
\partial_{t} \rho+n_{\infty} \nabla \cdot u+n_{\infty} \rho=0 \\
\partial_{t} u-\delta \Delta u+\frac{p^{\prime}\left(n_{\infty}\right)}{n_{\infty}} \nabla \rho=0 \\
\partial_{t} c_{1}-\Delta c_{1}+\left(a_{12}-a_{11}\right) c_{1}=0 \\
\partial_{t} c_{2}-\Delta c_{2}+\left(a_{22}-a_{21}\right) c_{2}=0
\end{array}\right.
$$

In this chapter, we always denote $U_{1}=[\rho, u]$ as the solution to the linearized homogeneous system

$$
\left\{\begin{array}{l}
\partial_{t} \rho+n_{\infty} \nabla \cdot u+n_{\infty} \rho=0 \\
\partial_{t} u-\delta \Delta u+\frac{p^{\prime}\left(n_{\infty}\right)}{n_{\infty}} \nabla \rho=0,
\end{array}\right.
$$

with the initial data $\left.U_{1}\right|_{t=0}=U_{1,0}=\left(\rho_{0}, u_{0}\right)$ in $\mathbb{R}^{3}$.

\subsubsection{Representation of solutions}

We first find the explicit representation of the Fourier transform of the solution $U_{1}=[\rho, u]$ for the system

$$
\left\{\begin{array}{l}
\rho_{t}+n_{\infty} \nabla \cdot u+n_{\infty} \rho=0 \\
u_{t}-\delta \Delta u+\frac{p^{\prime}\left(n_{\infty}\right)}{n_{\infty}} \nabla \rho=0
\end{array}\right.
$$

with initial data $\left.U_{1}\right|_{t=0}=U_{1,0}=\left(\rho_{0}, u_{0}\right)$.

After taking the Fourier transform in $x$ for the first equation of (4.6), we have

$$
\hat{\rho}_{t}+n_{\infty} i \xi \hat{u}+n_{\infty} \hat{\rho}=0
$$

with initial data $\left.\hat{\rho}\right|_{t=0}=\hat{\rho}_{0}$.

Similarly, by taking the Fourier transform for the second equation of (4.6), we get

$$
\hat{u}_{t}+\delta|\xi|^{2} \hat{u}+\frac{p^{\prime}\left(n_{\infty}\right)}{n_{\infty}} i \xi \hat{\rho}=0
$$

with initial data $\left.\hat{u}\right|_{t=0}=\hat{u}_{0}$.

Further, by taking the dot product of (4.8) with $\hat{\xi}$, we have

$$
\tilde{\xi} \cdot \hat{u}_{t}+\delta|\xi|^{2} \tilde{\xi} \cdot \hat{u}+i \frac{p^{\prime}\left(n_{\infty}\right)}{n_{\infty}} \tilde{\xi} \cdot \xi \hat{\rho}=0
$$


Here and in the sequel we set $\tilde{\xi}=\frac{\xi}{|\xi|}$ for $|\xi| \neq 0$.

Then, we have

$$
\left\{\begin{array}{l}
\hat{\rho}_{t}+i n_{\infty} \xi \cdot \hat{u}+n_{\infty} \hat{\rho}=0 \\
\tilde{\xi} \cdot \hat{u}_{t}+\delta|\xi|^{2} \tilde{\xi} \cdot \hat{u}+i \frac{p^{\prime}\left(n_{\infty}\right)}{n_{\infty}} \tilde{\xi} \cdot \xi \hat{\rho}=0
\end{array}\right.
$$

We can rewrite (4.10) as

$$
\partial_{t} \hat{U}=A(\xi) \hat{U}
$$

with $\hat{U}(t, \xi)=(\hat{\rho}(t, \xi), \tilde{\xi} \cdot \hat{u}(t, \xi))^{T}$ and

$$
A(\xi)=\left[\begin{array}{cc}
-n_{\infty} & -i n_{\infty}|\xi| \\
-i \frac{p^{\prime}\left(n_{\infty}\right)}{n_{\infty}}|\xi| & -\delta|\xi|^{2}
\end{array}\right]
$$

where $T$ denotes the transpose of a row vector. Then,

$$
\operatorname{det}(A-\lambda I)=\lambda^{2}+\left(\delta \xi^{2}+n_{\infty}\right) \lambda+\delta n_{\infty}|\xi|^{2}+p^{\prime}\left(n_{\infty}\right)|\xi|^{2}=0 .
$$

The eigenvalues of the system are as follows

$$
\begin{aligned}
& \left.\lambda_{1}=-\frac{1}{2}\left(\delta \xi^{2}+n_{\infty}\right)+\frac{1}{2} \sqrt{\left(\delta \xi^{2}+n_{\infty}\right)^{2}-4|\xi|^{2}\left(\delta n_{\infty}+p^{\prime}\left(n_{\infty}\right)\right.}\right) \\
& \left.\lambda_{2}=-\frac{1}{2}\left(\delta \xi^{2}+n_{\infty}\right)-\frac{1}{2} \sqrt{\left(\delta \xi^{2}+n_{\infty}\right)^{2}-4|\xi|^{2}\left(\delta n_{\infty}+p^{\prime}\left(n_{\infty}\right)\right.}\right)
\end{aligned}
$$

Therefore, the eigenvectors corresponding to the eigenvalues $\lambda$ of $A(\xi)$ that satisfy $(A-$ $\lambda I) X=0$ are

$$
v_{1}=\left[\begin{array}{c}
i n_{\infty}|\xi| \\
-\left(n_{\infty}+\lambda_{1}\right)
\end{array}\right]
$$

and

$$
v_{2}=\left[\begin{array}{c}
i n_{\infty}|\xi| \\
-\left(n_{\infty}+\lambda_{2}\right)
\end{array}\right] \text {. }
$$

From the work above, one can define the general solution of (4.10) as

$$
\left[\begin{array}{c}
\hat{\rho} \\
\tilde{\xi} \cdot \hat{u}
\end{array}\right]=\left[\begin{array}{cc}
i n_{\infty}|\xi| e^{\lambda_{1} t} & i n_{\infty}|\xi| e^{\lambda_{2} t} \\
-\left(n_{\infty}+\lambda_{1}\right) e^{\lambda_{1} t} & -\left(n_{\infty}+\lambda_{2}\right) e^{\lambda_{2} t}
\end{array}\right]\left[\begin{array}{l}
d_{1} \\
d_{2}
\end{array}\right]
$$


where $d_{1}, d_{2}$ satisfy

$$
\left[\begin{array}{c}
\left.\hat{\rho}\right|_{t=0} \\
\left.\tilde{\xi} \cdot \hat{u}\right|_{t=0}
\end{array}\right]=\left[\begin{array}{cc}
\operatorname{in}_{\infty}|\xi| & \operatorname{in}_{\infty}|\xi| \\
-\left(n_{\infty}+\lambda_{1}\right) & -\left(n_{\infty}+\lambda_{2}\right)
\end{array}\right]\left[\begin{array}{l}
d_{1} \\
d_{2}
\end{array}\right]
$$

From this, we deduce that

$$
\left[\begin{array}{c}
d_{1} \\
d_{2}
\end{array}\right]=\frac{1}{i n_{\infty}|\xi|\left(\lambda_{1}-\lambda_{2}\right)}\left[\begin{array}{cc}
-\left(n_{\infty}+\lambda_{2}\right) & -i n_{\infty}|\xi| \\
\left(n_{\infty}+\lambda_{1}\right) & i n_{\infty}|\xi|
\end{array}\right]\left[\begin{array}{c}
\hat{\rho}_{0} \\
\tilde{\xi} \cdot \hat{u}_{0}
\end{array}\right]
$$

Therefore, we have

$$
\left[\begin{array}{c}
\hat{\rho} \\
\tilde{\xi} \cdot \hat{u}
\end{array}\right]=\frac{1}{i n_{\infty}|\xi|\left(\lambda_{1}-\lambda_{2}\right)}\left[\begin{array}{cc}
i n_{\infty}|\xi| e^{\lambda_{1} t} & i n_{\infty}|\xi| e^{\lambda_{2} t} \\
-\left(n_{\infty}+\lambda_{1}\right) e^{\lambda_{1} t} & -\left(n_{\infty}+\lambda_{2}\right) e^{\lambda_{2} t}
\end{array}\right]\left[\begin{array}{cc}
-\left(n_{\infty}+\lambda_{2}\right) & -i n_{\infty}|\xi| \\
\left(n_{\infty}+\lambda_{1}\right) & i n_{\infty}|\xi|
\end{array}\right]\left[\begin{array}{c}
\hat{\rho}_{0} \\
\tilde{\xi} \cdot \hat{u}_{0}
\end{array}\right] .
$$

It is straightforward to obtain

$$
\hat{\rho}=\frac{\left(\lambda_{1}+n_{\infty}\right) e^{\lambda_{2} t}-\left(\lambda_{2}+n_{\infty}\right) e^{\lambda_{1} t}}{\left(\lambda_{1}-\lambda_{2}\right)} \hat{\rho}_{0}-i n_{\infty} \frac{e^{\lambda_{1} t}-e^{\lambda_{2} t}}{\left(\lambda_{1}-\lambda_{2}\right)} \xi \cdot \hat{u_{0}}
$$

and

$$
\tilde{\xi} \cdot \hat{u}=\frac{\left(n_{\infty}+\lambda_{1}\right)\left(n_{\infty}+\lambda_{2}\right)}{i n_{\infty}|\xi|}\left(\frac{e^{\lambda_{1} t}-e^{\lambda_{2} t}}{\lambda_{1}-\lambda_{2}}\right) \hat{\rho}_{0}+\frac{\left(\lambda_{1}+n_{\infty}\right) e^{\lambda_{1} t}-\left(\lambda_{2}+n_{\infty}\right) e^{\lambda_{2} t}}{\lambda_{1}-\lambda_{2}} \tilde{\xi} \cdot \hat{u}_{0} .
$$

Moreover, by taking the curl for the second equation of (4.6), we have

$$
\nabla \times u_{t}-\delta \nabla \times \Delta u+\frac{p^{\prime}\left(n_{\infty}\right)}{n_{\infty}} \nabla \times \nabla \rho=0,
$$

since $\nabla \times \nabla \rho=0$ implies

$$
\partial_{t}(\nabla \times u)-\delta \nabla \times \Delta u=0 .
$$

Taking the Fourier transform in $x$ for the above equation, we have

$$
\partial_{t}(\tilde{\xi} \times \hat{u})+\delta|\xi|^{2}(\tilde{\xi} \times \hat{u})=0 .
$$

Initial data is given as

$$
\left.(\tilde{\xi} \times \hat{u})\right|_{t=0}=\tilde{\xi} \times \hat{u}_{0} .
$$

By solving the initial value problem (4.18) and (4.19), we have

$$
\tilde{\xi} \times \hat{u}=e^{-\delta|\xi|^{2} t} \tilde{\xi} \times \hat{u}_{0} .
$$


For $t \geq 0$ and $\xi \in \mathbb{R}^{3}$ with $|\xi| \neq 0$, one has the decomposition $\hat{u}=\tilde{\xi} \tilde{\xi} \cdot \hat{u}-\tilde{\xi} \times(\tilde{\xi} \times \hat{u})$. It is straightforward to get

$$
\begin{aligned}
\hat{u}= & \frac{\left(n_{\infty}+\lambda_{1}\right)\left(n_{\infty}+\lambda_{2}\right)}{i n_{\infty}|\xi|^{2}}\left(\frac{e^{\lambda_{1} t}-e^{\lambda_{2} t}}{\lambda_{1}-\lambda_{2}}\right) \xi \cdot \hat{\rho}_{0} \\
& +\left(\frac{\left(\lambda_{1}+n_{\infty}\right) e^{\lambda_{1} t}-\left(\lambda_{2}+n_{\infty}\right) e^{\lambda_{2} t}}{\lambda_{1}-\lambda_{2}}\right) \tilde{\xi} \tilde{\xi} \cdot \hat{u}_{0}-e^{-\delta|\xi|^{2} t} \tilde{\xi} \times\left(\tilde{\xi} \times \hat{u}_{0}\right) .
\end{aligned}
$$

Then

$$
\begin{aligned}
\hat{u}= & \frac{\left(n_{\infty}+\lambda_{1}\right)\left(n_{\infty}+\lambda_{2}\right)}{i n_{\infty}|\xi|}\left(\frac{e^{\lambda_{1} t}-e^{\lambda_{2} t}}{\lambda_{1}-\lambda_{2}}\right) \frac{\xi}{|\xi|} \hat{\rho}_{0} \\
& +\left(\frac{\left(\lambda_{1}+n_{\infty}\right) e^{\lambda_{1} t}-\left(\lambda_{2}+n_{\infty}\right) e^{\lambda_{2} t}}{\lambda_{1}-\lambda_{2}}\right) \frac{\xi \otimes \xi}{|\xi|^{2}} \hat{u}_{0}+e^{-\delta|\xi|^{2} t}\left(I_{3}-\frac{\xi \otimes \xi}{|\xi|^{2}}\right) \hat{u}_{0}
\end{aligned}
$$

After summarizing the above computations on the explicit representation of the Fourier transform of the solution $U_{1}=[\rho, u]$, we have

$$
\left[\begin{array}{l}
\hat{\rho}(t, \xi) \\
\hat{u}(t, \xi)
\end{array}\right]=\hat{G}(t, \xi)\left[\begin{array}{l}
\hat{\rho}(0, \xi) \\
\hat{u}(0, \xi)
\end{array}\right] .
$$

We can verify the exact expression of the Fourier transform $\hat{G}(t, \xi)$ of Green's function $G(t, \xi)=e^{t B}$ as

$$
\begin{aligned}
\hat{G}(t, \xi) & =\left[\begin{array}{ll}
\hat{G}_{11} & \hat{G}_{12} \\
\hat{G}_{21} & \hat{G}_{22}
\end{array}\right] \\
& =\left[\begin{array}{cc}
\frac{\left(\lambda_{1}+n_{\infty}\right) e^{\lambda_{2} t}-\left(\lambda_{2}+n_{\infty}\right) e^{\lambda_{1} t}}{\lambda_{1}-\lambda_{2}} & -i n_{\infty} \xi \frac{e^{\lambda_{1} t}-e^{\lambda_{2} t}}{\left(\lambda_{1}-\lambda_{2}\right)} \\
\frac{\left(n_{\infty}+\lambda_{1}\right)\left(n_{\infty}+\lambda_{2}\right) \xi}{i n_{\infty}|\xi|^{2}}\left(\frac{e^{\lambda_{1} t}-e^{\lambda_{2} t}}{\lambda_{1}-\lambda_{2}}\right) & \frac{\left(\lambda_{1}+n_{\infty}\right) e^{\lambda_{1} t}-\left(\lambda_{2}+n_{\infty}\right) e^{\lambda_{2} t}}{\lambda_{1}-\lambda_{2}} \frac{\xi \otimes \xi}{|\xi|^{2}}+e^{-\delta \xi^{2} t}\left(I_{3}-\frac{\xi \otimes \xi}{|\xi|^{2}}\right)
\end{array}\right] .
\end{aligned}
$$

\subsection{2 $L^{2}-L^{q}$ time-decay property}

In this subsection, we use (4.23) to obtain the refined $L^{2}-L^{q}$ time decay property for

$$
U_{1}=(\rho, u)=e^{t B} U_{1,0}
$$

where $e^{t B}$ is the linear solution operator for $t \geq 0$. For this, we need to find the timefrequency pointwise estimate on $\hat{\rho}, \hat{u}$ in the following Lemma: 
Lemma 4.0.1. Let $U_{1}=[\rho, u]$ be the solution to the linear homogeneous system (4.6) with the initial data $\left.U_{1}\right|_{t=0}=\left(\rho_{0}, u_{0}\right)$. Then there exist constants $\epsilon>0, \lambda>0, C>0$ such that for all $t>0,|\xi| \leq \epsilon$,

$$
\begin{gathered}
|\hat{\rho}(t, \xi)| \leq C\left(|\xi|^{2} e^{-\lambda|\xi|^{2} t}+e^{-n_{\infty} \lambda t}\right)\left|\hat{\rho}_{0}(\xi)\right|+C\left(|\xi| e^{-\lambda|\xi|^{2} t}+|\xi| e^{-n_{\infty} \lambda t}\right)\left|\hat{u}_{0}(\xi)\right| \\
|\hat{u}(t, \xi)| \leq C|\xi|\left(e^{-\lambda|\xi|^{2} t}+e^{-n_{\infty} \lambda t}\right)\left|\hat{\rho}_{0}(\xi)\right|+C\left(e^{-\lambda|\xi|^{2} t}+|\xi|^{2} e^{-n_{\infty} \lambda t}\right)\left|\hat{u}_{0}(\xi)\right|
\end{gathered}
$$

and for all $t>0,|\xi| \geq \epsilon$,

$$
\begin{aligned}
|\hat{\rho}(t, \xi)| & \leq C e^{-\lambda t}\left|\hat{\rho}_{0}(\xi), \hat{u}_{0}(\xi)\right|, \\
|\hat{u}(t, \xi)| & \leq C e^{-\lambda t}\left|\hat{\rho}_{0}(\xi), \hat{u}_{0}(\xi)\right| .
\end{aligned}
$$

Proof. In order to obtain the upper bound of $\hat{\rho}(t, \xi)$ and $\hat{u}(t, \xi)$, we have to estimate $\hat{G}_{11}, \hat{G}_{12}, \hat{G}_{21}$, and $\hat{G}_{22}$ in (4.23). To do so, we need to deal with the low frequency $|\xi| \leq \epsilon$ and high frequency $|\xi|>\epsilon$. By using the definition of the eigenvalue, we can analyze the eigenvalue for $|\xi| \rightarrow 0$ as

$$
\begin{array}{r}
\lambda_{1} \sim-O(1)|\xi|^{2}, \\
\lambda_{2} \sim-n_{\infty}+O(1)|\xi|^{2} .
\end{array}
$$

On the other hand, we have the leading orders of the eigenvalue for $|\xi| \rightarrow \infty$ as

$$
\begin{array}{r}
\lambda_{1} \sim-O(1), \\
\lambda_{2} \sim-\delta \xi^{2}+O(1) .
\end{array}
$$

Now, we can estimate $\hat{G}(t, \xi)$ as follows:

$$
\begin{aligned}
\left|\hat{G}_{11}\right| & \leq C\left(|\xi|^{2} e^{-\lambda|\xi|^{2} t}+e^{-n_{\infty} \lambda t}\right), \\
\left|\hat{G}_{12}\right| & \leq|\xi|\left(e^{-\lambda|\xi|^{2} t}+e^{-n_{\infty} \lambda t}\right), \\
\left|\hat{G}_{21}\right| & \leq C|\xi|\left(e^{-\lambda|\xi|^{2} t}+e^{-n_{\infty} \lambda t}\right), \\
\left|\hat{G}_{22}\right| & \leq C\left(e^{-\lambda|\xi|^{2} t}+|\xi|^{2} e^{-n_{\infty} \lambda t}\right)+C e^{-\delta|\xi|^{2} t}, \\
& \leq C\left(e^{-\lambda|\xi|^{2} t}+|\xi|^{2} e^{-n_{\infty} \lambda t}\right),
\end{aligned}
$$


as $|\xi| \leq \epsilon$, and

$$
\begin{aligned}
& \quad\left|\hat{G}_{11}\right| \leq C e^{-O(1) \lambda t} \leq C e^{-\lambda t}, \\
& \quad\left|\hat{G}_{12}\right|=\left|\hat{G}_{21}\right| \leq C e^{-\lambda t} \\
& \quad\left|\hat{G}_{22}\right| \leq C e^{-\delta|\xi|^{2} t}+C e^{-O(1) t} \leq C e^{-\lambda t},
\end{aligned}
$$

$\operatorname{as}|\xi|>\epsilon$.

When the eigenvalues coalesce we get decays exponentially:

$$
t \lambda_{1} e^{\lambda_{1} t} \leq e^{\frac{\lambda_{1}}{2} t} \leq C e^{-\lambda t}
$$

for $\frac{\lambda_{1}}{2} \leq-\lambda$.

Therefore, after plugging the above computations into (4.15) and (4.22), it holds that

$$
|\hat{\rho}(t, \xi)| \leq C\left(|\xi|^{2} e^{-\lambda|\xi|^{2} t}+e^{-n_{\infty} \lambda t}\right)\left|\hat{\rho}_{0}(\xi)\right|+C\left(|\xi| e^{-\lambda|\xi|^{2} t}+|\xi| e^{-n_{\infty} \lambda t}\right)\left|\hat{u}_{0}(\xi)\right|
$$

and

$$
|\hat{u}(t, \xi)| \leq C|\xi|\left(e^{-\lambda|\xi|^{2} t}+e^{-n_{\infty} \lambda t}\right)\left|\hat{\rho}_{0}(\xi)\right|+C\left(e^{-\lambda|\xi|^{2} t}+|\xi|^{2} e^{-n_{\infty} \lambda t}\right)\left|\hat{u}_{0}(\xi)\right|
$$

for $|\xi| \leq \epsilon$. This proves (4.24) and (4.25). Finally, (4.26) and (4.27) can be proven in the completely same way as for (4.24) and (4.25). This completes the proof of Lemma 4.0.1.

Theorem 4.0.2. Let $2 \leq q \leq \infty$, and let $m \geq 0$ be an integer. Suppose that $U_{1}=e^{B t} U_{1,0}$ is the solution to the Cauchy problem (4.6) with the initial data $U_{1,0}=\left(\rho_{0}, u_{0}\right)$. Then $U_{1}=[\rho, u]$ satisfies the following time-decay property:

$$
\begin{gathered}
\left\|\nabla^{m} \rho(t)\right\|_{L^{q}} \leq C(1+t)^{-\frac{3}{2}\left(1-\frac{1}{q}\right)-\frac{m+1}{2}}\left\|\rho_{0}, u_{0}\right\|_{L^{1}}+e^{-\lambda t}\left\|\nabla^{m+\left[3\left(\frac{1}{2}-\frac{1}{q}\right)\right]_{+}}\left(\rho_{0}, u_{0}\right)\right\|_{L^{2}}, \\
\left\|\nabla^{m} u(t)\right\|_{L^{q}} \leq C(1+t)^{-\frac{3}{2}\left(1-\frac{1}{q}\right)-\frac{m}{2}}\left\|\rho_{0}, u_{0}\right\|_{L^{1}}+e^{-\lambda t}\left\|\nabla^{m+\left[3\left(\frac{1}{2}-\frac{1}{q}\right)\right]_{+}}\left(\rho_{0}, u_{0}\right)\right\|_{L^{2}},
\end{gathered}
$$

for any $t \geq 0$, where $C=C(m, q)$ and $\left[3\left(\frac{1}{2}-\frac{1}{q}\right)\right]_{+}$is defined as

$$
\left[3\left(\frac{1}{2}-\frac{1}{q}\right)\right]_{+}=\left\{\begin{array}{l}
0 \quad \text { if } q=2 \\
{\left[3\left(\frac{1}{2}-\frac{1}{q}\right)\right]_{-}+1 \quad \text { if } q \neq 2}
\end{array}\right.
$$

where [.]_ denotes the integer part of the argument. 
Proof. Take $2 \leq q \leq \infty$ and an integer $m \geq 0$. Set $U_{1}=e^{B t} U_{1,0}$. From the Hausdorff-Young inequality,

$$
\left\|\nabla^{m} \rho(t)\right\|_{L^{q}\left(\mathbb{R}_{x}^{3}\right)} \leq C\left\||\xi|^{m} \hat{\rho}(t, \xi)\right\|_{L^{q^{\prime}\left(\mathbb{R}_{\xi}^{3}\right)}} \leq C\left\||\xi|^{m} \hat{\rho}(t, \xi)\right\|_{L^{q^{\prime}}(|\xi| \leq \epsilon)}+C\left\||\xi|^{m} \hat{\rho}(t, \xi)\right\|_{L^{q^{\prime}(|\xi| \geq \epsilon)}}
$$

where $\frac{1}{q}+\frac{1}{q^{\prime}}=1$.

We estimate the first term of (4.31) by using (4.24), as follows:

$$
\begin{aligned}
\||\xi|^{m} \hat{\rho}(t, \xi) & \|_{L^{q^{\prime}}(|\xi| \leq \epsilon)}^{q^{\prime}} \leq c \int_{|\xi| \leq \epsilon}\left[\left(|\xi|^{(m+2) q^{\prime}} e^{-\lambda q^{\prime}|\xi|^{2} t}+|\xi|^{m q^{\prime}} e^{-n_{\infty} \lambda q^{\prime} t}\right)\left|\hat{\rho}_{0}(\xi)\right|^{q^{\prime}}\right. \\
& \left.+c\left(|\xi|^{m q^{\prime}+q^{\prime}} e^{-\lambda q^{\prime}|\xi|^{2} t}+|\xi|^{m q^{\prime}+q^{\prime}} e^{-n_{\infty} \lambda q^{\prime} t}\right)\left|\hat{u}_{0}(\xi)\right|^{q \prime}\right] d \xi \\
& \leq C \sup _{\xi}\left|\hat{\rho}_{0}\right|^{q \prime} \int_{|\xi| \leq \epsilon}\left(|\xi|^{(m+2) q^{\prime}} e^{-q^{\prime} \lambda|\xi|^{2}(1+t)+q^{\prime} \lambda|\xi|^{2}}+|\xi|^{m q^{\prime}} e^{-n_{\infty} \lambda q^{\prime} t}\right) d \xi \\
& +C \sup _{\hat{\xi}}\left|\hat{u}_{0}\right|^{q \prime} \int_{|\xi| \leq \epsilon}\left(|\xi|^{(m+1) q^{\prime}} e^{-\lambda q^{\prime}|\xi|^{2}(1+t)+\lambda q^{\prime}|\xi|^{2}}+|\xi|^{(m+1) q^{\prime}} e^{-n_{\infty} \lambda q^{\prime} t}\right) d \xi \\
& \leq C(1+t)^{-\frac{m q^{\prime}+2 q^{\prime}+3}{2}}\left\|\rho_{0}\right\|_{L^{1}}^{q^{\prime}}+C(1+t)^{-\frac{m q^{\prime}+q^{\prime}+3}{2}}\left\|u_{0}\right\|_{L^{1}}^{q^{\prime}} \\
& +C e^{-n_{\infty} \lambda q^{\prime} t}\left\|\left[\rho_{0}, u_{0}\right]\right\|_{L^{1}}^{q^{\prime}}
\end{aligned}
$$

Thus,

$$
\begin{aligned}
\left\||\xi|^{m} \hat{\rho}(t, \xi)\right\|_{L^{q^{\prime}}(|\xi| \leq \epsilon)} & \leq C(1+t)^{-\frac{3}{2 q^{\prime}}-\frac{m+2}{2}}\left\|\rho_{0}\right\|_{L^{1}}+C(1+t)^{-\frac{3}{2 q^{\prime}}-\left(\frac{m+1}{2}\right)}\left\|u_{0}\right\|_{L^{1}} \\
& +C e^{-n_{\infty} \lambda t}\left\|\left[\rho_{0}, u_{0}\right]\right\|_{L^{1}} \\
& \leq C(1+t)^{-\frac{3}{2}\left[1-\frac{1}{q}\right]-\frac{m+1}{2}}\left\|\left[\rho_{0}, u_{0}\right]\right\|_{L^{1}}
\end{aligned}
$$

Now, we estimate the second term of (4.31) from (4.26) as

$$
\left\||\xi|^{m} \hat{\rho}(\xi, t)\right\|_{L^{q^{\prime}}(|\xi| \geq \epsilon)} \leq C\left[\int_{|\xi| \geq \epsilon}|\xi|^{m q^{\prime}} e^{-q^{\prime} \lambda t}\left|\hat{\rho}_{0}(\xi), \hat{u}_{0}(\xi)\right|^{q^{\prime}} d \xi\right]^{\frac{1}{q^{\prime}}}
$$


Now, take $\epsilon_{1}>0$ which is small enough. By the Hölder inequality $\frac{1}{q^{\prime}}=\frac{1}{2}+\frac{2-q^{\prime}}{2 q^{\prime}}$, we have

$$
\begin{aligned}
& \left\||\xi|^{m} \hat{\rho}(\xi, t)\right\|_{L^{q^{\prime}}(|\xi| \geq \epsilon)} \leq C\left[\int_{|\xi| \geq \epsilon}|\xi|^{-(3+\epsilon)\left(\frac{2-q^{\prime}}{2}\right)}|\xi|^{(3+\epsilon)\left(\frac{2-q^{\prime}}{2}\right)+m q^{\prime}} e^{-q^{\prime} \lambda t}\left|\hat{\rho}_{0}(\xi), \hat{u}_{0}(\xi)\right|^{q \prime} d \xi\right]^{\frac{1}{q^{\prime}}} \\
& \leq C e^{-\lambda t}\left[\int_{|\xi| \geq \epsilon}|\xi|^{-(3+\epsilon)} d \xi\right]^{\frac{2-q^{\prime}}{2 q^{\prime}}}\left[\int_{|\xi| \geq \epsilon}|\xi|^{\left((3+\epsilon)\left(\frac{2-q^{\prime}}{2}\right)+m q^{\prime}\right) \frac{2}{q^{\prime}}}\left|\hat{\rho}_{0}(\xi), \hat{u}_{0}(\xi)\right|^{q^{\prime}\left(\frac{2}{q^{\prime}}\right)} d \xi\right]^{\left(\frac{1}{q^{\prime}}\right)\left(\frac{q^{\prime}}{2}\right)} \\
& \leq\left. C e^{-\lambda t}\left\||\xi|^{-(3+\epsilon)}\right\|\right|^{\frac{2-q^{\prime}}{2 q^{\prime}}}\left\||\xi|^{(3+\epsilon) \frac{2-q^{\prime}}{2 q^{\prime}}+m}\left[\hat{\rho}_{0}(\xi), \hat{u}_{0}(\xi)\right]\right\|_{L^{2}} \\
& \leq C e^{-\lambda t}\left\|\nabla^{m+(3+\epsilon) \frac{2-q^{\prime}}{2 q^{\prime}}}\left[\rho_{0}, u_{0}\right]\right\|_{L^{2}} \\
& \leq C e^{-\lambda t}\left\|\nabla^{m+3\left[\frac{1}{q^{\prime}}-\frac{1}{2}\right]_{-}}\left[\rho_{0}, u_{0}\right]\right\|_{L^{2}} \\
& \leq C e^{-\lambda t}\left\|\nabla^{m+3\left[\frac{1}{2}-\frac{1}{q}\right]_{-}}\left[\rho_{0}, u_{0}\right]\right\|_{L^{2}}
\end{aligned}
$$

after plugging (4.33) and (4.32) into (4.31) implies (4.28).

To prove (4.29), it similarly holds that

$$
\left\|\nabla^{m} u(t)\right\|_{L^{q}\left(\mathbb{R}_{x}^{3}\right)} \leq C\left\|\left.|| \xi\right|^{m} \hat{u}(\xi, t)\right\|_{L^{q^{\prime}\left(\mathbb{R}_{\xi}^{3}\right)}} \leq C\left\||\xi|^{m} \hat{u}(\xi, t)\right\|_{L^{q^{\prime}(|\xi| \leq \epsilon)}}+C\left\|\left.|| \xi\right|^{m} \hat{u}(\xi, t)\right\|_{L^{q^{\prime}(|\xi| \geq \epsilon)}}
$$

where from (4.25), the first term is

$$
\begin{aligned}
\left\||\xi|^{m} \hat{u}(t, \xi)\right\|_{L^{q^{\prime}(|\xi| \leq \epsilon)}}^{q^{\prime}} & \leq C \int_{|\xi| \leq \epsilon}\left(|\xi|^{m q^{\prime}+q^{\prime}}\left(e^{-q^{\prime} \lambda|\xi|^{2}(t+1)}+e^{-n_{\infty} \lambda q^{\prime} t}\right)\left|\hat{\rho}_{0}(\xi)\right|^{q \prime}\right) d \xi \\
& +\left.C \int_{\xi \leq \epsilon}\left(|\xi|^{m q^{\prime}} e^{-\lambda q^{\prime}|\xi|^{2}(t+1)}+|\xi|^{(m+2) q^{\prime}} e^{-n_{\infty} \lambda q^{\prime} t}\right)\left|\hat{u}_{0}(\xi)\right|\right|^{\prime \prime} d \xi \\
& \leq C(1+t)^{-\frac{m q^{\prime}+q^{\prime}+3}{2}} \|\left[\rho_{0}\left\|_{L^{1}}^{q^{\prime}}+(1+t)^{-\frac{m q^{\prime}+3}{2}}\right\| u_{0} \|_{L^{1}}^{q^{\prime}}\right. \\
& +C e^{-n_{\infty} \lambda q^{\prime} t}\left\|\left[\rho_{0}, u_{0}\right]\right\|^{q^{\prime}} L^{1} .
\end{aligned}
$$

It follows that

$$
\begin{aligned}
\left\||\xi|^{m} \hat{u}(t, \xi)\right\|_{L^{q^{\prime}}(|\xi| \leq \epsilon)} & \leq C(1+t)^{-\frac{3}{2 q^{\prime}}-\frac{m+1}{2}} \|\left[\rho_{0} \|_{L^{1}}\right. \\
& +(1+t)^{-\frac{3}{2 q^{\prime}}-\frac{m}{2}}\left\|u_{0}\right\|_{L^{1}}+C e^{-n_{\infty} \lambda t}\left\|\left[\rho_{0}, u_{0}\right]\right\|_{L^{1}} \\
& \leq C(1+t)^{-\frac{3}{2}\left[1-\frac{1}{q}\right]-\frac{m+1}{2}} \|\left[\rho_{0}\left\|_{L^{1}}+(1+t)^{-\frac{3}{2}\left[1-\frac{1}{q}\right]-\frac{m}{2}}\right\| u_{0} \|_{L^{1}}\right. \\
& \leq C(1+t)^{-\frac{3}{2}\left[1-\frac{1}{q}\right]-\frac{m}{2}}\left\|\left[\rho_{0}, u_{0}\right]\right\|_{L^{1}} .
\end{aligned}
$$

Similar to obtaining (4.33), one has

$$
\left\||\xi|^{m} \hat{u}(\xi, t)\right\|_{L^{q^{\prime}}(|\xi| \geq \epsilon)} \leq C e^{-\lambda t}\left\|\nabla^{m+3\left[\frac{1}{2}-\frac{1}{q}\right]_{+}}\left[\rho_{0}, u_{0}\right]\right\|_{L^{2}} .
$$


Thus, plugging (4.35) and (4.36) into (4.34) implies (4.29). This completes the proof of Theorem 4.0.2.

Corollary 4.0.3. Assume that $U_{1}=e^{B t} U_{1,0}$ is the solution to the Cauchy problem (4.6) with initial data $U_{1,0}=\left[\rho_{0}, u_{0}\right]$. Then $U_{1}=[\rho, u]$ satisfies the following:

$$
\begin{aligned}
\|\rho(t)\|_{L^{2}} \leq C(1+t)^{-\frac{5}{4}}\left\|\left[\rho_{0}, u_{0}\right]\right\|_{L^{1}}+e^{-\lambda t}\left\|\left[\rho_{0}, u_{0}\right]\right\|_{L^{2}}, \\
\|u(t)\|_{L^{2}} \leq C(1+t)^{-\frac{3}{4}}\left\|\left[\rho_{0}, u_{0}\right]\right\|_{L^{1}}+e^{-\lambda t}\left\|\left[\rho_{0}, u_{0}\right]\right\|_{L^{2}}, \\
\|\rho(t)\|_{L^{\infty}} \leq C(1+t)^{-2}\left\|\left[\rho_{0}, u_{0}\right]\right\|_{L^{1}}+e^{-\lambda t}\left\|\left[\rho_{0}, u_{0}\right]\right\|_{\dot{H}^{2}}, \\
\|u(t)\|_{L^{\infty}} \leq C(1+t)^{-\frac{3}{2}}\left\|\left[\rho_{0}, u_{0}\right]\right\|_{L^{1}}+e^{-\lambda t}\left\|\left[\rho_{0}, u_{0}\right]\right\|_{\dot{H}^{2}} .
\end{aligned}
$$




\section{Chapter 5}

\section{Time-Decay Rates for the Nonlinear System}

In this chapter, we will prove (2.5)-(2.7) in Proposition 2.0.2. The main idea is to introduce a general approach to combine the energy estimates and spectral analysis. We will apply the linear $L^{2}-L^{q}$ time-decay property of the linearized homogeneous system (4.4), studied in the previous chapter, to the nonlinear case. We need the mild form of the original nonlinear Cauchy problem (2.2). Throughout this chapter, we suppose that $U=\left[\rho, u, c_{1}, c_{2}\right]$ is the solution to the Cauchy problem (2.3) with initial data $U_{0}=\left(\rho_{0}, u_{0}, c_{1,0}, c_{2,0}\right)$.

Then, by Duhamel's principle, the solution $U=\left[\rho, u, c_{1}, c_{2}\right]$ can be formally written as

$$
U(t)=e^{B t} U_{0}+\int_{0}^{t} e^{(t-s) B}\left[g_{1}, g_{2}, g_{3}, g_{4}\right] d s,
$$

where $e^{B t} U_{0}$ is the solution to the Cauchy problem (4.1) with initial data $U_{0}=\left(\rho_{0}, u_{0}, c_{1,0}, c_{2,0}\right)$. Here, the nonlinear source term takes the form (4.3).

\subsubsection{Time rate for the energy functional and high-order energy functional}

In this subsection, we will prove the time-decay rate for the energy functional $\|U(t)\|_{H^{N}}^{2}$ and the time-decay rate for the high-order energy functional $\|\nabla U(t)\|_{H^{N}}^{2}$. For that, we investigate the time-decay rates of solutions in Proposition 2.0.1 under extra conditions on the given 
initial data $U_{0}=\left[\rho_{0}, u_{0}, c_{1,0}, c_{2,0}\right]$. We define

$$
\epsilon_{H^{N}}\left(U_{0}\right)=\left\|U_{0}\right\|_{H^{N}}+\left\|\left[\rho_{0}, u_{0}\right]\right\|_{L^{1}},
$$

for an integer $N \geq 4$. We also define $\mathcal{E}_{N} U(t) \sim\left\|\left[\rho, u, c_{1}, c_{2}\right]\right\|_{H^{N}}^{2}$ as the energy functional and $\mathcal{D}_{N} U(t) \sim\left\|\left[\nabla\left(u, c_{1}, c_{2}\right)\right]\right\|_{H^{N}}^{2}, \mathcal{D}_{N}^{h} U(t) \sim\left\|\left[\rho, c_{1}, c_{2}\right]\right\|_{H^{N}}^{2}$ as the dissipation rates.

First, we start with this proposition for the energy functional and the high-order energy functional.

Proposition 5.0.1. Let $U=\left[\rho, u, c_{1}, c_{2}\right]$ be the solution to the Cauchy problem (2.2) with initial data $U_{0}=\left(\rho_{0}, u_{0}, c_{1,0}, c_{2,0}\right)$. If $\epsilon_{N+1}\left(U_{0}\right)>0$ is small enough, then the solution $U=\left[\rho, u, c_{1}, c_{2}\right]$ satisfies

$$
\|U(t)\|_{H^{N}} \leq \epsilon_{N+1}\left(U_{0}\right)(1+t)^{\frac{-3}{4}},
$$

and

$$
\|\nabla U(t)\|_{H^{N}} \leq \epsilon_{N+1}\left(U_{0}\right)(1+t)^{\frac{-5}{4}},
$$

for any $t \geq 0$.

Proof. Suppose $\epsilon_{N+1}\left(U_{0}\right)$ is sufficiently small. From Proposition 2.0.1 the solution $U=$ $\left[\rho, u, c_{1}, c_{2}\right]$ satisfies:

$$
\frac{d}{d t} \mathcal{E}_{N}(U(t))+\lambda_{1} \mathcal{D}_{N}(U(t))+\lambda_{2} \mathcal{D}_{N}^{h}(U(t)) \leq 0,
$$

for $t \geq 0$.

Now, we proceed by making the time-weighted estimate and iteration for the inequality (5.5). Let $l \geq 0$. Multiplying (5.5) by $(1+t)^{l}$ and integrating over $[0, t]$ gives

$$
\begin{aligned}
(1+t)^{l} \mathcal{E}_{N} U(t)+ & \lambda_{1} \int_{0}^{t}(1+s)^{l} \mathcal{D}_{N}(U(s)) d s+\lambda_{2} \int_{0}^{t}(1+s)^{l} \mathcal{D}_{N}^{h}(U(s)) d s \\
& \leq \mathcal{E}_{N}\left(U_{0}\right)+l \int_{0}^{t}(1+s)^{l-1} \mathcal{E}_{N} U(s) d s \\
& \leq \mathcal{E}_{N}\left(U_{0}\right)+C l \int_{0}^{t}(1+s)^{l-1}\left(\mathcal{D}_{N-1} U(s)+\mathcal{D}_{N}^{h}(U(s))+\|u(s)\|_{L^{2}}^{2}\right) d s,
\end{aligned}
$$

where we have used

$$
\mathcal{E}_{N} U(t) \leq C \mathcal{D}_{N-1} U(t)+C \mathcal{D}_{N}^{h}(U(t))+\|u(t)\|_{L^{2}}^{2} .
$$


Using (5.5) again, we have

$$
\mathcal{E}_{N+1}(U(t))+\lambda_{1} \int_{0}^{t} \mathcal{D}_{N+1}(U(t))+\lambda_{2} \int_{0}^{t} \mathcal{D}_{N+1}^{h}(U(t)) \leq \mathcal{E}_{N+1}\left(U_{0}\right),
$$

and

$$
\begin{aligned}
(1+t)^{l-1} \mathcal{E}_{N+1} U(t)+\lambda_{1} \int_{0}^{t}(1+s)^{l-1} \mathcal{D}_{N+1}(U(s)) d s+\lambda_{2} \int_{0}^{t}(1+s)^{l-1} \mathcal{D}_{N+1}^{h}(U(s)) d s \\
\quad \leq \mathcal{E}_{N+1}\left(U_{0}\right)+C(l-1) \int_{0}^{t}(1+s)^{l-2} \mathcal{E}_{N+1} U(s) d s \\
\quad \leq \mathcal{E}_{N+1}\left(U_{0}\right)+C(l-1) \int_{0}^{t}(1+s)^{l-2}\left(\mathcal{D}_{N} U(s)+C \mathcal{D}_{N+1}^{h}(U(s))+\|u(s)\|_{L^{2}}^{2}\right) d s
\end{aligned}
$$

By iterating the above estimates for $1<l<2$, we have

$$
\begin{aligned}
(1+t)^{l} \mathcal{E}_{N} U(t)+ & \lambda_{1} \int_{0}^{t}(1+s)^{l} \mathcal{D}_{N}(U(s)) d s+\lambda_{2} \int_{0}^{t}(1+s)^{l} \mathcal{D}_{N}^{h}(U(s)) d s \\
\leq & \mathcal{E}_{N+1}\left(U_{0}\right)+C \int_{0}^{t}(1+s)^{l-1}\|u(s)\|_{L^{2}}^{2} d s
\end{aligned}
$$

To estimate the integral term on the right-hand side of (5.6), let us define

$$
\mathcal{E}_{N, \infty}(U(t))=\sup _{0 \leq s \leq T}(1+t)^{\frac{3}{2}} \mathcal{E}_{N} U(t)
$$

Now, we estimate the integral term on the right-hand side of (5.6) by applying the linear estimate on $u$ in (4.38) to the mild form (5.1), giving us

$$
\begin{aligned}
\|u(t)\|_{L^{2}} & \leq C(1+t)^{\frac{-3}{4}}\left\|\rho_{0}, u_{0}\right\|_{L^{1}}+C e^{-\lambda t}\left\|\rho_{0}, u_{0}\right\|_{L^{2}} \\
& \left.+C \int_{0}^{t}(1+t-s)^{\frac{-3}{4}}\left\|g_{1}, g_{2}\right\|_{L^{1}} d s+C \int_{0}^{t} e^{-\lambda(t-s)}\right)\left\|g_{1}, g_{2}\right\|_{L^{2}} d s .
\end{aligned}
$$

Recall the definitions (4.3) of $g_{1}$ and $g_{2}$. It is direct to check that for any $0 \leq s \leq t$,

$$
\left\|g_{1}(s), g_{2}(s)\right\|_{L^{1} \cap L^{2}} \leq C \mathcal{E}_{N} U(t) \leq C(1+s)^{\frac{-3}{2}} \mathcal{E}_{N, \infty} U(t)
$$

where

$$
\mathcal{E}_{N, \infty}(U(t))=\sup _{0 \leq s \leq T}(1+t)^{\frac{3}{2}} \mathcal{E}_{N} U(t)
$$

Putting the above inequalities into (5.7), gives

$$
\|u(t)\|_{L^{2}} \leq C(1+t)^{\frac{-3}{4}}\left(\left\|\rho_{0}, u_{0}\right\|_{L^{1} \cap L^{2}}+\mathcal{E}_{N, \infty} U(t)\right) .
$$


Next, we prove the uniform-in-time boundedness of $\mathcal{E}_{N, \infty} U(t)$ which yields the time-decay rates of the energy functional $\mathcal{E}_{N} U(t)$. In fact, by taking $l=\frac{3}{2}+\epsilon$ in (5.6) where $\epsilon>0$ is sufficiently small, it follows that

$$
\begin{gathered}
(1+t)^{\frac{3}{2}+\epsilon} \mathcal{E}_{N} U(t)+\lambda_{1} \int_{0}^{t}(1+s)^{\frac{3}{2}+\epsilon} \mathcal{D}_{N}(U(s)) d s+\lambda_{2} \int_{0}^{t}(1+s)^{\frac{3}{2}+\epsilon} \mathcal{D}_{N}^{h}(U(s)) d s \\
\leq \mathcal{E}_{N+1}\left(U_{0}\right)+C \int_{0}^{t}(1+s)^{\frac{1}{2}+\epsilon}\|u(s)\|_{L^{2}}^{2} d s .
\end{gathered}
$$

Here, using (5.10) and the fact that $\mathcal{E}_{N, \infty}(U(t))$ is non-decreasing in t, it further holds that

$$
\left.\int_{0}^{t}(1+s)^{\frac{1}{2}+\epsilon}\|u(t)\|_{L^{2}}^{2} d s \leq C(1+t)^{\epsilon}\left(\mathcal{E}_{N, \infty}^{2} U(t)\right)+\left\|\rho_{0}, u_{0}\right\|_{L^{1} \cap L^{2}}^{2}\right) .
$$

Therefore, it follows that

$$
\begin{aligned}
(1+t)^{\frac{3}{2}+\epsilon} \mathcal{E}_{N} U(t)+ & \lambda_{1} \int_{0}^{t}(1+s)^{\frac{3}{2}+\epsilon} \mathcal{D}_{N}(U(s)) d s+\lambda_{2} \int_{0}^{t}(1+s)^{\frac{3}{2}+\epsilon} \mathcal{D}_{N}^{h}(U(s)) d s \\
& \left.\leq \mathcal{E}_{N+1}\left(U_{0}\right)+C(1+t)^{\epsilon}\left(\mathcal{E}_{N, \infty}^{2} U(t)\right)+\left\|\rho_{0}, u_{0}\right\|_{L^{1} \cap L^{2}}^{2}\right)
\end{aligned}
$$

which implies

$$
(1+t)^{\frac{3}{2}} \mathcal{E}_{N} U(t) \leq C\left(\mathcal{E}_{N+1}\left(U_{0}\right)+\left\|\rho_{0}, u_{0}\right\|_{L^{1}}^{2}+\mathcal{E}_{N, \infty}^{2} U(t)\right)
$$

and thus

$$
\mathcal{E}_{N, \infty} U(t) \leq C\left(\epsilon_{N+1}^{2}\left(U_{0}\right)+\mathcal{E}_{N, \infty}^{2} U(t)\right)
$$

Since $\epsilon_{N+1}\left(U_{0}\right)>0$ is sufficiently small, it holds that $\left.\mathcal{E}_{N, \infty} U(t)\right) \leq C \epsilon_{N+1}^{2}\left(U_{0}\right)$ for any $t \geq 0$, which gives $\|U(s)\|_{H^{N}} \leq C\left(\mathcal{E}_{N} U(t)\right)^{\frac{1}{2}} \leq C \epsilon_{N+1}\left(U_{0}\right)(1+t)^{-\frac{3}{4}}$. This proves (5.3).

Now, we estimate the high-order energy functional. By comparing the definitions of $\mathcal{E}_{N} U(t)$, $\mathcal{D}_{N} U(t)$ and $\mathcal{D}_{N}^{h} U(t)$, it follows from (5.5) that we have

$$
\frac{d}{d t}\|\nabla U(t)\|_{H^{N}}^{2}+\lambda\|\nabla U(t)\|_{H^{N}}^{2} \leq C\|\nabla u(t)\|_{L^{2}}^{2}
$$

which implies

$$
\|\nabla U(t)\|_{H^{N}}^{2} \leq e^{-\lambda t}\left\|\nabla U_{0}\right\|_{H^{N}}^{2}+C \int_{0}^{t} e^{-\lambda(t-s)}\|\nabla u(s)\|_{L^{2}}^{2} d s,
$$


for any $t \geq 0$.

Similarly to obtaining (5.8), we estimate the time integral term on the (r.h.s.) of the above inequality. One can apply the linear estimate (4.29) to the mild form (5.1) so that

$$
\begin{aligned}
& \|\nabla u(t)\|_{L^{2}} \leq C(1+t)^{\frac{-5}{4}}\left\|\rho_{0}, u_{0}\right\|_{L^{1}}+C e^{-\lambda t}\left\|\left[\rho_{0}, u_{0}\right]\right\|_{\dot{H}^{1}} \\
& \quad+C \int_{0}^{t}(1+t-s)^{\frac{-5}{4}}\left\|\left[g_{1}(s), g_{2}(s)\right]\right\|_{L^{1}} d s+C \int_{0}^{t} e^{-\lambda(t-s)}\left\|\left[g_{1}(s), g_{2}(s)\right]\right\|_{\dot{H}^{1}} d s .
\end{aligned}
$$

Recall the definition (4.3) of $g_{1}$ and $g_{2}$. It is straightforward to check that for any $0 \leq s \leq t$,

$$
\left\|\left[g_{1}(s), g_{2}(s)\right]\right\|_{L^{1} \cap \dot{H}^{1}} \leq C \mathcal{E}_{N} U(s) \leq C \epsilon_{N+1}^{2}\left(U_{0}\right)(1+s)^{\frac{-3}{2}} .
$$

Putting this into (5.10) gives

$$
\|\nabla u(t)\|_{L^{2}} \leq C \epsilon_{N+1}\left(U_{0}\right)(1+t)^{\frac{-5}{4}}
$$

Then, by using (5.11) in (5.9), we have

$$
\|\nabla U(t)\|_{H^{N}}^{2} \leq e^{-\lambda t}\left\|\nabla U_{0}\right\|_{H^{N}}^{2}+C \epsilon_{N+1}^{2}\left(U_{0}\right)(1+t)^{\frac{-5}{2}}
$$

which implies (5.4). The proof of Proposition 5.0.1 is complete.

\subsubsection{Time-decay rate in $L^{q}$}

In this subsection, we will prove Proposition 2.0.2 for time-decay rates in $L^{q}$ with $2 \leq q \leq \infty$

corresponding to (1.4)-(1.6) in Theorem 1.1. For $N \geq 4$, Proposition 5.0.1 shows that if $\epsilon_{N+1}\left(U_{0}\right)$ is small enough,

$$
\|U(s)\|_{H^{N}} \leq C \epsilon_{N+1}\left(U_{0}\right)(1+t)^{-\frac{3}{4}}
$$

and

$$
\|\nabla U(t)\|_{H^{N}} \leq C \epsilon_{N+1}\left(U_{0}\right)(1+t)^{\frac{-5}{4}}
$$

Now, let us establish the estimates on $u, \rho$ as follows.

Estimate on $\|u(t)\|_{L^{q}}$. For the $L^{2}$ rate, it is easy to see from (5.8) and (5.12) that

$$
\|u(t)\|_{L^{2}} \leq C \epsilon_{N+1}\left(U_{0}\right)(1+t)^{\frac{-3}{4}} \leq C(1+t)^{\frac{-3}{4}}
$$


For the $L^{\infty}$ rate, by applying the $L^{\infty}$ linear estimate on $u$ in (4.40) to the mild form (5.1), we have

$$
\begin{aligned}
\|u(t)\|_{L^{\infty}} & \leq C(1+t)^{\frac{-3}{2}}\left\|\rho_{0}, u_{0}\right\|_{L^{1}}+C e^{-\lambda t}\left\|\nabla^{2}\left[\rho_{0}, u_{0}\right]\right\|_{L^{2}} \\
& +C \int_{0}^{t}(1+t-s)^{\frac{-3}{2}} \|\left[\left[g_{1}(s), g_{2}(s)\right]\left\|_{L^{1}} d s+C \int_{0}^{t} e^{-\lambda(t-s)}\right\| \nabla^{2}\left[g_{1}(s), g_{2}(s)\right] \|_{L^{2}} d s\right. \\
& \leq C(1+t)^{\frac{-3}{2}}\left\|\rho_{0}, u_{0}\right\|_{L^{1} \cap \dot{H}^{2}}+C \int_{0}^{t}(1+t-s)^{\frac{-3}{2}}\left\|\left[g_{1}(s), g_{2}(s)\right]\right\|_{L^{1} \cap \dot{H}^{2}} d s .
\end{aligned}
$$

Since by (5.12) and (5.13)

$$
\left\|\left[g_{1}(s), g_{2}(s)\right]\right\|_{L^{1} \cap \dot{H}^{2}} \leq C\|\nabla U(t)\|_{H^{N}}\|U(s)\|_{H^{N}} \leq C \epsilon_{N+1}^{2}\left(U_{0}\right)(1+s)^{-2},
$$

it follows that

$$
\|u(t)\|_{L^{\infty}} \leq C \epsilon_{N+1}\left(U_{0}\right)(1+t)^{\frac{-3}{2}} .
$$

Then, by $L^{2}-L^{\infty}$ interpolation,

$$
\|u\|_{L^{q}} \leq C \epsilon_{N+1}\left(U_{0}\right)(1+t)^{\frac{-3}{2}+\frac{3}{2 q}}
$$

for $2 \leq q \leq \infty$.

Estimate on $\|\rho(t)\|_{L^{q}}$. For the $L^{2}$ rate, utilizing the $L^{2}$ estimate on $\rho$ in (4.37) to (5.1), we have

$$
\begin{aligned}
\|\rho(t)\|_{L^{2}} \leq & C(1+t)^{\frac{-5}{4}}\left\|\rho_{0}, u_{0}\right\|_{L^{1}}+C e^{-\lambda t}\left\|\rho_{0}, u_{0}\right\|_{L^{2}}+C \int_{0}^{t}(1+t-s)^{\frac{-5}{4}}\left\|g_{1}, g_{2}\right\|_{L^{1}} d s \\
& +C \int_{0}^{t} e^{-\lambda(t-s)}\left\|g_{1}(s), g_{2}(s)\right\|_{L^{2}} d s
\end{aligned}
$$

Due to (5.12),

$$
\left\|g_{1}(s), g_{2}^{*}(s)\right\|_{L^{1} \cap L^{2}} \leq C\|U(s)\|_{H^{N}}^{2} \leq C \epsilon_{N+1}^{2}\left(U_{0}\right)(1+t)^{\frac{-3}{2}} .
$$

Then (5.16) implies the slower decay estimate

$$
\|\rho(t)\|_{L^{2}} \leq C \epsilon_{N+1}\left(U_{0}\right)(1+t)^{\frac{-5}{4}} \leq C(1+t)^{\frac{-5}{4}} .
$$

For the $L^{\infty}$ rate, utilizing the $L^{\infty}$ estimate on $\rho$ in (4.39) to (5.1), we have

$$
\|\rho(t)\|_{L^{\infty}} \leq(1+t)^{-2}\left\|\rho_{0}, u_{0}\right\|_{L^{1} \cap \dot{H}^{2}}+C \int_{0}^{t}(1+t-s)^{-2}\left\|\left[g_{1}(s), g_{2}(s)\right]\right\|_{L^{1} \cap \dot{H}^{2}} d s .
$$


Since by (5.12) and (5.13)

$$
\left\|\left[g_{1}(s), g_{2}(s)\right]\right\|_{L^{1} \cap \dot{H}^{2}} \leq C\|\nabla U(t)\|_{H^{N}}\|U(s)\|_{H^{N}} \leq C \epsilon_{N+1}^{2}\left(U_{0}\right)(1+s)^{-2},
$$

which yields from (5.18) that

$$
\|\rho(t)\|_{L^{\infty}} \leq C \epsilon_{N+1}\left(U_{0}\right)(1+s)^{-2} .
$$

Therefore, by $L^{2}-L^{\infty}$ interpolation,

$$
\|\rho(t)\|_{L^{q}} \leq C \epsilon_{N+1}\left(U_{0}\right)(1+s)^{-2+\frac{3}{2 q}}
$$

for $2 \leq q \leq \infty$.

Next, we estimate the time-decay rate of $\left[c_{1}, c_{2}\right]$. We start with the estimate on $\left\|c_{1}(t)\right\|_{L^{q}}$. For the $L^{2}$ rate,

$$
\begin{aligned}
& \left\|c_{1}\right\|_{L^{2}} \leq C\left\|\hat{c}_{1}\right\|_{L^{2}(\xi)} \leq C\left[\int_{\xi} e^{-2\left(|\xi|^{2}+\left(a_{12}-a_{11} n_{\infty}\right)\right) t}\left|\hat{c}_{0}\right|^{2} d \xi\right]^{\frac{1}{2}} \\
& +a_{11} \int_{0}^{t}\left[\int_{\xi}\left[e^{-2\left(|\xi|^{2}+\left(a_{12}-a_{11} n_{\infty}\right)\right)(t-s)}\left|\rho \hat{c}_{1}\right|^{2} d \xi\right]^{\frac{1}{2}} d s\right. \\
& \leq e^{-\left(a_{12}-a_{11} n_{\infty}\right) t}\left[\int_{\xi} e^{-2|\xi|^{2}(t)}\left|\hat{c}_{0}\right|^{2} d \xi\right]^{\frac{1}{2}}+C \int_{0}^{t} e^{-\left(a_{12}-a_{11} n_{\infty}\right)(t-s)}\left[\int_{\xi} e^{-2|\xi|^{2}(t-s+1)}\left|\rho \hat{c}_{1}\right|^{2} d \xi\right]^{\frac{1}{2}} d s \\
& \leq C e^{-\left(a_{12}-a_{11} n_{\infty}\right) t}\left\|\hat{c}_{0}\right\|_{L^{2}}+C \int_{0}^{t} e^{-\left(a_{12}-a_{11} n_{\infty}\right)(t-s)} \sup _{\xi} e^{-|\xi|^{2}(t-s+1)}\left\|\rho c_{1}(s)\right\|_{L^{2}} d s
\end{aligned}
$$

Due to (5.12),

$$
\left\|\rho c_{1}(s)\right\|_{L^{2}} \leq C\|U(s)\|_{N}^{2} \leq C \epsilon_{N+1}^{2}\left(U_{0}\right)(1+t)^{\frac{-3}{2}} .
$$

Then (5.20) implies the slower decay estimate

$$
\left\|c_{1}\right\|_{L^{2}} \leq C \epsilon_{N+1}\left(U_{0}\right)(1+t)^{\frac{-3}{2}} .
$$

Similarly, we have

$$
\left\|c_{2}\right\|_{L^{2}} \leq C \epsilon_{N+1}\left(U_{0}\right)(1+t)^{\frac{-3}{2}} .
$$


For $L^{\infty}$ rate, from the Hausdorff-Young inequality and Hölder inequality, we have

$$
\begin{aligned}
& \left\|c_{1}\right\|_{L^{\infty}} \leq C\left\|\hat{c}_{1}\right\|_{L^{1}} \leq C \int_{\xi \leq \epsilon} e^{-\left(|\xi|^{2}+\left(a_{12}-a_{11} n_{\infty}\right)\right) t}\left|\hat{c}_{1,0}\right| d \xi \\
& +C \int_{0}^{t} \int_{\xi \leq \epsilon} e^{-\left(|\xi|^{2}+\left(a_{12}-a_{11} n_{\infty}\right)\right)(t-s)}\left|\rho \hat{c}_{1}\right| d \xi d s \\
& +C \int_{|\xi| \geq \epsilon} e^{\left.-\left(a_{12}-a_{11} n_{\infty}\right)\right) t}\left|\hat{c}_{1,0}\right| d \xi+C \int_{0}^{t} \int_{|\xi| \geq \epsilon} e^{\left.-\left(a_{12}-a_{11} n_{\infty}\right)\right)(t-s)}\left|\rho \hat{c}_{1}\right| d \xi d s \\
& \leq C e^{-\left(a_{12}-a_{11} n_{\infty}\right) t}(1+t)^{\frac{-3}{2}}\left\|c_{0}\right\|_{L^{1}}+C \int_{0}^{t} e^{-\left(a_{12}-a_{11} n_{\infty}\right)(t-s)}\left\|\rho \hat{c}_{1}(s)\right\|_{L^{1}} \\
& +C e^{\left.-\left(a_{12}-a_{11} n_{\infty}\right)\right) t}\left[\int_{|\xi| \geq \epsilon}|\xi|^{-4} d \xi\right]^{\frac{1}{2}}\left[\int_{|\xi| \geq \epsilon}|\xi|^{4}\left|\hat{c}_{1,0}\right|^{2} d \xi\right]^{\frac{1}{2}} \\
& +C \int_{0}^{t} e^{\left.-\left(a_{12}-a_{11} n_{\infty}\right)\right)(t-s)}\left[\int_{|\xi| \geq \epsilon}|\xi|^{-4} d \xi\right]^{\frac{1}{2}}\left[\int_{|\xi| \geq \epsilon}|\xi|^{4}\left|\rho \hat{c}_{1}\right|^{2} d \xi\right]^{\frac{1}{2}} d s \\
& \leq C e^{-\left(a_{12}-a_{11} n_{\infty}\right) t}(1+t)^{\frac{-3}{2}}\left\|c_{0}\right\|_{L^{1}}+C \int_{0}^{t} e^{-\left(a_{12}-a_{11} n_{\infty}\right)(t-s)}\left\|\rho c_{1}(s)\right\|_{L^{1}} d s \\
& +C e^{\left.-\left(a_{12}-a_{11} n_{\infty}\right)\right) t}\left\|\nabla^{2} c_{0}\right\|_{L^{2}}+C \int_{0}^{t} e^{-\left(a_{12}-a_{11} n_{\infty}\right)(t-s)}\left\|\nabla^{2}\left(\rho c_{1}(s)\right)\right\|_{L^{2}} d s
\end{aligned}
$$

Since by $(5.12)$

$$
\left\|\rho c_{1}(s)\right\|_{L^{1} \cap \dot{H}^{2}} \leq C\|U(s)\|_{N}^{2} \leq C \epsilon_{N+1}^{2}\left(U_{0}\right)(1+t)^{\frac{-3}{2}} .
$$

Then, (5.23) implies the slower decay estimate

$$
\left\|c_{1}\right\|_{L^{\infty}} \leq C \epsilon_{N+1}\left(U_{0}\right)(1+t)^{\frac{-3}{2}} .
$$

Similarly, we have

$$
\left\|c_{2}\right\|_{L^{\infty}} \leq C \epsilon_{N+1}\left(U_{0}\right)(1+t)^{\frac{-3}{2}} .
$$

So, by $L^{2}-L^{\infty}$ interpolation,

$$
\left\|\left[c_{1}, c_{2}\right]\right\|_{L^{q}} \leq C \epsilon_{N+1}\left(U_{0}\right)(1+t)^{\frac{-3}{2}},
$$

for $2 \leq q \leq \infty$.

This completes the proof of Proposition 2.0.2 and hence Theorem 1.0.1. 


\section{Part II}

\section{Global Existence and Decay Rates of}

the Solutions for a Chemotaxis

System with Lotka-Volterra Type

Model for Chemo Agents 


\section{Chapter 6}

\section{Introduction}

We consider the initial value problem of the system in $\mathbb{R}^{3}$

$$
\left\{\begin{array}{l}
\partial_{t} n+\nabla \cdot(n u)=0 \\
\partial_{t}(n u)+\nabla \cdot(n u \otimes u+p(n))=n\left(\nabla c_{1}-\nabla c_{2}\right)-\nu n u \\
\partial_{t} c_{1}=\Delta c_{1}+c_{1}\left(-a_{1}+a_{11} c_{1}+a_{12} c_{2}+a_{13} n\right) \\
\partial_{t} c_{2}=\Delta c_{2}+c_{2}\left(-a_{2}+a_{21} c_{1}+a_{22} c_{2}+a_{23} n\right)
\end{array}\right.
$$

where $a_{1}>0, a_{2}>0$. The signs of the other coefficients are generally given as

$$
\begin{aligned}
& a_{11}>0, a_{12}<0, a_{13} \geq 0, \\
& a_{21}<0, a_{22}>0, a_{23} \geq 0 .
\end{aligned}
$$

The initial data is given by

$$
\left.\left(n, u, c_{1}, c_{2}\right)\right|_{t=0}=\left(n_{0}, u_{0}, c_{1,0}, c_{2,0}\right)(x), \quad x \in \mathbb{R}^{3} .
$$

The first two equations are the conservation of mass and momentum for the cells. In angiogenesis or vasculogenesis blood vessels are the cells. The gradients of $c_{1}$ and $c_{2}$ cause cells to grow toward and away from the higher density of $c_{1}$ and $c_{2}$, respectively. For this reason, they are called attractants and repellents, respectively. In this paper, we use the reaction-diffusion equations for them, and for the interactions among them and cells we use a Lotka-Volterra type competitive model. An example of an attractant in angiogenesis or vasculogenesis is the vascular endothelial growth factor (VEGF) and it is a signal protein 
produced by cancer cells that stimulate the formation of blood vessels. An example of repellent can be anti-VEGF medications that block VEGF.

Subtracting the first equation from the second equation, we will consider the simplified chemotaxis fluid equations taking the following form

$$
\left\{\begin{array}{l}
\partial_{t} n+\nabla \cdot(n u)=0 \\
\partial_{t} u+u \cdot \nabla u+\frac{\nabla p(n)}{n}=\nabla c_{1}-\nabla c_{2}-\nu u \\
\partial_{t} c_{1}=\Delta c_{1}-a_{1} c_{1}+a_{11} c_{1}^{2}+a_{12} c_{1} c_{2}+a_{13} c_{1} n \\
\partial_{t} c_{2}=\Delta c_{2}-a_{2} c_{2}+a_{21} c_{1} c_{2}+a_{22} c_{2}^{2}+a_{23} c_{2} n
\end{array}\right.
$$

with initial data

$$
\left.\left(n, u, c_{1}, c_{2}\right)\right|_{t=0}=\left(n_{0}, u_{0}, c_{1,0}, c_{2,0}\right)(x), \quad x \in \mathbb{R}^{3} .
$$

$\left(n_{0}, u_{0}, c_{1,0}, c_{2,0}\right)(x) \rightarrow\left(n_{\infty}, 0,0,0\right) \quad$ as $\quad|x| \rightarrow \infty$, for some constant $n_{\infty}>0$. Throughout this paper, we assume the following: $p($.$) is the smooth function of n$ and $p^{\prime}(n)>0$. The main goals of this paper are to show the local and global existence of solutions in $H^{N}\left(\mathbb{R}^{3}\right)$ and $L^{q}$ time-decay rates of solutions for the Cauchy problem for the above system (6.1)-(6.2). The main result of this paper is stated as follows.

Theorem 6.0.1. Let $N \geq 4$. There exists a positive number $\epsilon_{0}$ such that if

$$
\left\|\left[n_{0}-n_{\infty}, u_{0}, c_{1,0}, c_{2,0}\right]\right\|_{H^{N}} \leq \epsilon_{0}
$$

the Cauchy problem (6.3)-(6.4) has a unique solution $\left(n, u, c_{1}, c_{2}\right)(t)$ globally in time which satisfies

$$
\begin{array}{r}
\left(n-n_{\infty}, u\right)(t) \in C\left([0, \infty) ; H^{N}\left(\mathbb{R}^{3}\right)\right) \cap C^{1}\left([0, \infty) ; H^{N-1}\left(\mathbb{R}^{3}\right)\right), \\
\left(c_{1}, c_{2}\right)(t) \in C\left([0, \infty) ; H^{N}\left(\mathbb{R}^{3}\right)\right) \cap C^{1}\left([0, \infty) ; H^{N-2}\left(\mathbb{R}^{3}\right)\right)
\end{array}
$$

and there are constants $\lambda_{1}>0, \lambda_{2}>0, \lambda_{3}>0$ and $C_{0}>0$ such that

$$
\begin{aligned}
\left\|\left[n-n_{\infty}, u, c_{1}, c_{2}\right]\right\|_{H^{N}}^{2} & +\lambda_{1} \int_{0}^{t}\left\|\nabla\left[n-n_{\infty}\right]\right\|_{H^{N-1}}^{2}+\lambda_{2} \int_{0}^{t}\left\|\nabla\left[c_{1}, c_{2}\right]\right\|_{H^{N}}^{2} \\
& +\lambda_{3} \int_{0}^{t}\left\|\left[u, c_{1}, c_{2}\right]\right\|_{H^{N}}^{2} \leq C_{0}\left\|\left[n_{0}-n_{\infty}, u_{0}, c_{1,0}, c_{2,0}\right]\right\|_{H^{N}}^{2} .
\end{aligned}
$$


Moreover, the global solution $\left[n, u, c_{1}, c_{2}\right]$ obtained above satisfies for $t \geq t_{0}$ with $t_{0}>0 a$ sufficiently large time that:

$$
\begin{gathered}
\left\|n-n_{\infty}\right\|_{L^{q}} \leq C(1+t)^{\frac{-3}{2}+\frac{3}{2 q}}, \\
\|u\|_{L^{q}} \leq C(1+t)^{\frac{-3}{2}+\frac{1}{2 q}} \\
\left\|\left(c_{1}, c_{2}\right)\right\|_{L^{q}} \leq C(1+t)^{\frac{-3}{2}}
\end{gathered}
$$

with $2 \leq q \leq \infty$, where $C>0$ is a positive constant independent of time.

The proof of the existence of global solutions in Theorem 6.0.1 is based on the local existence and an a priori estimate. The local existence can be proved by constructing a sequence of approximation functions based on an iteration by following the methods in Kato [16] and Majda [17] . The a priori estimate can be obtained by the energy method. Moreover, to obtain the time-decay rate in $L^{q}$ norm of solutions in Theorem 6.0.1, our approach is a combined analysis of Green's function of the linear system and the refined energy estimates with the help of Duhamel's principle. We obtain Green's matrix of the linear system by Fourier transform.

We mention some previous related works about chemotaxis models. Such chemotaxis models are based on the Keller-Segel system. Wang [5] explored the interactions between the nonlinear diffusion and logistic source on the solutions of the attraction-repulsion chemotaxis system in three dimensions. E. Lankeit and J. Lankeit [6] proved the global existence of classical solutions to a chemotaxis system with singular sensitivity. Liu and Wang [7] established the existence of global classical solutions and steady states to an attractionrepulsion chemotaxis model in one dimension based on the method of energy estimates. Luca, Chavez-Ross, Edelstein-Keshet, and Mogilner [18] investigated conditions that lead to aggregation of microglia and developed a model for chemotaxis in response to a combination of chemoattractant and chemorepellent signaling chemicals.

Concerning the chemotaxis models based on fluid dynamics, there are two approaches, incompressible and compressible. For the incompressible case, Chae, Kang and Lee [8], and Duan, Lorz, and Markowich [9] showed the global-in-time existence for the incompressible chemotaxis equations near the constant states, if the initial data is sufficiently small. 
Rodriguez, Ferreira and Villamizar-Roa [10] showed the global existence for an attractionrepulsion chemotaxis fluid model with a logistic source. Tan and Zhou [11] proved the global existence and time-decay estimate of solutions to the Keller-Segel system in $R^{3}$ with small initial data.

For the compressible case, Ambrosi, Bussolino, and Preziosi [12] discussed the vasculogenesis using the compressible fluid dynamics for the cells and the diffusion equation for the attractant.

Many related approaches that use Fourier transform, and we only mention that Duan [13] and Duan, Liu, and Zhu [14] proved the time-decay rate by the combination of energy estimates and spectral analysis.

For later use in this paper, we give some notations. C denotes some positive constant, $\lambda_{i}$, where $i=1,2$, denotes some positive (generally small) constant, where both $\mathrm{C}$ and $\lambda_{i}$ may take different values in different places. For any integer $m \geq 0$, we use $H^{m}$ to denote the Sobolev space $H^{m}\left(\mathbb{R}^{3}\right)$ and $\dot{H}^{m}$ the $m^{\text {th }}$-order homogeneous Sobolev space. Set $L^{2}=H^{0}$. For simplicity, the norm of $H^{m}$ is denoted by $\|\cdot\|_{m}$ with $\|\cdot\|=\|\cdot\|_{0}$. We set $\partial^{\alpha}=\partial_{x_{1}}^{\alpha_{1}} \partial_{x_{2}}^{\alpha_{2}} \partial_{x_{3}}^{\alpha_{3}}$ for a multi-index $\alpha=\left[\alpha_{1}, \alpha_{2}, \alpha_{3}\right]$. The length of $\alpha$ is $||=.\alpha_{1}+\alpha_{2}+\alpha_{3}$ and we also set $\partial_{j}=\partial_{x_{j}}$ for $j=1,2,3$. For an integrable function $f: \mathbb{R}^{3} \rightarrow \mathbb{R}$, its Fourier transform is defined by $\hat{f}=\int_{\mathbb{R}^{3}} e^{-i x \cdot \xi} f(x) d x, \quad x \cdot \xi=\sum_{i=0}^{3} x_{j} \xi_{j}, \quad x \in \mathbb{R}^{3}$, where $i=\sqrt{-1}$ is the imaginary unit. Let us denote the space

$$
\begin{aligned}
X(0, T)= & \left\{(\rho, u) \in C\left([0, T] ; H^{N}\left(\mathbb{R}^{3}\right)\right) \cap C^{1}\left([0, T] ; H^{N-1}\left(\mathbb{R}^{3}\right)\right),\right. \\
& \left.\left(c_{1}, c_{2}\right) \in C\left([0, T] ; H^{N}\left(\mathbb{R}^{3}\right)\right) \cap C^{1}\left([0, T] ; H^{N-2}\left(\mathbb{R}^{3}\right)\right)\right\} .
\end{aligned}
$$

This paper is organized as follows. In chapter 7, we reformulate the Cauchy problem under consideration. In chapter 8, we prove the global existence and uniqueness of solutions. In chapter 9 , we investigate the linearized homogeneous system to obtain the $L^{q}$ time-decay property and the explicit representation of solutions. In chapter 10 , we study the $L^{q}$ timedecay rates of solutions to the reformulated nonlinear system and finish the proof of Theorem 6.0.1. 


\section{Chapter 7}

\section{Reformulation of the system (6.3)}

Let $U(t)=\left[n, u, c_{1}, c_{2}\right]$ be a smooth solution to the Cauchy problem of the chemotaxis fluid equations (6.3) with initial data $U_{0}=\left[n_{0}, u_{0}, c_{1,0}, c_{2,0}\right]$. Set

$$
n(x, t)=\rho(x, t)+n_{\infty} .
$$

Then the Cauchy problem (6.3)-(6.4) are reformulated as

$$
\left\{\begin{array}{l}
\partial_{t} \rho+n_{\infty} \nabla \cdot u=-\nabla \cdot(\rho u) \\
\partial_{t} u+u \cdot \nabla u+\nu u+\frac{p^{\prime}\left(\rho+n_{\infty}\right)}{\rho+n_{\infty}} \nabla \rho=\nabla c_{1}-\nabla c_{2} \\
\partial_{t} c_{1}-\Delta c_{1}+\left(a_{1}-a_{13} n_{\infty}\right) c_{1}=a_{11} c_{1}^{2}+a_{12} c_{1} c_{2}+a_{13} c_{1} \rho \\
\partial_{t} c_{2}-\Delta c_{2}+\left(a_{2}-a_{23} n_{\infty}\right) c_{2}=a_{21} c_{1} c_{2}+a_{22} c_{2}^{2}+a_{23} c_{2} \rho
\end{array}\right.
$$

with initial data

$$
\left.\left(\rho, u, c_{1}, c_{2}\right)\right|_{t=0}=\left(\rho_{0}, u_{0}, c_{1,0}, c_{2,0}\right) \rightarrow(0,0,0,0),
$$

as $|x| \rightarrow \infty$, where $\rho_{0}=n_{0}-n_{\infty}$. We assume that $\left(a_{12}-n_{\infty} a_{11}\right)>0$ and $\left(a_{22}-n_{\infty} a_{21}\right)>0$. In the following, we set $N \geq 4$. Besides, for $U=\left[\rho, u, c_{1}, c_{2}\right]$, we use $\mathcal{E}_{N} U(t)$ to denote the energy functional and $\mathcal{D}_{N} U(t), \mathcal{D}_{N}^{h} U(t)$ the dissipation rates. Here,

$$
\begin{aligned}
& \mathcal{E}_{N} U(t) \sim\left\|\left[\rho, u, c_{1}, c_{2}\right]\right\|_{N}^{2}, \\
& \mathcal{D}_{N} U(t) \sim\left\|\left[\nabla\left(c_{1}, c_{2}\right)\right]\right\|_{N}^{2},
\end{aligned}
$$

and

$$
\mathcal{D}_{N}^{h} U(t) \sim\|[\nabla \rho]\|_{N-1}^{2}+\left\|\left[u, c_{1}, c_{2}\right]\right\|_{N}^{2} .
$$


Then, concerning the reformulated Cauchy problem (7.2)-(7.3), one has the following global existence result.

Proposition 7.0.1. Suppose that $\left\|\left[\rho_{0}, u_{0}, c_{1,0}, c_{2,0}\right]\right\|_{H^{N}}$ is sufficiently small. Then, the Cauchy problem (7.2)-(7.3) has a unique solution $U(t)=\left(\rho, u, c_{1}, c_{2}\right)(t)$ globally in time which satisfies $U(t) \in X(0, \infty)$ and

$$
\mathcal{E}_{N}(U(t))+\lambda_{1} \int_{0}^{t} \mathcal{D}_{N}(U(t)) d s+\lambda_{2} \int_{0}^{t} \mathcal{D}_{N}^{h}(U(t)) d s \leq C_{0} \mathcal{E}_{N}\left(U_{0}\right),
$$

for any $t \geq 0$.

Moreover, the solutions obtained in Proposition 7.0.1 indeed have the decay rates in time under some extra conditions on the initial data. For that, given $U_{0}=\left[\rho_{0}, u_{0}, c_{1,0}, c_{2,0}\right]$, set $\epsilon_{N}\left(U_{0}\right)$ as

$$
\epsilon_{N}\left(U_{0}\right)=\left\|U_{0}\right\|_{N}+\left\|U_{0}\right\|_{L^{1}}
$$

for $N \geq 4$. Then, we have the following two Propositions:

Proposition 7.0.2. Let $U=\left[\rho, u, c_{1}, c_{2}\right]$ be the solution to the Cauchy problem (7.2) with initial data $U_{0}=\left(\rho_{0}, u_{0}, c_{1,0}, c_{2,0}\right)$. If $\epsilon_{N+1}\left(U_{0}\right)>0$ is sufficiently small, then the solution $U=\left[\rho, u, c_{1}, c_{2}\right]$ satisfies

$$
\|U(t)\|_{N} \leq \epsilon_{N+1}\left(U_{0}\right)(1+t)^{\frac{-3}{4}}
$$

and

$$
\|\nabla U(t)\|_{N} \leq \epsilon_{N+1}\left(U_{0}\right)(1+t)^{\frac{-5}{4}}
$$

for any $t \geq 0$.

Proposition 7.0.3. Let $2 \leq q \leq \infty$. Suppose that $U(t)=\left[\rho, u, c_{1}, c_{2}\right]$ is the solution to the Cauchy problem (7.2)-(7.3) obtained in Proposition 7.0.1. Then the solution $U(t)=$ $\left[\rho, u, c_{1}, c_{2}\right]$ satisfies the following $L^{q}$-time decay estimates:

$$
\begin{gathered}
\|\rho\|_{L^{q}} \leq C(1+t)^{\frac{-3}{2}+\frac{3}{2 q}} \\
\|u\|_{L^{q}} \leq C(1+t)^{-\frac{3}{2}+\frac{1}{2 q}} \\
\left\|\left(c_{1}, c_{2}\right)\right\|_{L^{q}} \leq C(1+t)^{\frac{-3}{2}}
\end{gathered}
$$

for any $t \geq 0,2 \leq q \leq \infty$. 
The existence of global solutions in Theorem 6.0.1 is obtained directly from Proposition 7.0.1 and the derivation of rates in Theorem 6.0.1 is based on Proposition 7.0.3. 


\section{Chapter 8}

\section{Global solution of the nonlinear system $(7.2)$}

The goal of this chapter is to prove the global existence of solutions to the Cauchy problem (7.2) when initial data is a small, smooth perturbation near the steady-state $\left(n_{\infty}, 0,0,0\right)$. The proof is based on some uniform a priori estimates combined with the local existence that will be shown in subsections 8.0.1 and 8.0.2.

\subsubsection{Existence of local solutions}

The local existence of smooth solutions for symmetrizable hyperbolic equations $(7.2)_{1}$ and $(7.2)_{2}$ can be proved as in $[16,17]$. Since $(7.2)_{3}$ and $(7.2)_{4}$ are the heat equations, the local solutions exist. We construct a solution sequence $\left(\rho^{j}, u^{j}, c_{1}^{j}, c_{2}^{j}\right)_{j \geq 0}$ by iteratively solving the Cauchy problem on the following system

$$
\left\{\begin{array}{l}
\partial_{t} \rho^{j+1}+n_{\infty} \nabla \cdot u^{j}=-\nabla \cdot\left(\rho^{j+1} u^{j}\right) \\
\partial_{t} u^{j+1}+\nu u^{j+1}+\frac{p^{\prime}\left(n_{\infty}\right)}{n_{\infty}} \nabla \rho^{j+1}=-u^{j} \cdot \nabla u^{j+1}+\nabla c_{1}^{j}-\nabla c_{2}^{j} \\
-\left(\frac{p^{\prime}\left(\rho^{j}+n_{\infty}\right)}{\rho^{j}+n_{\infty}}-\frac{p^{\prime}\left(n_{\infty}\right)}{n_{\infty}}\right) \nabla \rho^{j+1} \\
\partial_{t} c_{1}^{j+1}-\Delta c_{1}^{j+1}+\left(a_{1}-a_{13} n_{\infty}\right) c_{1}^{j+1}=a_{11} c_{1}^{j^{2}}+a_{12} c_{2}^{j} c_{1}^{j+1}+a_{13} \rho^{j} c_{1}^{j+1} \\
\partial_{t} c_{2}^{j+1}-\Delta c_{2}^{j+1}+\left(a_{2}-a_{23} n_{\infty}\right) c_{2}^{j+1}=a_{21} c_{1}^{j} c_{2}^{j+1}+a_{22} c_{2}^{j^{2}}+a_{23} \rho^{j} c_{2}^{j+1}
\end{array}\right.
$$

with initial data

$$
\left.\left(\rho^{j+1}, u^{j+1}, c_{1}^{j+1}, c_{2}^{j+1}\right)\right|_{t=0}=\left(\rho_{0}, u_{0}, c_{1,0}, c_{2,0}\right),
$$


for $j \geq 0$, where $\left(\rho^{0}, u^{0}, c_{1}^{0}, c_{2}^{0}\right) \equiv(0,0,0,0)$ holds. For simplicity, in what follows, we write $U^{j}=\left(\rho^{j}, u^{j}, c_{1}^{j}, c_{2}^{j}\right)$ and $U_{0}=\left(\rho_{0}, u_{0}, c_{1,0}, c_{2,0}\right)$.

Lemma 8.0.1. There are constants $T_{1}>0, \epsilon_{0}>0, B>0$ such that if the initial data $U_{0} \in H^{N}\left(\mathbb{R}^{3}\right)$ and $\left\|U_{0}\right\|_{N} \leq \epsilon_{0}$, then for each $j \geq 0, U^{j} \in C\left(\left[0, T_{1}\right]: H^{N}\left(\mathbb{R}^{3}\right)\right)$ is well-defined and

$$
\sup _{0 \leq t \leq T_{1}}\left\|U^{j}(t)\right\|_{N} \leq B, \quad j \geq 0
$$

Moreover, $\left(U^{j}\right)_{j \geq 0}$ is a Cauchy sequence in Banach space $C\left(\left[0, T_{1}\right] ; H^{N}\left(\mathbb{R}^{3}\right)\right)$, and the limit function $U(x, t)$ of $\left(U^{j}\right)_{j \geq 0}$ satisfies

$$
\sup _{0 \leq t \leq T_{1}}\|U(t)\|_{N} \leq B
$$

and $U=\left(\rho, u, c_{1}, c_{2}\right)$ is a solution over $\left[0, T_{1}\right]$ to the Cauchy problem (7.2)-(7.3). Finally, the Cauchy problem (7.2)-(7.3) admits at most one solution $U \in C\left(\left[0, T_{1}\right]: H^{N}\left(\mathbb{R}^{3}\right)\right)$ satisfying (8.4).

\subsubsection{A Priori Estimates}

In this subsection, we provide some estimates for the solutions for any $t>0$. We establish the uniform-in-time a priori estimates for smooth solutions to Cauchy problem (7.2)-(7.3) by applying some basic energy estimates.

Lemma 8.0.2. (a priori estimates) Suppose that there exist a solution $U(t)=\left(\rho, u, c_{1}, c_{2}\right) \in$ $C\left([0, T] ; H^{N}\left(\mathbb{R}^{3}\right)\right.$ to the Cauchy problem (7.2)-(7.3), with

$$
\sup _{0 \leq t \leq T}\left\|\left(\rho, u, c_{1}, c_{2}\right)(t)\right\|_{N} \leq \epsilon
$$

for $0<\epsilon \leq 1$. Then, there are $\epsilon_{0}>0, C_{0}>0$ and $\lambda>0$ such that for any $\epsilon \leq \epsilon_{0}$,

$$
\mathcal{E}_{N}(U(t))+\lambda_{1} \int_{0}^{t} \mathcal{D}_{N}(U(t)) d s+\lambda_{2} \int_{0}^{t} \mathcal{D}_{N}^{h}(U(t)) d s \leq C_{0} \mathcal{E}_{N}\left(U_{0}\right)
$$

holds for any $t \in[0, T]$.

Proof. At first, we find the zero-order estimates. For the estimate of $\rho$, multiplying $\rho$ to both sides of the first equation of (7.2) and taking integrations in $x \in \mathbb{R}^{3}$, we obtain 


$$
\int_{\mathbb{R}^{3}} \rho \rho_{t} \mathrm{~d} x+n_{\infty} \int_{\mathbb{R}^{3}} \rho \nabla \cdot u \mathrm{~d} x=-\int_{\mathbb{R}^{3}} \rho \nabla \cdot(\rho u) \mathrm{d} x .
$$

Using integration by parts and the Cauchy-Schwarz inequality, we have

$$
\frac{1}{2} \int_{\mathbb{R}^{3}}\left(\rho^{2}\right)_{t} \mathrm{~d} x+n_{\infty} \int_{\mathbb{R}^{3}} \rho \nabla \cdot u \mathrm{~d} x \leq C\|\rho\|_{2} \int_{\mathbb{R}^{3}}|u|^{2}+|\nabla \rho|^{2} \mathrm{~d} x .
$$

For the estimate of $u$, multiplying $u$ to both sides of the second equation of (7.2) and taking integrations in $x \in \mathbb{R}^{3}$, we obtain

$$
\begin{aligned}
\int_{\mathbb{R}^{3}} u u_{t} \mathrm{~d} x+\int_{\mathbb{R}^{3}} u(u \cdot \nabla u) \mathrm{d} x-\nu & \int_{\mathbb{R}^{3}} u^{2} \mathrm{~d} x+\frac{p^{\prime}\left(n_{\infty}\right)}{n_{\infty}} \int_{\mathbb{R}^{3}} u \cdot \nabla \rho \mathrm{d} x=\int_{\mathbb{R}^{3}} u \nabla c_{1} \mathrm{~d} x \\
& -\int_{\mathbb{R}^{3}} u \nabla c_{2} \mathrm{~d} x-\int_{\mathbb{R}^{3}} u\left(\frac{p^{\prime}\left(\rho+n_{\infty}\right)}{\rho+n_{\infty}}-\frac{p^{\prime}\left(n_{\infty}\right)}{n_{\infty}}\right) \nabla \rho \mathrm{d} x .
\end{aligned}
$$

By using integration by parts and the Cauchy-Schwarz inequality, we have

$$
\begin{aligned}
\frac{1}{2} \int_{\mathbb{R}^{3}}\left(u^{2}\right)_{t} \mathrm{~d} x & +\nu \int_{\mathbb{R}^{3}}|u|^{2} \mathrm{~d} x-\frac{p^{\prime}\left(n_{\infty}\right)}{n_{\infty}} \int_{\mathbb{R}^{3}} \rho \nabla \cdot u \mathrm{~d} x \leq\|u\|_{3} \int_{\mathbb{R}^{3}}|u|^{2} \mathrm{~d} x \\
& +C \int_{\mathbb{R}^{3}}\left|\nabla c_{1}\right|^{2}+|u|^{2} \mathrm{~d} x+C \int_{\mathbb{R}^{3}}\left|\nabla c_{2}\right|^{2}+|u|^{2} \mathrm{~d} x \\
& +C\|\rho\|_{2} \int_{\mathbb{R}^{3}}|\nabla \rho|^{2}+|u|^{2} \mathrm{~d} x .
\end{aligned}
$$

For the estimates of $c_{1}$, we multiply $c_{1}$ to both sides of the equation of $c_{1}$ and integrate with respect to $x$, to get

$$
\begin{aligned}
\int_{\mathbb{R}^{3}} c_{1}\left(c_{1}\right)_{t} \mathrm{~d} x-\int_{\mathbb{R}^{3}} c_{1} \Delta c_{1} \mathrm{~d} x & +\left(a_{1}-a_{13} n_{\infty}\right) \int_{\mathbb{R}^{3}}\left|c_{1}\right|^{2} \mathrm{~d} x \leq a_{11} \sup _{x}\left|c_{1}\right| \int_{\mathbb{R}^{3}}\left|c_{1}\right|^{2} \mathrm{~d} x \\
& +a_{12} \sup _{x}\left|c_{2}\right| \int_{\mathbb{R}^{3}}\left|c_{1}\right|^{2} \mathrm{~d} x+a_{13} \sup _{x}|\rho| \int_{\mathbb{R}^{3}}\left|c_{1}\right|^{2} \mathrm{~d} x .
\end{aligned}
$$

By using integration by parts, we have

$$
\begin{array}{r}
\frac{1}{2} \int_{\mathbb{R}^{3}}\left(c_{1}^{2}\right)_{t} \mathrm{~d} x+\int_{\mathbb{R}^{3}}\left|\nabla c_{1}\right|^{2} \mathrm{~d} x+\left(a_{1}-a_{13} n_{\infty}\right) \int_{\mathbb{R}^{3}}\left|c_{1}\right|^{2} \mathrm{~d} x \leq a_{11}\left\|c_{1}\right\|_{2} \int_{\mathbb{R}^{3}}\left|c_{1}\right|^{2} \mathrm{~d} x \\
+a_{12}\left\|c_{2}\right\|_{2} \int_{\mathbb{R}^{3}}\left|c_{1}\right|^{2} \mathrm{~d} x+a_{13}\|\rho\|_{2} \int_{\mathbb{R}^{3}}\left|c_{1}\right|^{2} \mathrm{~d} x .
\end{array}
$$

Similarly, as above, from the equation of $c_{2}$, we have

$$
\begin{aligned}
& \frac{1}{2} \int_{\mathbb{R}^{3}}\left(c_{2}^{2}\right)_{t} \mathrm{~d} x+\int_{\mathbb{R}^{3}}\left|\nabla c_{2}\right|^{2} \mathrm{~d} x+\left(a_{2}-a_{23} n_{\infty}\right) \int_{\mathbb{R}^{3}}\left|c_{2}\right|^{2} \mathrm{~d} x \leq a_{21}\left\|c_{1}\right\|_{2} \int_{\mathbb{R}^{3}}\left|c_{2}\right|^{2} \mathrm{~d} x \\
& +a_{22}\left\|c_{2}\right\|_{2} \int_{\mathbb{R}^{3}}\left|c_{2}\right|^{2} \mathrm{~d} x+a_{23}\|\rho\|_{2} \int_{\mathbb{R}^{3}}\left|c_{2}\right|^{2} \mathrm{~d} x .
\end{aligned}
$$


By choosing the constant $d_{1}=\frac{p^{\prime}\left(n_{\infty}\right)}{n_{\infty}^{2}}$ and as long as $\mathcal{E}_{N}^{\frac{1}{2}}(U)$ is small so that

$$
\begin{aligned}
& \left(a_{1}-a_{13} n_{\infty}\right)>\left(a_{11}+a_{12}+a_{13}\right) \mathcal{E}_{N}^{\frac{1}{2}}(U), \\
& \left(a_{2}-a_{23} n_{\infty}\right)>\left(a_{21}+a_{22}+a_{23}\right) \mathcal{E}_{N}^{\frac{1}{2}}(U)
\end{aligned}
$$

are satisfied, we have

$$
\begin{aligned}
& \frac{1}{2} \frac{d}{d t} \int_{\mathbb{R}^{3}}\left(|u|^{2}+d_{1}|\rho|^{2}+\left|c_{1}\right|^{2}+\left|c_{2}\right|^{2}\right) \mathrm{d} x+\nu \int_{\mathbb{R}^{3}}|u|^{2} \mathrm{~d} x+\int_{\mathbb{R}^{3}}\left|\nabla c_{1}\right|^{2} \mathrm{~d} x+\int_{\mathbb{R}^{3}}\left|\nabla c_{2}\right|^{2} \mathrm{~d} x \\
& \quad+\left(a_{1}-a_{13} n_{\infty}\right) \int_{\mathbb{R}^{3}}\left|c_{1}\right|^{2} \mathrm{~d} x+\left(a_{2}-a_{23} n_{\infty}\right) \int_{\mathbb{R}^{3}}\left|c_{2}\right|^{2} \mathrm{~d} x \leq C\|\rho\|_{2} \int_{\mathbb{R}^{3}}|\nabla \rho|^{2} \mathrm{~d} x
\end{aligned}
$$

Now, we make estimates of the high-order derivatives of $\left(\rho, u, c_{1}, c_{2}\right)$. Take $\alpha$ with $1 \leq|\alpha| \leq$ $N$. Applying $\partial^{\alpha}$ to the second equation of (7.2), multiplying by $\partial^{\alpha} u$ and then integrating in $x$, we have

$$
\begin{aligned}
\frac{1}{2} \frac{d}{d t} \int_{\mathbb{R}^{3}}\left(\partial^{\alpha} u\right)^{2} \mathrm{~d} x & +\nu \int_{\mathbb{R}^{3}} \partial^{\alpha} u \partial^{\alpha} u \mathrm{~d} x+\int_{\mathbb{R}^{3}} \partial^{\alpha} u \partial^{\alpha}\left(\frac{p^{\prime}\left(\rho+n_{\infty}\right)}{\rho+n_{\infty}} \nabla \rho\right) \mathrm{d} x \\
& =-\int_{\mathbb{R}^{3}} \partial^{\alpha} u \partial^{\alpha}(u \cdot \nabla u) \mathrm{d} x+\int_{\mathbb{R}^{3}} \partial^{\alpha} u \partial^{\alpha} \nabla c_{1} \mathrm{~d} x-\int_{\mathbb{R}^{3}} \partial^{\alpha} u \partial^{\alpha} \nabla c_{2} \mathrm{~d} x .
\end{aligned}
$$

Thus

$$
\begin{aligned}
& \frac{1}{2} \frac{d}{d t} \int_{\mathbb{R}^{3}}\left(\partial^{\alpha} u\right)^{2} \mathrm{~d} x+\nu \int_{\mathbb{R}^{3}}\left|\partial^{\alpha} u\right|^{2} \mathrm{~d} x+\int_{\mathbb{R}^{3}} \partial^{\alpha} u \sum_{\beta=0}^{\alpha} C_{\alpha}^{\beta} \partial^{\beta}\left(\frac{p^{\prime}\left(\rho+n_{\infty}\right)}{\rho+n_{\infty}}\right) \partial^{\alpha-\beta} \nabla \rho \mathrm{d} x \\
& =-\int_{\mathbb{R}^{3}} \partial^{\alpha} u \sum_{\beta=0}^{\alpha} C_{\alpha}^{\beta}\left(\partial^{\alpha-\beta} u \cdot \nabla \partial^{\beta} u\right) \mathrm{d} x+\int_{\mathbb{R}^{3}} \partial^{\alpha} u \partial^{\alpha} \nabla c_{1} \mathrm{~d} x-\int_{\mathbb{R}^{3}} \partial^{\alpha} u \partial^{\alpha} \nabla c_{2} \mathrm{~d} x .
\end{aligned}
$$

We can estimate the third term on the left-hand side of the previous equality for $\beta=0$ by using integration by parts and using the first equation, to give

$$
\begin{aligned}
& \int_{\mathbb{R}^{3}} \partial^{\alpha} u\left(\frac{p^{\prime}\left(\rho+n_{\infty}\right)}{\rho+n_{\infty}}\right) \partial^{\alpha} \nabla \rho \mathrm{d} x=-\int_{\mathbb{R}^{3}} \partial^{\alpha} \nabla \cdot u\left(\frac{p^{\prime}\left(\rho+n_{\infty}\right)}{\rho+n_{\infty}}\right) \partial^{\alpha} \rho \mathrm{d} x-\int_{\mathbb{R}^{3}} \partial^{\alpha} u \nabla\left(\frac{p^{\prime}\left(\rho+n_{\infty}\right)}{\rho+n_{\infty}}\right) \partial^{\alpha} \rho \mathrm{d} x \\
& \left.=\int_{\mathbb{R}^{3}} \partial^{\alpha}\left[\frac{1}{\rho+n_{\infty}} \rho_{t}+\frac{\nabla\left(\rho+n_{\infty}\right) \cdot u}{\rho+n_{\infty}}\right)\right] \frac{p^{\prime}\left(\rho+n_{\infty}\right)}{\rho+n_{\infty}} \partial^{\alpha} \rho \mathrm{d} x-\int_{\mathbb{R}^{3}} \partial^{\alpha} u \nabla\left(\frac{p^{\prime}\left(\rho+n_{\infty}\right)}{\rho+n_{\infty}}\right) \partial^{\alpha} \rho \mathrm{d} x \\
& =\frac{1}{2} \int_{\mathbb{R}^{3}} \frac{p^{\prime}\left(\rho+n_{\infty}\right)}{\left(\rho+n_{\infty}\right)^{2}}\left(\partial^{\alpha} \rho^{2}\right)_{t} \mathrm{~d} x+\int_{\mathbb{R}^{3}} \frac{p^{\prime}\left(\rho+n_{\infty}\right)}{\left(\rho+n_{\infty}\right)} \partial^{\alpha}\left(\frac{1}{\rho+n_{\infty}}\right) \rho_{t} \partial^{\alpha} \rho \mathrm{d} x \\
& +\int_{\mathbb{R}^{3}} \frac{1}{\rho+n_{\infty}} \partial^{\alpha}(\nabla \rho \cdot u) \frac{p^{\prime}\left(\rho+n_{\infty}\right)}{\left(\rho+n_{\infty}\right)} \partial^{\alpha} \rho \mathrm{d} x+\int_{\mathbb{R}^{3}} \partial^{\alpha}\left(\frac{1}{\rho+n_{\infty}}\right)(\nabla \rho \cdot u) \frac{p^{\prime}\left(\rho+n_{\infty}\right)}{\left(\rho+n_{\infty}\right)} \partial^{\alpha} \rho \mathrm{d} x \\
& -\int_{\mathbb{R}^{3}} \partial^{\alpha} u \nabla\left(\frac{p^{\prime}\left(\rho+n_{\infty}\right)}{\rho+n_{\infty}}\right) \partial^{\alpha} \rho \mathrm{d} x .
\end{aligned}
$$


For $0<\beta \leq \alpha$ the third term on the left-hand side after being moved to the right-hand side of (8.12) will be bounded by

$$
C\|\rho\|_{N} \int_{\mathbb{R}^{3}} \partial^{\alpha} u \partial^{\alpha} \rho \mathrm{d} x .
$$

Then, the terms on the right-hand side of (8.12) are bounded by

$$
\begin{aligned}
& C\|\rho\|_{N} \int_{\mathbb{R}^{3}}\left(\left|\partial^{\alpha} u\right|^{2}+\left|\partial^{\alpha} \rho\right|^{2}\right) \mathrm{d} x+C\|u\|_{N} \int_{\mathbb{R}^{3}}\left|\partial^{\alpha} u\right|^{2} \mathrm{~d} x \\
& +C \int_{\mathbb{R}^{3}}\left(\left|\partial^{\alpha} u\right|^{2}+\left|\partial^{\alpha} \nabla c_{1}\right|^{2}\right) \mathrm{d} x+C \int_{\mathbb{R}^{3}}\left(\left|\partial^{\alpha} u\right|^{2}+\left|\partial^{\alpha} \nabla c_{2}\right|^{2}\right) \mathrm{d} x .
\end{aligned}
$$

Plugging (8.13)-(8.15) into (8.12), integrating with respect to $t$, and using the CauchySchwarz inequality, we get

$$
\begin{aligned}
\frac{1}{2}\left\|\partial^{\alpha} u\right\|^{2} & +C_{1}\left\|\partial^{\alpha} \rho\right\|^{2}+\nu \int_{0}^{t}\left\|\partial^{\alpha} u\right\|^{2} \mathrm{~d} s \\
& \leq C\left\|\partial^{\alpha} u_{0}\right\|+C\left\|\partial^{\alpha} \rho_{0}\right\|+C\|\rho\|_{N} \int_{0}^{t}\left(\left\|\partial^{\alpha} u\right\|^{2}+\left\|\partial^{\alpha} \rho\right\|^{2}\right) \mathrm{d} s+C\|u\|_{N} \int_{0}^{t}\left\|\partial^{\alpha} \rho\right\|^{2} \mathrm{~d} s \\
& +C\|u\|_{N} \int_{0}^{t}\left\|\partial^{\alpha} u\right\|^{2} \mathrm{~d} s+C \int_{0}^{t}\left(\left\|\partial^{\alpha} u\right\|^{2}+\left\|\partial^{\alpha} \nabla c_{1}\right\|^{2}\right) \mathrm{d} s \\
& +C \int_{0}^{t}\left(\left\|\partial^{\alpha} u\right\|^{2}+\left\|\partial^{\alpha} \nabla c_{2}\right\|^{2}\right) \mathrm{d} s
\end{aligned}
$$

In a similar way as above, we estimate $c_{1}$ and $c_{2}$ as follows:

$$
\begin{aligned}
\frac{1}{2}\left\|\partial^{\alpha} c_{1}\right\|^{2} & +\int_{0}^{t}\left\|\nabla \partial^{\alpha} c_{1}\right\|^{2} \mathrm{~d} s+\left(a_{12}-n_{\infty} a_{11}\right) \int_{0}^{t}\left\|\partial^{\alpha} c_{1}\right\|^{2} \mathrm{~d} s \leq C\left\|\partial^{\alpha} c_{1,0}\right\| \\
& +C\|\rho\|_{N} \int_{0}^{t}\left\|\partial^{\alpha} c_{1}\right\|^{2} \mathrm{~d} s+C\left\|c_{1}\right\|_{N} \int_{0}^{t}\left(\left\|\partial^{\alpha} c_{1}\right\|^{2}+\left\|\partial^{\alpha} \rho\right\|^{2}\right) \mathrm{d} s \\
& +C\left\|c_{2}\right\|_{N} \int_{0}^{t}\left\|\partial^{\alpha} c_{1}\right\|^{2} \mathrm{~d} s+C\left\|c_{1}\right\|_{N} \int_{0}^{t}\left(\left\|\partial^{\alpha} c_{1}\right\|^{2}+\left\|\partial^{\alpha} c_{2}\right\|^{2}\right) \mathrm{d} s
\end{aligned}
$$

and

$$
\begin{aligned}
\frac{1}{2}\left\|\partial^{\alpha} c_{2}\right\|^{2}+ & \int_{0}^{t}\left\|\nabla \partial^{\alpha} c_{2}\right\|^{2} \mathrm{~d} s+\left(a_{22}-n_{\infty} a_{21}\right) \int_{0}^{t}\left\|\partial^{\alpha} c_{2}\right\|^{2} \mathrm{~d} s \leq C\left\|\partial^{\alpha} c_{2,0}\right\| \\
& +C\|\rho\|_{N} \int_{0}^{t}\left(\left\|\partial^{\alpha} c_{2}\right\|^{2} \mathrm{~d} s+C\left\|c_{2}\right\|_{N} \int_{0}^{t}\left(\left\|\partial^{\alpha} c_{2}\right\|^{2}+\left\|\partial^{\alpha} \rho\right\|^{2}\right) \mathrm{d} s\right. \\
& +C\left\|c_{1}\right\|_{N} \int_{0}^{t}\left(\left\|\partial^{\alpha} c_{2}\right\|^{2} \mathrm{~d} s+C\left\|c_{2}\right\|_{N} \int_{0}^{t}\left(\left\|\partial^{\alpha} c_{2}\right\|^{2}+\left\|\partial^{\alpha} c_{1}\right\|^{2}\right) \mathrm{d} s .\right.
\end{aligned}
$$


Then, by taking the summation of (8.16)-(8.18) over $|\alpha| \leq N$, we have

$$
\begin{aligned}
\frac{1}{2}\left(\|u\|_{N}^{2}\right. & \left.+C_{1}\|\rho\|_{N}^{2}+\left\|c_{1}\right\|_{N}^{2}+\left\|c_{2}\right\|_{N}^{2}\right)+\nu \int_{0}^{t}\|u\|_{N}^{2} \mathrm{~d} s+\int_{0}^{t}\left\|\nabla c_{1}\right\|_{N}^{2} \mathrm{~d} s+\int_{0}^{t}\left\|\nabla c_{2}\right\|_{N}^{2} \mathrm{~d} s \\
& +\left(a_{1}-n_{\infty} a_{13}\right) \int_{0}^{t}\left\|c_{1}\right\|_{N}^{2} \mathrm{~d} s+\left(a_{2}-n_{\infty} a_{23}\right) \int_{0}^{t}\left\|c_{2}\right\|_{N}^{2} \mathrm{~d} s \\
& \leq C_{0}\left\|U_{0}\right\|_{N}+C\|\rho\|_{N} \int_{0}^{t}\left(\|u\|_{N}^{2}+\|\rho\|_{N}^{2}+\left\|c_{1}\right\|_{N}^{2}+\left\|c_{2}\right\|_{N}^{2}\right) \mathrm{d} s+C\left\|u, c_{1}, c_{2}\right\|_{N} \int_{0}^{t}\|\rho\|_{N}^{2} \mathrm{~d} s \\
& +C\|u\|_{N} \int_{0}^{t}\|u\|_{N}^{2} \mathrm{~d} s+C \int_{0}^{t}\left(\|u\|_{N}^{2}+\left\|c_{1}\right\|_{N}^{2}+\left\|c_{2}\right\|_{N}^{2}\right) \mathrm{d} s \\
& +C\left\|c_{1}\right\|_{N} \int_{0}^{t}\left(\left\|c_{1}\right\|_{N}^{2}+\left\|c_{2}\right\|_{N}^{2}\right) \mathrm{d} s+C\left\|c_{2}\right\|_{N} \int_{0}^{t}\left(\left\|c_{1}\right\|_{N}^{2}+\left\|c_{2}\right\|_{N}^{2}\right) \mathrm{d} s .
\end{aligned}
$$

Let $|\alpha| \leq N-1$. Applying $\partial^{\alpha}$ to $(7.2)_{2}$, multiplying it by $\partial^{\alpha} \nabla \rho$ and taking integrations in $x$ gives

$$
\begin{aligned}
\int_{\mathbb{R}^{3}} \partial^{\alpha} \nabla \rho \partial^{\alpha} u_{t} \mathrm{~d} x & +\nu \int_{\mathbb{R}^{3}} \partial^{\alpha} \nabla \rho \partial^{\alpha} u \mathrm{~d} x+\frac{p^{\prime}\left(n_{\infty}\right)}{n_{\infty}} \int_{\mathbb{R}^{3}} \partial^{\alpha} \nabla \rho \partial^{\alpha} \nabla \rho \mathrm{d} x \\
& =-\int_{\mathbb{R}^{3}} \partial^{\alpha} \nabla \rho \partial^{\alpha}(u \cdot \nabla u) \mathrm{d} x+\int_{\mathbb{R}^{3}} \partial^{\alpha} \nabla \rho \partial^{\alpha} \nabla c_{1} \mathrm{~d} x-\int_{\mathbb{R}^{3}} \partial^{\alpha} \nabla \rho \partial^{\alpha} \nabla c_{2} \mathrm{~d} x \\
& \left.-\int_{\mathbb{R}^{3}} \partial^{\alpha} \nabla \rho \partial^{\alpha}\left(\frac{p^{\prime}\left(\rho+n_{\infty}\right)}{\rho+n_{\infty}}-\frac{p^{\prime}\left(n_{\infty}\right)}{n_{\infty}}\right) \nabla \rho\right) \mathrm{d} x,
\end{aligned}
$$

which further, by replacing $\partial_{t} \rho$ from the first equation of (7.2) and then using integration by parts, implies

$$
\begin{aligned}
\int_{\mathbb{R}^{3}}\left(\partial^{\alpha} \nabla \rho \partial^{\alpha} u\right)_{t} \mathrm{~d} x & +\frac{p^{\prime}\left(n_{\infty}\right)}{n_{\infty}} \int_{\mathbb{R}^{3}}\left|\partial^{\alpha} \nabla \rho\right|^{2} \mathrm{~d} x \\
& =-\nu \int_{\mathbb{R}^{3}} \partial^{\alpha} \nabla \rho \partial^{\alpha} u \mathrm{~d} x-\int_{\mathbb{R}^{3}} \partial^{\alpha} \nabla \rho \partial^{\alpha}(u \cdot \nabla u) \mathrm{d} x \\
& +\int_{\mathbb{R}^{3}} \partial^{\alpha} \nabla \rho \partial^{\alpha} \nabla c_{1} \mathrm{~d} x-\int_{\mathbb{R}^{3}} \partial^{\alpha} \nabla \rho \partial^{\alpha} \nabla c_{2} \mathrm{~d} x \\
& \left.-\int_{\mathbb{R}^{3}} \partial^{\alpha} \nabla \rho \partial^{\alpha}\left(\frac{p^{\prime}\left(\rho+n_{\infty}\right)}{\rho+n_{\infty}}-\frac{p^{\prime}\left(n_{\infty}\right)}{n_{\infty}}\right) \nabla \rho\right) \mathrm{d} x \\
& -\int_{\mathbb{R}^{3}} \partial^{\alpha} \nabla \cdot u \partial^{\alpha} \nabla \cdot\left(\left(\rho+n_{\infty}\right) u\right) \mathrm{d} x .
\end{aligned}
$$

Applying the Cauchy-Schwarz inequality we obtain

$$
\begin{aligned}
\frac{d}{d t} \int_{\mathbb{R}^{3}}\left(\partial^{\alpha} \nabla \rho \partial^{\alpha} u\right) \mathrm{d} x & +\lambda_{2}\left\|\partial^{\alpha} \nabla \rho\right\|^{2} \\
& \left.\leq C\left(\left\|\nabla \cdot \partial^{\alpha} u\right\|^{2}+\left\|\partial^{\alpha} u\right\|^{2}\right)+C\left\|\partial^{\alpha} \nabla\left[c_{1}, c_{2}\right]\right\|^{2}\right) \\
& +C\left(\|[\rho, u]\|_{N}\left\|\nabla \cdot \partial^{\alpha}[\rho, u]\right\|^{2}\right) .
\end{aligned}
$$


Then, after taking summation over $|\alpha| \leq N-1$ and integrating with respect to $t$, we obtain

$$
\begin{aligned}
\sum_{|\alpha| \leq N-1} \int_{\mathbb{R}^{3}} \partial^{\alpha} \nabla \rho \partial^{\alpha} u \mathrm{~d} x & +\lambda_{2} \int_{0}^{t}\|\nabla \rho\|_{N-1}^{2} \mathrm{~d} s \leq\left.\sum_{|\alpha| \leq N-1} \int_{\mathbb{R}^{3}} \partial^{\alpha} \nabla \rho \partial^{\alpha} u \mathrm{~d} x\right|_{t=0} \\
& +C \int_{0}^{t}\|u\|_{N}^{2} \mathrm{~d} s+C \int_{0}^{t}\left\|\nabla\left[c_{1}, c_{2}\right]\right\|_{N-1}^{2} \mathrm{~d} s \\
& +C\|[\rho, u]\|_{N} \int_{0}^{t}\|\nabla \cdot[\rho, u]\|_{N-1}^{2} \mathrm{~d} s
\end{aligned}
$$

By taking a linear combination $(8.11)+(8.19)+k(8.20)$, we have

$$
\begin{aligned}
\|U\|_{N}^{2}+ & k \sum_{|\alpha| \leq N-1} \int_{\mathbb{R}^{3}} \partial^{\alpha} \nabla \rho \partial^{\alpha} u \mathrm{~d} x \\
& +\lambda_{1} \int_{0}^{t}\left\|\nabla c_{1}, c_{2}\right\|_{N}^{2} \mathrm{~d} s+\lambda_{2} \int_{0}^{t}\left(\|\nabla \rho\|_{N-1}^{2}+\left\|u, c_{1}, c_{2}\right\|_{N}^{2}\right) \mathrm{d} s \leq C_{0}\left\|U_{0}\right\|_{N}^{2}
\end{aligned}
$$

for constant $0<k \ll 1$. Then

$$
\|U\|_{N}^{2}+k \sum_{|\alpha| \leq N-1} \int_{0}^{t} \int_{\mathbb{R}^{3}} \partial^{\alpha} \nabla \rho \partial^{\alpha} u \mathrm{~d} x \mathrm{~d} s \sim\|U\|_{N}^{2} .
$$

This completes the proof of Lemma 8.0.2.

Based on the argument in Lemma 8.0.1 and Lemma 8.0.2, now we start to prove Proposition 7.0.1.

Proof of Proposition 7.0.1. Choose a positive constant $\bar{\epsilon}=\min \left\{\epsilon_{0}, \epsilon_{1}\right\}$,

where $\epsilon_{0}>0$ and $\epsilon_{1}>0$ are given in Lemma 8.0.1 and Lemma 8.0.2. Let $U_{0} \in H^{N}\left(\mathbb{R}^{3}\right)$ satisfy

$$
\left\|U_{0}\right\|_{H^{N}} \leq \frac{\bar{\epsilon}}{2 \sqrt{C_{0}+1}}
$$

Now, let us define

$$
T=\left\{t \geq 0: \sup _{0 \leq s \leq t}\|U(s)\|_{H^{N}} \leq \bar{\epsilon}\right\}
$$

Note that

$$
\left\|U_{0}\right\|_{H^{N}} \leq \frac{\bar{\epsilon}}{2 \sqrt{C_{0}+1}} \leq \frac{\bar{\epsilon}}{2}<\bar{\epsilon} \leq \epsilon_{0}
$$

Then $T>0$ holds from the local existence result. If $T$ is finite, from the definition of $T$, we have

$$
\sup _{0 \leq s \leq t}\|U\|_{H^{N}}=\bar{\epsilon}
$$


On the other hand, by Lemma 8.0.2 we have

$$
\sup _{0 \leq s \leq t}\|U(s)\|_{H^{N}} \leq \sqrt{C_{0}}\left\|U_{0}\right\|_{H^{N}} \leq \frac{\bar{\epsilon} \sqrt{C_{0}}}{2 \sqrt{C_{0}+1}} \leq \frac{\bar{\epsilon}}{2}
$$

which is a contradiction to 8.22. Then $T=\infty$ holds true. This implies that local solution $U(t)$ obtained in Lemma 8.0.1 can be extended to infinity in time. Thus, we have a global solution $\left(\rho, u, c_{1}, c_{2}\right)(t) \in C\left([0, \infty) ; H^{N}\right)$. This completes the proof of Proposition 7.0.1. 


\section{Chapter 9}

\section{Linearized Homogeneous System}

In this chapter, to study the time-decay property of solutions to the nonlinear system (7.2), we have to consider the following Cauchy problem for the corresponding linearized equations around the constant state $\left[n_{\infty}, 0,0,0\right]$. Then $U=\left[\rho, u, c_{1}, c_{2}\right]$ satisfies

$$
\left\{\begin{array}{l}
\partial_{t} \rho+n_{\infty} \nabla \cdot u=g_{1} \\
\partial_{t} u+\nu u+\frac{p^{\prime}\left(n_{\infty}\right)}{n_{\infty}} \nabla \rho+\nabla c_{1}-\nabla c_{2}=g_{2} \\
\partial_{t} c_{1}-\Delta c_{1}+\left(a_{1}+n_{\infty} a_{13}\right) c_{1}=g_{3} \\
\partial_{t} c_{2}-\Delta c_{2}+\left(a_{2}+n_{\infty} a_{23}\right) c_{2}=g_{4},
\end{array}\right.
$$

with initial data

$$
\left.\left(\rho, u, c_{1}, c_{2}\right)\right|_{t=0}=\left(\rho_{0}, u_{0}, c_{1,0}, c_{2,0}\right)
$$

Here the nonlinear source term takes the form

$$
\left\{\begin{array}{l}
g_{1}=-\nabla \cdot(\rho u) \\
g_{2}=-u \cdot \nabla u-\left(\frac{p^{\prime}\left(\rho+n_{\infty}\right)}{\rho+n_{\infty}}-\frac{p^{\prime}\left(n_{\infty}\right)}{n_{\infty}}\right) \nabla \rho \\
g_{3}=a_{11} c_{1}^{2}+a_{12} c_{1} c_{2}+a_{13} c_{1} \rho \\
g_{4}=a_{21} c_{1} c_{2}+a_{22} c_{2}^{2}+a_{23} c_{2} \rho .
\end{array}\right.
$$

To obtain the time-decay rates of the solution to the system (9.1) in the next chapter, we are concerned with the following Cauchy problem for the linearized homogenous system corresponding with the system (9.1): 


$$
\left\{\begin{array}{l}
\partial_{t} \rho+n_{\infty} \nabla \cdot u=0 \\
\partial_{t} u+\nu u+\frac{p^{\prime}\left(n_{\infty}\right)}{n_{\infty}} \nabla \rho-\nabla c_{1}+\nabla c_{2}=0 \\
\partial_{t} c_{1}-\Delta c_{1}+\left(a_{1}-n_{\infty} a_{13}\right) c_{1}=0 \\
\partial_{t} c_{2}-\Delta c_{2}+\left(a_{2}-n_{\infty} a_{23}\right) c_{2}=0
\end{array}\right.
$$

with initial data

$$
\left.\left(\rho, u, c_{1}, c_{2}\right)\right|_{t=0}=U_{0}=\left(\rho_{0}, u_{0}, c_{1,0}, c_{2,0}\right)
$$

In this chapter, we let $U=\left[\rho, u, c_{1}, c_{2}\right]$ be the solution to the system (9.4).

\subsubsection{Representation of solutions}

In this subsection, we find the explicit representation of the Fourier transform of the solution $U=e^{B t} U_{0}$ to the Cauchy problem (9.4)-(9.5), where $e^{t B}$ is the linear solution operator.

After taking the Fourier transform in $x$ for the first equation of (9.4), we have

$$
\hat{\rho}_{t}+n_{\infty} i \xi \hat{u}=0
$$

with initial data $\left.\hat{\rho}\right|_{t=0}=\hat{\rho}_{0}$.

Similarly, by taking the Fourier transform for the second equation of (9.4), we get

$$
\hat{u}_{t}+\nu \hat{u}+\frac{p^{\prime}\left(n_{\infty}\right)}{n_{\infty}} i \xi \hat{\rho}-i \xi \hat{c}_{1}+i \xi \hat{c}_{2}=0
$$

with initial data $\left.\hat{u}\right|_{t=0}=\hat{u}_{0}$.

Further, by taking the dot product of (9.7) with $\hat{\xi}$, we have

$$
\tilde{\xi} \cdot \hat{u}_{t}+\nu \tilde{\xi} \cdot \hat{u}+i \frac{p^{\prime}\left(n_{\infty}\right)}{n_{\infty}} \tilde{\xi} \cdot \xi \hat{\rho}-i \tilde{\xi} \cdot \xi \hat{c}_{1}+i \tilde{\xi} \cdot \xi \hat{c}_{2}=0
$$

Here and in the sequel, we set $\tilde{\xi}=\frac{\xi}{|\xi|}$ for $|\xi| \neq 0$.

Similarly for $\left[c_{1}, c_{2}\right]$, by taking the Fourier transform for the third and fourth equations of (9.4), we get

$$
\begin{aligned}
& \partial_{t} \hat{c}_{1}+|\xi|^{2} \hat{c}_{1}+\left(a_{1}-a_{13} n_{\infty}\right) \hat{c}_{1}=0 \\
& \partial_{t} \hat{c}_{2}+|\xi|^{2} \hat{c}_{2}+\left(a_{2}-a_{23} n_{\infty}\right) \hat{c}_{2}=0 .
\end{aligned}
$$


Then, we have

$$
\left\{\begin{array}{l}
\hat{\rho}_{t}+i n_{\infty} \xi \cdot \hat{u}=0 \\
\tilde{\xi} \cdot \hat{u}_{t}+\nu \tilde{\xi} \cdot \hat{u}+i \frac{p^{\prime}\left(n_{\infty}\right)}{n_{\infty}} \tilde{\xi} \cdot \xi \hat{\rho}-i \tilde{\xi} \cdot \xi \hat{c}_{1}+i \tilde{\xi} \cdot \xi \hat{c}_{2}=0 \\
\partial_{t} \hat{c}_{1}+|\xi|^{2} \hat{c}_{1}+\left(a_{1}-a_{13} n_{\infty}\right) \hat{c}_{1}=0 \\
\partial_{t} \hat{c}_{2}+|\xi|^{2} \hat{c}_{2}+\left(a_{2}-a_{23} n_{\infty}\right) \hat{c}_{2}=0 .
\end{array}\right.
$$

We can rewrite (9.9) as

$$
\partial_{t} \hat{U}=A(\xi) \hat{U}
$$

with $\hat{U}(t, \xi)=\left(\hat{\rho}(t, \xi), \tilde{\xi} \cdot \hat{u}(t, \xi), \hat{c}_{1}(t, \xi), \hat{c}_{2}(t, \xi)\right)^{T}$ and

$$
A(\xi)=\left[\begin{array}{cccc}
0 & -i n_{\infty}|\xi| & 0 & 0 \\
-i \frac{p^{\prime}\left(n_{\infty}\right)}{n_{\infty}} \frac{\xi^{2}}{|\xi|} & -\nu & i|\xi| & -i|\xi| \\
0 & 0 & -|\xi|^{2}-\left(a_{1}-a_{13} n_{\infty}\right) & 0 \\
0 & 0 & 0 & -|\xi|^{2}-\left(a_{2}-a_{23} n_{\infty}\right)
\end{array}\right]
$$

where $T$ denotes the transpose of a row vector. Then, the eigenvalues of the system are as follows

$$
\begin{gathered}
\lambda_{1}=-\frac{1}{2} \nu+\frac{1}{2} \sqrt{\nu^{2}-4 p^{\prime}\left(n_{\infty}\right)|\xi|^{2}} \\
\lambda_{2}=-\frac{1}{2} \nu-\frac{1}{2} \sqrt{\nu^{2}-4 p^{\prime}\left(n_{\infty}\right)|\xi|^{2}} \\
\lambda_{3}=-|\xi|^{2}-\left(a_{1}-a_{13} n_{\infty}\right) \\
\lambda_{4}=-|\xi|^{2}-\left(a_{2}-a_{23} n_{\infty}\right) .
\end{gathered}
$$

Therefore, the eigenvectors corresponding to the eigenvalues $\lambda$ of $A(\xi)$ that satisfy $(A-$ $\lambda I) X=0$ are

$$
v_{1}=\left[\begin{array}{c}
i n_{\infty}|\xi| \\
-\lambda_{1} \\
0 \\
0
\end{array}\right]
$$


and

$$
\begin{aligned}
& v_{2}=\left[\begin{array}{c}
i n_{\infty}|\xi| \\
-\lambda_{2} \\
0 \\
0
\end{array}\right] \\
& v_{3}=\left[\begin{array}{c}
\frac{n_{\infty}|\xi|^{2}}{\lambda_{3}} \\
i|\xi| \\
\frac{p^{\prime}\left(n_{\infty}\right)|\xi|^{2}}{\lambda_{3}}+\left(\nu+\lambda_{3}\right) \\
0
\end{array}\right] \\
& v_{4}=\left[\begin{array}{c}
-\frac{n_{\infty}|\xi|^{2}}{\lambda_{4}} \\
-i|\xi| \\
0 \\
\frac{p^{\prime}\left(n_{\infty}\right)|\xi|^{2}}{\lambda_{4}}+\left(\nu+\lambda_{4}\right)
\end{array}\right]
\end{aligned}
$$

From above, one can define the general solution of (9.9) as

$$
\left[\begin{array}{c}
\hat{\rho} \\
\tilde{\xi} \cdot \hat{u} \\
\hat{c}_{1} \\
\hat{c}_{2}
\end{array}\right]=\left[\begin{array}{cccc}
i n_{\infty}|\xi| e^{\lambda_{1} t} & i n_{\infty}|\xi| e^{\lambda_{2} t} & \frac{n_{\infty}|\xi|^{2}}{\lambda_{3}} e^{\lambda_{3} t} & -\frac{n_{\infty}|\xi|^{2}}{\lambda_{4}} e^{\lambda_{4} t} \\
-\lambda_{1} e^{\lambda_{1} t} & -\lambda_{2} e^{\lambda_{2} t} & i|\xi| e^{\lambda_{3} t} & -i|\xi| e^{\lambda_{4} t} \\
0 & 0 & \frac{p^{\prime}\left(n_{\infty}\right)|\xi|^{2}}{\lambda_{3}}+\left(\nu+\lambda_{3}\right) e^{\lambda_{3} t} & 0 \\
0 & 0 & 0 & \frac{p^{\prime}\left(n_{\infty}\right)|\xi|^{2}}{\lambda_{4}}+\left(\nu+\lambda_{4}\right) e^{\lambda_{4} t}
\end{array}\right]\left[\begin{array}{c}
d_{1} \\
d_{2} \\
d_{3} \\
d_{4}
\end{array}\right],
$$

where $d_{1}, d_{2}, d_{3}, d_{4}$ satisfy

$$
\left[\begin{array}{c}
\left.\hat{\rho}\right|_{t=0} \\
\left.\tilde{\xi} \cdot \hat{u}\right|_{t=0} \\
\left.\hat{c}_{1}\right|_{t=0} \\
\left.\hat{c}_{2}\right|_{t=0}
\end{array}\right]=\left[\begin{array}{cccc}
\operatorname{in}_{\infty}|\xi| & \operatorname{in}_{\infty}|\xi| & \frac{n_{\infty}|\xi|^{2}}{\lambda_{3}} & -\frac{n_{\infty}|\xi|^{2}}{\lambda_{4}} \\
-\lambda_{1} & -\lambda_{2} & i|\xi| & -i|\xi| \\
0 & 0 & \frac{p^{\prime}\left(n_{\infty}\right)|\xi|^{2}}{\lambda_{3}}+\left(\nu+\lambda_{3}\right) & 0 \\
0 & 0 & 0 & \frac{p^{\prime}\left(n_{\infty}\right)|\xi|^{2}}{\lambda_{4}}+\left(\nu+\lambda_{4}\right)
\end{array}\right]\left[\begin{array}{c}
d_{1} \\
d_{2} \\
d_{3} \\
d_{4}
\end{array}\right] .
$$

From this we deduce that 


$$
\left[\begin{array}{l}
d_{1} \\
d_{2} \\
d_{3} \\
d_{4}
\end{array}\right]=\frac{1}{i n_{\infty}|\xi|\left(\lambda_{1}-\lambda_{2}\right)}\left[\begin{array}{cccc}
-\lambda_{2} & -i n_{\infty}|\xi| & \frac{n_{\infty}|\xi|^{2}\left(\frac{\lambda_{2}}{\lambda_{3}}-1\right)}{a_{3}} & -\frac{n_{\infty}|\xi|^{2}\left(\frac{\lambda_{2}}{\lambda_{4}}-1\right)}{a_{4}} \\
\lambda_{1} & i n_{\infty}|\xi| & -\frac{n_{\infty}|\xi|^{2}\left(\frac{\lambda_{1}}{\lambda_{3}}-1\right)}{a_{3}} & \frac{n_{\infty}|\xi|^{2}\left(\frac{\lambda_{1}}{\lambda_{4}}-1\right)}{a_{4}} \\
0 & 0 & \frac{i n_{\infty}|\xi|\left(\lambda_{1}-\lambda_{2}\right)}{a_{3}} & 0 \\
0 & 0 & 0 & \frac{i n_{\infty}|\xi|\left(\lambda_{1}-\lambda_{2}\right)}{a_{4}}
\end{array}\right]\left[\begin{array}{c}
\hat{\rho}_{0} \\
\tilde{\xi} \cdot \hat{u}_{0} \\
\hat{c}_{1,0} \\
\hat{c}_{2,0}
\end{array}\right]
$$

where $a_{3}=\frac{p^{\prime}\left(n_{\infty}\right)|\xi|^{2}}{\lambda_{3}}+\left(\nu+\lambda_{3}\right)$ and $a_{4}=\frac{p^{\prime}\left(n_{\infty}\right)|\xi|^{2}}{\lambda_{4}}+\left(\nu+\lambda_{4}\right)$.

It is straightforward to obtain

$$
\begin{aligned}
& \hat{\rho}=\frac{\lambda_{1} e^{\lambda_{2} t}-\lambda_{2} e^{\lambda_{1} t}}{\lambda_{1}-\lambda_{2}} \hat{\rho}_{0}-i n_{\infty} \frac{e^{\lambda_{1} t}-e^{\lambda_{2} t}}{\lambda_{1}-\lambda_{2}} \xi \cdot \hat{u}_{0} \\
& +\frac{n_{\infty}|\xi|^{2}\left(\frac{\lambda_{2}}{\lambda_{3}}-1\right) e^{\lambda_{1} t}-n_{\infty}|\xi|^{2}\left(\frac{\lambda_{1}}{\lambda_{3}}-1\right) e^{\lambda_{2} t}+\frac{n_{\infty}|\xi|^{2}}{\lambda_{3}}\left(\lambda_{1}-\lambda_{2}\right) e^{\lambda_{3} t}}{a_{3}\left(\lambda_{1}-\lambda_{2}\right)} \hat{c}_{1,0} \\
& +\frac{n_{\infty}|\xi|^{2}\left(1-\frac{\lambda_{2}}{\lambda_{4}}\right) e^{\lambda_{1} t}-n_{\infty}|\xi|^{2}\left(\frac{\lambda_{1}}{\lambda_{4}}-1\right) e^{\lambda_{2} t}+\frac{n_{\infty}|\xi|^{2}}{\lambda_{4}}\left(\lambda_{1}-\lambda_{2}\right) e^{\lambda_{4} t}}{a_{4}\left(\lambda_{1}-\lambda_{2}\right)} \hat{c}_{2,0} \\
& \tilde{\xi} \cdot \hat{u}=\frac{\lambda_{1} \lambda_{2}}{i n_{\infty}|\xi|}\left(\frac{e^{\lambda_{1} t}-e^{\lambda_{2} t}}{\lambda_{1}-\lambda_{2}}\right) \hat{\rho}_{0}+\left(\frac{\lambda_{1} e^{\lambda_{1} t}-\lambda_{2} e^{\lambda_{2} t}}{\lambda_{1}-\lambda_{2}}\right) \tilde{\xi} \cdot \hat{u}_{0} \\
& +\frac{i \lambda_{1}|\xi|\left(\frac{\lambda_{2}}{\lambda_{3}}-1\right) e^{\lambda_{1} t}-i \lambda_{2}|\xi|\left(\frac{\lambda_{1}}{\lambda_{3}}-1\right) e^{\lambda_{2} t}+i|\xi|\left(\lambda_{1}-\lambda_{2}\right) e^{\lambda_{3} t}}{a_{3}\left(\lambda_{1}-\lambda_{2}\right)} \hat{c}_{1,0} \\
& -\frac{i \lambda_{1}|\xi|\left(\frac{\lambda_{2}}{\lambda_{4}}-1\right) e^{\lambda_{1} t}+\lambda_{1}|\xi|\left(\frac{\lambda_{1}}{\lambda_{4}}-1\right) e^{\lambda_{2} t}+i|\xi|\left(\lambda_{1}-\lambda_{2}\right) e^{\lambda_{4} t}}{a_{4}\left(\lambda_{1}-\lambda_{2}\right)} \hat{c}_{2,0}, \\
& \hat{c}_{1}=e^{\lambda_{3} t} \hat{c}_{1,0},
\end{aligned}
$$

and

$$
\hat{c}_{2}=e^{\lambda_{4} t} \hat{c}_{2,0}
$$

Now, by taking the curl for the second equation of (9.4), we have

$$
\partial_{t}(\nabla \times u)+\nu(\nabla \times u)=0 .
$$

Taking the Fourier transform for the above equation in $x$, we have

$$
\partial_{t}(\tilde{\xi} \times \hat{u})+\nu \tilde{\xi} \times \hat{u}=0
$$

Initial data is given as

$$
\left.(\tilde{\xi} \times \hat{u})\right|_{t=0}=\tilde{\xi} \times \hat{u}_{0}
$$


By solving the initial value problem (9.16) and (9.17), we have

$$
(\tilde{\xi} \times \hat{u})=e^{-\nu t} \tilde{\xi} \times \hat{u}_{0} .
$$

For $t \geq 0$ and $\xi \in \mathbb{R}^{3}$ with $|\xi| \neq 0$, one has the decomposition $\hat{u}=\tilde{\xi} \tilde{\xi} \cdot \hat{u}-\tilde{\xi} \times(\tilde{\xi} \times \hat{u})$. It is straightforward to get

$$
\begin{aligned}
\hat{u}=\frac{\lambda_{1} \lambda_{2} \xi}{i n_{\infty}|\xi|^{2}} & \left(\frac{e^{\lambda_{1} t}-e^{\lambda_{2} t}}{\lambda_{1}-\lambda_{2}}\right) \hat{\rho}_{0}+\left(\frac{\left(\lambda_{1} e^{\lambda_{1} t}-\lambda_{2} e^{\lambda_{2} t}\right.}{\lambda_{1}-\lambda_{2}}\right) \tilde{\xi} \tilde{\xi} \cdot \hat{u}_{0}-e^{-\nu t} \tilde{\xi} \times\left(\tilde{\xi} \times \hat{u}_{0}\right) \\
& +\frac{i \lambda_{1} \xi\left(\frac{\lambda_{2}}{\lambda_{3}}-1\right) e^{\lambda_{1} t}-i \lambda_{2} \xi\left(\frac{\lambda_{1}}{\lambda_{3}}-1\right) e^{\lambda_{2} t}+i \xi\left(\lambda_{1}-\lambda_{2}\right) e^{\lambda_{3} t}}{a_{3}\left(\lambda_{1}-\lambda_{2}\right)} \hat{c}_{1,0} \\
& -\frac{i \lambda_{1} \xi\left(\frac{\lambda_{2}}{\lambda_{4}}-1\right) e^{\lambda_{1} t}+i \lambda_{2} \xi\left(\frac{\lambda_{1}}{\lambda_{4}}-1\right) e^{\lambda_{2} t}+i \xi\left(\lambda_{1}-\lambda_{2}\right) e^{\lambda_{4} t}}{a_{4}\left(\lambda_{1}-\lambda_{2}\right)} \hat{c}_{2,0} .
\end{aligned}
$$

Then

$$
\begin{aligned}
\hat{u}= & \frac{\lambda_{1} \lambda_{2} \xi}{i n_{\infty}|\xi|^{2}}\left(\frac{e^{\lambda_{1} t}-e^{\lambda_{2} t}}{\lambda_{1}-\lambda_{2}}\right) \hat{\rho}_{0} \\
& +\left(\frac{\lambda_{1} e^{\lambda_{1} t}-\lambda_{2} e^{\lambda_{2} t}}{\lambda_{1}-\lambda_{2}}\right) \frac{\xi \otimes \xi}{|\xi|^{2}} \hat{u}_{0}+e^{-\nu t}\left(I_{3}-\frac{\xi \otimes \xi}{|\xi|^{2}}\right) \hat{u}_{0} \\
& +\frac{i \lambda_{1} \xi\left(\frac{\lambda_{2}}{\lambda_{3}}-1\right) e^{\lambda_{1} t}-\lambda_{2} \xi\left(\frac{\lambda_{1}}{\lambda_{3}}-1\right) e^{\lambda_{2} t}+i \xi\left(\lambda_{1}-\lambda_{2}\right) e^{\lambda_{3} t}}{a_{3}\left(\lambda_{1}-\lambda_{2}\right)} \hat{c}_{1,0} \\
& +\frac{-i \lambda_{1} \xi\left(\frac{\lambda_{2}}{\lambda_{4}}-1\right) e^{\lambda_{1} t}+i \lambda_{2} \xi\left(\frac{\lambda_{1}}{\lambda_{4}}-1\right) e^{\lambda_{2} t}-i \xi\left(\lambda_{1}-\lambda_{2}\right) e^{\lambda_{4} t}}{a_{4}\left(\lambda_{1}-\lambda_{2}\right)} \hat{c}_{2,0} .
\end{aligned}
$$

After summarizing the above computations on the explicit representation of Fourier transform of the solution $U=\left[\rho, u, c_{1}, c_{2}\right]$, we have

$$
\left[\begin{array}{c}
\hat{\rho}(t, \xi) \\
\hat{u}(t, \xi) \\
\hat{c}_{1}(t, \xi) \\
\hat{c}_{2}(t, \xi)
\end{array}\right]=\hat{G}(t, \xi)\left[\begin{array}{c}
\hat{\rho}(0, \xi) \\
\hat{u}(0, \xi) \\
\hat{c}_{1}(0, \xi) \\
\hat{c_{2}}(0, \xi)
\end{array}\right],
$$

where

$$
\hat{G}(t, \xi)=\left[\begin{array}{llll}
\hat{G}_{11} & \hat{G}_{12} & \hat{G}_{13} & \hat{G}_{14} \\
\hat{G}_{21} & \hat{G}_{22} & \hat{G}_{21} & \hat{G}_{21} \\
\hat{G}_{31} & \hat{G}_{32} & \hat{G}_{33} & \hat{G}_{34} \\
\hat{G}_{41} & \hat{G}_{42} & \hat{G}_{43} & \hat{G}_{44}
\end{array}\right]
$$


is the Green's matrix and it is the Fourier transform of the Green's function $G(t, \xi)=e^{t B}$. The elements of Green's matrix $\hat{G}(t, \xi)$ in $(9.19)$ are given by

$$
\begin{aligned}
& \hat{G}_{11}=\frac{\lambda_{1} e^{\lambda_{2} t}-\lambda_{2} e^{\lambda_{1} t}}{\lambda_{1}-\lambda_{2}}, \quad \hat{G}_{12}=-i n_{\infty} \frac{e^{\lambda_{1} t}-e^{\lambda_{2} t}}{\lambda_{1}-\lambda_{2}} \xi, \\
& \hat{G}_{13}=\frac{n_{\infty}|\xi|^{2}\left(\frac{\lambda_{2}}{\lambda_{3}}-1\right) e^{\lambda_{1} t}-n_{\infty}|\xi|^{2}\left(\frac{\lambda_{1}}{\lambda_{3}}-1\right) e^{\lambda_{2} t}+\frac{n_{\infty}|\xi|^{2}}{\lambda_{3}}\left(\lambda_{1}-\lambda_{2}\right) e^{\lambda_{3} t}}{a_{3}\left(\lambda_{1}-\lambda_{2}\right)}, \\
& \hat{G}_{14}=-\frac{i \lambda_{1} \xi\left(\frac{\lambda_{2}}{\lambda_{4}}-1\right) e^{\lambda_{1} t}+i \lambda_{2} \xi\left(\frac{\lambda_{1}}{\lambda_{4}}-1\right) e^{\lambda_{2} t}+i \xi\left(\lambda_{1}-\lambda_{2}\right) e^{\lambda_{4} t}}{a_{4}\left(\lambda_{1}-\lambda_{2}\right)}, \\
& \hat{G}_{21}=\frac{\lambda_{1} \lambda_{2} \xi}{i n_{\infty}|\xi|^{2}}\left(\frac{e^{\lambda_{1} t}-e^{\lambda_{2} t}}{\lambda_{1}-\lambda_{2}}\right) \\
& \hat{G}_{22}=\left(\frac{\lambda_{1} e^{\lambda_{1} t}-\lambda_{2} e^{\lambda_{2} t}}{\lambda_{1}-\lambda_{2}}\right) \frac{\xi \otimes \xi}{|\xi|^{2}}+e^{-\nu t}\left(I_{3}-\frac{\xi \otimes \xi}{|\xi|^{2}}\right), \\
& \hat{G}_{23}=\frac{i \lambda_{1} \xi\left(\frac{\lambda_{2}}{\lambda_{3}}-1\right) e^{\lambda_{1} t}-\lambda_{2} \xi\left(\frac{\lambda_{1}}{\lambda_{3}}-1\right) e^{\lambda_{2} t}+i \xi\left(\lambda_{1}-\lambda_{2}\right) e^{\lambda_{3} t}}{a_{3}\left(\lambda_{1}-\lambda_{2}\right)}, \\
& \hat{G}_{24}=\frac{-i \lambda_{1} \xi\left(\frac{\lambda_{2}}{\lambda_{4}}-1\right) e^{\lambda_{1} t}+i \lambda_{2} \xi\left(\frac{\lambda_{1}}{\lambda_{4}}-1\right) e^{\lambda_{2} t}-i \xi\left(\lambda_{1}-\lambda_{2}\right) e^{\lambda_{4} t}}{a_{4}\left(\lambda_{1}-\lambda_{2}\right)}, \\
& \hat{G}_{31}=G_{32}=G_{34}=0, \hat{G}_{33}=e^{\lambda_{3} t} \text {, } \\
& \hat{G}_{41}=G_{42}=G_{43}=0, \hat{G}_{44}=e^{\lambda_{4} t} \text {. }
\end{aligned}
$$

\subsubsection{Refined $L^{2}-L^{q}$ time-decay property.}

In this subsection, we use (9.19) to obtain some refined $L^{2}-L^{q}$ time-decay property for

$U=\left[\rho, u, c_{1}, c_{2}\right]$. To do so, we need to find the time-frequency pointwise estimate on $\hat{U}$ in the following Lemma:

Lemma 9.0.1. Suppose $U=\left[\rho, u, c_{1}, c_{2}\right]$ is the solution to the linear homogeneous system (9.4) with the initial data $\left.U\right|_{t=0}=U_{0}=\left(\rho_{0}, u_{0}, c_{1,0}, c_{2,0}\right)$. Then, there are constants $\epsilon>0$, $C>0, \lambda>0$ such that for all $t>0,|\xi| \leq \epsilon$,

$$
\begin{aligned}
&|\hat{\rho}(t, \xi)| \leq C\left(e^{-\lambda|\xi|^{2} t}+|\xi|^{2} e^{-\nu \lambda t}\right)\left|\hat{\rho}_{0}(\xi)\right|+C\left(|\xi| e^{-\lambda|\xi|^{2} t}+|\xi| e^{-\nu \lambda t}\right)\left|\hat{u}_{0}(\xi)\right| \\
&+ C|\xi|^{2}\left(e^{-\lambda|\xi|^{2} t}+e^{-\nu \lambda t}+e^{-\lambda t}\right)\left|\hat{c}_{1,0}(\xi), \hat{c}_{2,0}(\xi)\right| \\
&|\hat{u}(t, \xi)| \leq C|\xi|\left(e^{-\lambda|\xi|^{2} t}+e^{-\nu \lambda t}\right)\left|\hat{\rho}_{0}(\xi)\right|+C\left(|\xi|^{2} e^{-\lambda|\xi|^{2} t}+e^{-\nu \lambda t}\right)\left|\hat{u}_{0}(\xi)\right| \\
&+C\left(|\xi|^{3} e^{-\lambda|\xi|^{2} t}+C|\xi| e^{-\nu \lambda t}+C|\xi| e^{-\lambda t}\right)\left|\hat{c}_{1,0}(\xi), \hat{c}_{2,0}(\xi)\right|
\end{aligned}
$$




$$
\begin{gathered}
\left|\hat{c}_{1}\right| \leq C e^{-\lambda t}\left|\hat{c}_{1,0}(\xi)\right| \\
\left|\hat{c}_{2}\right| \leq C e^{-\lambda t}\left|\hat{c}_{2,0}(\xi)\right| .
\end{gathered}
$$

For all $t>0,|\xi| \geq \epsilon$,

$$
\begin{gathered}
|\hat{\rho}(t, \xi)| \leq C e^{-\lambda t}\left|\hat{U}_{0}\right| \\
|\hat{u}(t, \xi)| \leq C e^{-\lambda t}\left|\hat{U}_{0}\right| \\
\left|\hat{c}_{1}\right| \leq C e^{-\lambda t}\left|\hat{c}_{1,0}\right|
\end{gathered}
$$

and

$$
\left|\hat{c}_{2}\right| \leq C e^{-\lambda t}\left|\hat{c}_{2,0}\right|
$$

Proof. To obtain the upper bound of $\hat{U}(t, \xi)$, we have to estimate the elements of Green's matrix $\hat{G}(t, \xi)$ in (9.19). If $\nu^{2}-4 p^{\prime}\left(n_{\infty}\right)|\xi|^{2} \geq 0$, then $\lambda_{1,2}=-\frac{\nu}{2} \pm \frac{1}{2} \sqrt{\nu^{2}-4 p^{\prime}\left(n_{\infty}\right)|\xi|^{2}}$ are real. It is straightforward to obtain

$$
\begin{array}{r}
\lambda_{1} \sim-O(1)|\xi|^{2}, \\
\lambda_{2} \sim-\nu+O(1)|\xi|^{2}, \\
\lambda_{3,4} \sim-O(1),
\end{array}
$$

as $|\xi| \rightarrow 0$.

On other hand, if $\nu^{2}-4 p^{\prime}\left(n_{\infty}\right)|\xi|^{2} \leq 0$, then $\lambda_{1,2}=-\frac{\nu}{2} \pm \frac{\nu}{2} i \sqrt{\frac{4 p^{\prime}\left(n_{\infty}\right)}{\nu^{2}}|\xi|^{2}-1}$ are complex conjugates. Moreover, we have

$$
\begin{array}{r}
\left|\lambda_{1,2}\right| \sim O(1)|\xi|, \\
\lambda_{1}-\lambda_{2} \sim i O(1)|\xi|, \\
\lambda_{3,4} \sim-O(1)|\xi|^{2},
\end{array}
$$

as $|\xi| \rightarrow \infty$. Then, there exists $\epsilon \leq \sqrt{\frac{\nu^{2}}{4 p^{\prime}\left(n_{\infty}\right)}} \leq R$, with $0<\epsilon \ll 1 \ll R<\infty$ such that we 
can estimate Green's matrix $\hat{G}(t, \xi)$ as follows:

$$
\begin{aligned}
\left|\hat{G}_{11}\right| & \leq C\left(e^{-\lambda|\xi|^{2} t}+|\xi|^{2} e^{-\nu \lambda t}\right) \\
\left|\hat{G}_{12}\right| & \leq C|\xi|\left(e^{-\lambda|\xi|^{2} t}+e^{-\nu \lambda t}\right) \\
\left|\hat{G}_{13}\right| & \leq C|\xi|^{2}\left(e^{-\lambda|\xi|^{2} t}+e^{-\nu \lambda t}+e^{-\lambda t}\right) \\
\left|\hat{G}_{14}\right| & \leq C|\xi|^{2}\left(e^{-\lambda|\xi|^{2} t}+e^{-\nu \lambda t}+e^{-\lambda t}\right) \\
\left|\hat{G}_{21}\right| & \leq C|\xi|\left(e^{-\lambda|\xi|^{2} t}+e^{-\nu \lambda t}\right) \\
\left|\hat{G}_{22}\right| & \leq C e^{-\nu t}+C\left(|\xi|^{2} e^{-\lambda|\xi|^{2} t}+e^{-\nu \lambda t}\right) \\
& \leq C|\xi|^{2} e^{-\lambda|\xi|^{2} t}+C e^{-\nu \lambda t} \\
\left|\hat{G}_{23}, G_{24}\right| & \leq C\left(|\xi|^{3} e^{-\lambda|\xi|^{2} t}+C|\xi| e^{-\nu \lambda t}+C|\xi| e^{-\lambda t}\right) \\
\left|\hat{G}_{33}\right| & \leq C e^{-\lambda t} \\
\left|\hat{G}_{44}\right| & \leq C e^{-\lambda t}
\end{aligned}
$$

as $|\xi| \leq \epsilon$, and

$$
\left|\hat{G}_{i j}\right| \leq C e^{-\lambda t}, \quad 1 \leq i, j \leq 4
$$

as $|\xi|>R$.

When the eigenvalues $\lambda_{1}$ and $\lambda_{2}$ coalesce, since the real part is negative, we have $t e^{-\frac{\nu}{2} t}$ in the solution, but this decays exponentially. Then, we get $t e^{-\frac{\nu}{2} t} \leq e^{-\lambda t}$. Now, we can estimate $\hat{U}=\left[\hat{\rho}, \hat{u}, \hat{c}_{2}, \hat{c}_{2}\right]$ as follows

$$
\begin{aligned}
|\hat{\rho}(t, \xi)| & =\left|\hat{G}_{11} \hat{\rho}_{0}+\hat{G}_{12} \hat{u}_{0}+\hat{G}_{13} \hat{c}_{1,0}+\hat{G}_{14} \hat{c}_{2,0}\right| \\
& \leq\left|\hat{G}_{11}\right|\left|\hat{\rho}_{0}\right|+\left|\hat{G}_{12}\right|\left|\hat{u}_{0}\right|+\left|\hat{G}_{13}\right|\left|\hat{c}_{1,0}\right|+\left|\hat{G}_{14}\right|\left|\hat{c}_{2,0}\right| \\
& \leq C\left(e^{-\lambda|\xi|^{2} t}+|\xi|^{2} e^{-\nu \lambda t}\right)\left|\hat{\rho}_{0}(\xi)\right|+C\left(|\xi| e^{-\lambda|\xi|^{2} t}+|\xi| e^{-\nu \lambda t}\right)\left|\hat{u}_{0}(\xi)\right| \\
& +C|\xi|^{2}\left(e^{-\lambda|\xi|^{2} t}+e^{-\nu \lambda t}+e^{-\lambda t}\right)\left|\hat{c}_{1,0}, \hat{c}_{2,0}\right|, \\
|\hat{u}(t, \xi)| & =\left|\hat{G}_{21} \hat{\rho}_{0}+\hat{G}_{22} \hat{u}_{0}\right|+\hat{G}_{23} \hat{c}_{1,0}+\hat{G}_{24} \hat{c}_{2,0} \mid \\
& \leq\left|\hat{G}_{21}\right|\left|\hat{\rho}_{0}\right|+\left|\hat{G}_{22}\right|\left|\hat{u}_{0}\right|+\left|\hat{G}_{23}\right|\left|\hat{c}_{1,0}\right|+\left|\hat{G}_{24}\right|\left|\hat{c}_{2,0}\right| \\
& \leq C|\xi|\left(e^{-\lambda|\xi|^{2} t}+e^{-\nu \lambda t}\right)\left|\hat{\rho}_{0}(\xi)\right|+C\left(|\xi|^{2} e^{-\lambda|\xi|^{2} t}+e^{-\nu \lambda t}\right)\left|\hat{u}_{0}(\xi)\right| \\
& +C\left(|\xi|^{3} e^{-\lambda|\xi|^{2} t}+C|\xi| e^{-\nu \lambda t}+C|\xi| e^{-\lambda t}\right)\left|\hat{c}_{1,0}, \hat{c}_{2,0}\right|,
\end{aligned}
$$




$$
\begin{aligned}
& \left|\hat{c}_{1}(t, \xi)\right| \leq C e^{-\lambda t}\left|\hat{c}_{1,0}\right|, \\
& \left|\hat{c}_{2}(t, \xi)\right| \leq C e^{-\lambda t}\left|\hat{c}_{2,0}\right|,
\end{aligned}
$$

for $|\xi| \leq \epsilon$. Finally, (9.24)-(9.27) can be proved in the completely same way as for (9.20)(9.23). This completes the proof of Lemma (9.0.1).

Theorem 9.0.2. Let $2 \leq q \leq \infty$, and let $m \geq 0$ be an integer. Assume $U=e^{B t} U_{0}$ is the solution to the Cauchy problem (9.4)-(9.5). Then for any $t \geq 0, U=\left[\rho, u, c_{1}, c_{2}\right]$ satisfies:

$$
\begin{gathered}
\left\|\nabla^{m} \rho(t)\right\|_{L^{q}} \leq C(1+t)^{-\frac{3}{2}\left(1-\frac{1}{q}\right)-\frac{m}{2}}\left\|U_{0}\right\|_{L^{1}}+e^{-\lambda t}\left\|\nabla^{m+\left[3\left(\frac{1}{2}-\frac{1}{q}\right)\right]_{+}} U_{0}\right\|, \\
\left\|\nabla^{m} u(t)\right\|_{L^{q}} \leq C(1+t)^{-\frac{3}{2}\left(1-\frac{1}{q}\right)-\frac{m+1}{2}}\left\|U_{0}\right\|_{L^{1}}+e^{-\lambda t}\left\|\nabla^{m+\left[3\left(\frac{1}{2}-\frac{1}{q}\right)\right]_{+}} U_{0}\right\|, \\
\left\|\nabla^{m} c_{1}(t)\right\|_{L^{q}} \leq C e^{-\lambda t}\left(\left\|c_{1,0}\right\|+\left\|\nabla^{m+\left[3\left(\frac{1}{2}-\frac{1}{q}\right)\right]_{+}} c_{1,0}\right\|\right), \\
\left\|\nabla^{m} c_{2}(t)\right\|_{L^{q}} \leq C e^{-\lambda t}\left(\left\|c_{2,0}\right\|+\left\|\nabla^{m+\left[3\left(\frac{1}{2}-\frac{1}{q}\right)\right]_{+}} c_{2,0}\right\|\right),
\end{gathered}
$$

where $C=C(m, q)$ and $\left[3\left(\frac{1}{2}-\frac{1}{q}\right)\right]_{+}$is defined by

$$
\left[3\left(\frac{1}{2}-\frac{1}{q}\right)\right]_{+}=\left\{\begin{array}{l}
0 \quad \text { if } q=2 \\
{\left[3\left(\frac{1}{2}-\frac{1}{q}\right)\right]_{-}+1 \quad \text { otherwise, }}
\end{array}\right.
$$

where $[.]_{-}$denotes the integer part of the argument.

Proof. Take $2 \leq q \leq \infty$ and let $m \geq 0$ be an integer. Set $U=e^{B t} U_{0}$. Using the HausdorffYoung inequality and (9.20), we prove (9.28) as follows,

$$
\left\|\nabla^{m} \rho(t)\right\|_{L^{q}\left(\mathbb{R}_{x}^{3}\right)} \leq C\left\||\xi|^{m} \hat{\rho}(\xi, t)\right\|_{L^{q^{\prime}\left(\mathbb{R}_{\xi}^{3}\right)}} \leq C\left\||\xi|^{m} \hat{\rho}(\xi, t)\right\|_{L^{q^{\prime}}(|\xi| \leq \epsilon)}+C\left\||\xi|^{m} \hat{\rho}(\xi, t)\right\|_{L^{q^{\prime}(|\xi| \geq \epsilon)}},
$$

where $\frac{1}{q}+\frac{1}{q^{\prime}}=1$. 
We estimate the first term of (9.32) as

$$
\begin{aligned}
& \left\||\xi|^{m} \hat{\rho}(t, \xi)\right\|_{L^{q \prime}(|\xi| \leq \epsilon)}^{q^{\prime}} \leq C \int_{|\xi| \leq \epsilon}\left(|\xi|^{m q^{\prime}} e^{-q^{\prime} \lambda|\xi|^{2} t}+|\xi|^{(m+2) q^{\prime}} e^{-q^{\prime} \nu \lambda t}\right)\left|\hat{\rho}_{0}(\xi)\right|^{q^{\prime}} d \xi \\
& +C \int_{|\xi| \leq \epsilon}\left(|\xi|^{m q^{\prime}+q^{\prime}} e^{-q^{\prime} \lambda|\xi|^{2} t}+|\xi|^{m q^{\prime}+q^{\prime}} e^{-q^{\prime} \nu \lambda t}\right)\left|\hat{u}_{0}(\xi)\right|^{q^{\prime}} d \xi \\
& +C \int_{\xi \leq \epsilon}\left(|\xi|^{(m+2) q^{\prime}} e^{-q^{\prime} \lambda|\xi|^{2}(t+1)}+|\xi|^{(m+2) q^{\prime}} e^{-q^{\prime} \nu \lambda t}+|\xi|^{(m+2) q^{\prime}} e^{-q^{\prime} \lambda t}\left|\hat{c}_{1,0}(\xi), \hat{c}_{2,0}(\xi)\right|^{q^{\prime}}\right) d \xi \\
& \leq C \sup _{\xi}\left|\hat{\rho}_{0}\right|^{q \prime} \int_{|\xi| \leq \epsilon}\left(|\xi|^{m q^{\prime}} e^{-q^{\prime} \lambda|\xi|^{2}(1+t)+q^{\prime} \lambda|\xi|^{2}}+|\xi|^{m q^{\prime}+2 q^{\prime}} e^{-q^{\prime} \nu \lambda t}\right) d \xi \\
& +C \sup _{\xi}\left|\hat{u}_{0}\right|^{q \prime} \int_{|\xi| \leq \epsilon}\left(|\xi|^{m q^{\prime}+q^{\prime}} e^{-q^{\prime} \lambda|\xi|^{2}(1+t)+q^{\prime} \lambda|\xi|^{2}}+|\xi|^{m q^{\prime}+q^{\prime}} e^{-q^{\prime} \nu \lambda t}\right) d \xi \\
& +C \sup _{\xi}\left|\hat{c}_{1,0}(\xi), \hat{c}_{2,0}\right|^{q \prime} \int_{|\xi| \leq \epsilon}\left(|\xi|^{(m+2) q^{\prime}} e^{-q^{\prime} \lambda|\xi|^{2}(t+1)}+|\xi|^{(m+2) q^{\prime}} e^{-q^{\prime} \nu \lambda t}+|\xi|^{(m+2) q^{\prime}} e^{-q^{\prime} \lambda t}\right) d \xi \\
& \leq C(1+t)^{-\frac{m q^{\prime}+3}{2}}\left\|\rho_{0}\right\|_{L^{1}}^{q^{\prime}}+C(1+t)^{-\frac{m q^{\prime}+q^{\prime}+3}{2}}\left\|u_{0}\right\|_{L^{1}}^{q^{\prime}}+C e^{-q^{\prime} \nu \lambda t}\left\|U_{0}\right\|_{L^{1}}^{q^{\prime}} \\
& +C(1+t)^{-\frac{m q^{\prime}+2 q^{\prime}+3}{2}}\left\|\left[c_{1,0}, c_{2,0}\right]\right\|\left\|_{L^{1}}^{q^{\prime}}+C e^{-q^{\prime} \lambda t}\right\|\left[c_{1,0}, c_{2,0}\right] \|_{L^{1}}^{q^{\prime}}
\end{aligned}
$$

Thus,

$$
\begin{aligned}
& \left\||\xi|^{m} \hat{\rho}(t, \xi)\right\|_{L^{q^{\prime}}(|\xi| \leq \epsilon)} \leq C(1+t)^{-\frac{3}{2 q^{\prime}}-\frac{m}{2}}\left\|\rho_{0}\right\|_{L^{1}}+C(1+t)^{-\frac{3}{2 q^{\prime}}-\left(\frac{m+1}{2}\right)}\left\|u_{0}\right\|_{L^{1}} \\
& +C(1+t)^{-\frac{3}{2 q^{\prime}}-\left(\frac{m+2}{2}\right)}\left\|\left[c_{1,0}, c_{2,0}\right]\right\|_{L^{1}}+C e^{-\nu \lambda t}\left\|U_{0}\right\|_{L^{1}}+C e^{-\lambda t}\left\|\left[c_{1,0}, c_{2,0}\right]\right\|_{L^{1}} \\
& \leq C(1+t)^{-\frac{3}{2}\left[1-\frac{1}{q}\right]-\frac{m}{2}}\left\|U_{0}\right\|_{L^{1}} .
\end{aligned}
$$

Now, we estimate the second term of (9.32) using the Hölder inequality $\frac{1}{q^{\prime}}=\frac{1}{2}+\frac{2-q^{\prime}}{2 q^{\prime}}$ and fixing $\epsilon>0$ small enough, we obtain

$$
\begin{gathered}
\left\||\xi|^{m} \hat{\rho}(\xi, t)\right\|_{L^{q^{\prime}}(|\xi| \geq \epsilon)} \leq C \int_{|\xi| \geq \epsilon}|\xi|^{m q^{\prime}} e^{-q^{\prime} \lambda t}\left|\hat{U}_{0}(\xi)\right|^{q^{\prime}} d \xi \\
\leq C e^{-\lambda t}\left\||\xi|^{-(3+\epsilon)}\right\|^{\frac{2-q^{\prime}}{2 q^{\prime}}}\left\||\xi|^{(3+\epsilon) \frac{2-q^{\prime}}{2 q^{\prime}}+m} \hat{U}_{0}(\xi)\right\| \\
\leq C e^{-\lambda t}\left\|\nabla^{m+3\left[\frac{1}{2}-\frac{1}{q}\right]-} U_{0}\right\|,
\end{gathered}
$$

after plugging (9.33) and (9.34) into (9.32) implies (9.28).

To prove (9.29), it similarly holds that

$$
\left\|\nabla^{m} u(t)\right\|_{L^{q}\left(\mathbb{R}_{x}^{3}\right)} \leq C\left\||\xi|^{m} \hat{u}(\xi, t)\right\|_{L^{q^{\prime}\left(\mathbb{R}_{\xi}^{3}\right)}} \leq C\left\||\xi|^{m} \hat{u}(\xi, t)\right\|_{L^{q^{\prime}(|\xi| \leq \epsilon)}}+C\left\||\xi|^{m} \hat{u}(\xi, t)\right\|_{L^{q^{\prime}(|\xi| \geq \epsilon)}} .
$$


By using (9.21) we estimate the first term of (9.35) as

$$
\begin{aligned}
& \left\||\xi|^{m} \hat{u}(t, \xi)\right\|_{L^{q^{\prime}(|\xi| \leq \epsilon)}}^{q^{\prime}} \leq C \int_{|\xi| \leq \epsilon}\left(|\xi|^{m q^{\prime}+q^{\prime}}\left(e^{-q^{\prime} \lambda|\xi|^{2}(t+1)}+e^{-q^{\prime} \nu \lambda t}\right)\left|\hat{\rho}_{0}(\xi)\right|^{q \prime} d \xi\right. \\
& +C \int_{\xi \leq \epsilon}\left(|\xi|^{(m+2) q^{\prime}} e^{-q^{\prime} \lambda|\xi|^{2}(t+1)}+e^{-q^{\prime} \nu \lambda t}\right)\left|\hat{u}_{0}(\xi)\right|^{q \prime} d \xi \\
& +C \int_{\xi \leq \epsilon}\left(|\xi|^{(m+3) q^{\prime}} e^{-q^{\prime} \lambda|\xi|^{2}(t+1)}+|\xi|^{m q^{\prime}+q^{\prime}} e^{-q^{\prime} \nu \lambda t}+|\xi|^{m q^{\prime}+q^{\prime}} e^{-q^{\prime} \lambda t}\right)\left|\hat{c}_{1,0}(\xi), \hat{c}_{2,0}(\xi)\right|^{q^{\prime}} d \xi \\
& \leq C(1+t)^{-\frac{m q^{\prime}+q^{\prime}+3}{2}}\left\|\rho_{0}\right\|_{L^{1}}^{q^{\prime}}+(1+t)^{-\frac{m q^{\prime}+2 q^{\prime}+3}{2}}\left\|u_{0}\right\|_{L^{1}}^{q^{\prime}}+C(1+t)^{-\frac{m q^{\prime}+3 q^{\prime}+3}{2}}\left\|\left[c_{1,0}, c_{2,0}\right]\right\|_{L^{1}}^{q^{\prime}} \\
& +C e^{-q^{\prime} \nu \lambda t}\left\|U_{0}\right\|_{L^{1}}^{q^{\prime}}+C e^{-q^{\prime} \lambda t}\left\|\left[c_{1,0}, c_{2,0}\right]\right\|_{L^{1}}^{q^{\prime}} .
\end{aligned}
$$

It follows that

$$
\begin{aligned}
& \left\||\xi|^{m} \hat{u}(t, \xi)\right\|_{L^{q^{\prime}}(|\xi| \leq \epsilon)} \leq C(1+t)^{-\frac{3}{2 q^{\prime}}-\frac{m+1}{2}} \|\left[\rho_{0}\left\|_{L^{1}}+(1+t)^{-\frac{3}{2 q^{\prime}}-\frac{m+2}{2}}\right\| u_{0} \|_{L^{1}}\right. \\
& +(1+t)^{-\frac{3}{2 q^{\prime}}-\frac{m+3}{2}}\left\|\left[c_{1,0}, c_{2,0}\right]\right\|_{L^{1}}+C e^{-\nu \lambda t}\left\|U_{0}\right\|_{L^{1}}+C e^{-\lambda t}\left\|\left[c_{1,0}, c_{2,0}\right]\right\|_{L^{1}} \\
& \leq C(1+t)^{-\frac{3}{2}\left[1-\frac{1}{q}\right]-\frac{m+1}{2}}\left\|\rho_{0}\right\|_{L^{1}}+(1+t)^{-\frac{3}{2}\left[1-\frac{1}{q}\right]-\frac{m+2}{2}}\left\|u_{0}\right\|_{L^{1}}+C e^{-\nu \lambda t}\left\|U_{0}\right\|_{L^{1}} \\
& +(1+t)^{-\frac{3}{2}\left[1-\frac{1}{q}\right]-\frac{m+3}{2}}\left\|\left[c_{1,0}, c_{2,0}\right]\right\|_{L^{1}}+C e^{-\lambda t}\left\|\left[c_{1,0}, c_{2,0}\right]\right\|_{L^{1}} \\
& \leq C(1+t)^{-\frac{3}{2}\left[1-\frac{1}{q}\right]-\frac{m+1}{2}}\left\|\left[\rho_{0}, u_{0}, c_{1,0}, c_{2,0}\right]\right\|_{L^{1}} .
\end{aligned}
$$

Similarly to obtaining (9.34), one has

$$
\left\||\xi|^{m} \hat{u}(\xi, t)\right\|_{L^{q^{\prime}}(|\xi| \geq \epsilon)} \leq C e^{-\lambda t}\left\|\nabla^{m+3\left[\frac{1}{2}-\frac{1}{q}\right]}-U_{0}\right\|
$$

Thus, plugging (9.37) and (9.36) into (9.35) implies (9.29). We prove (9.30) and (9.31) in the similar way. This completes the proof of Theorem 9.0.2.

Corollary 9.0.3. Assume that $U=e^{B t} U_{0}$ is the solution to the Cauchy problem (9.4) with initial data $U_{0}=\left[\rho_{0}, u_{0}, c_{1,0}, c_{2,0}\right]$. Then $U=\left[\rho, u, c_{1}, c_{2}\right]$ satisfies the following:

$$
\begin{gathered}
\|\rho(t)\| \leq C(1+t)^{-\frac{3}{4}}\left\|U_{0}\right\|_{L^{1}}+e^{-\lambda t}\left\|U_{0}\right\|, \\
\|u(t)\| \leq C(1+t)^{-\frac{5}{4}}\left\|U_{0}\right\|_{L^{1}}+e^{-\lambda t}\left\|U_{0}\right\|, \\
\|\rho(t)\|_{L^{\infty}} \leq C(1+t)^{-\frac{3}{2}}\left\|U_{0}\right\|_{L^{1}}+e^{-\lambda t}\left\|\nabla^{2} U_{0}\right\|, \\
\|u(t)\|_{L^{\infty}} \leq C(1+t)^{-2}\left\|U_{0}\right\|_{L^{1}}+e^{-\lambda t}\left\|\nabla^{2} U_{0}\right\|, \\
\left\|c_{1}(t)\right\| \leq C e^{-\lambda t}\left\|c_{1,0}\right\|,
\end{gathered}
$$




$$
\begin{gathered}
\left\|c_{2}(t)\right\| \leq C e^{-\lambda t}\left\|c_{2,0}\right\|, \\
\left\|c_{1}(t)\right\|_{L^{\infty}} \leq C e^{-\lambda t}\left(\left\|c_{1,0}\right\|_{L^{2}}+\left\|\nabla^{2} c_{1,0}\right\|_{L^{2}}\right), \\
\left\|c_{2}(t)\right\|_{L^{\infty}} \leq C e^{-\lambda t}\left(\left\|c_{2,0}\right\|_{L^{2}}+\left\|\nabla^{2} c_{2,0}\right\|_{L^{2}}\right) .
\end{gathered}
$$




\section{Chapter 10}

\section{Time-Decay Rates for the nonlinear}

\section{system}

In this chapter, we will prove Proposition 7.0.2 and Proposition 7.0.3. The main idea is to combine the energy estimates and spectral analysis. We apply the linear $L^{2}-L^{q}$ time-decay property of the homogeneous system (9.4) studied in the previous chapter to the nonlinear case. We need the mild form of the original nonlinear Cauchy problem (7.2). Throughout this chapter, we suppose that $U=\left[\rho, u, c_{1}, c_{2}\right]$ is the solution to the Cauchy problem (7.2) with initial data $U_{0}=\left(\rho_{0}, u_{0}, c_{1,0}, c_{2,0}\right)$ satisfying (7.3).

Then, by Duhamel's principle, the solution $U=\left[\rho, u, c_{1}, c_{2}\right]$ can be formally written as

$$
U(t)=e^{B t} U_{0}+\int_{0}^{t} e^{(t-s) B}\left[g_{1}, g_{2}, g_{3}, g_{4}\right] d s,
$$

where $e^{B t}, t \geq 0$, is called the linear solution operator and the nonlinear source term takes the form (9.3).

\subsubsection{Decay rates for the energy functional and high-order en- ergy functional}

In this subsection, we will prove the decay rate for the energy functional $\|U(t)\|_{N}^{2}$ and the decay rate for the high-order energy functional $\|\nabla U(t)\|_{N}^{2}$. For that, we investigate the timedecay rates of solutions in Proposition 7.0.1 under an extra condition (7.8). 
Proof of Proposition 7.0.2. Suppose $\epsilon_{N+1}\left(U_{0}\right)$ is sufficiently small. Then, from Proposition 7.0.1 the solution $U=\left[\rho, u, c_{1}, c_{2}\right]$ satisfies:

$$
\frac{d}{d t} \mathcal{E}_{N}(U(t))+\lambda_{1} \mathcal{D}_{N}(U(t))+\lambda_{2} \mathcal{D}_{N}^{h}(U(t)) \leq 0
$$

for any $t \geq 0$, where $\mathcal{E}_{N} U(t) \sim\left\|\left[\rho, u, c_{1}, c_{2}\right]\right\|_{N}^{2}$ denotes the energy functional and $\mathcal{D}_{N} U(t) \sim$ $\left\|\left[\nabla\left(c_{1}, c_{2}\right)\right]\right\|_{N}^{2}$ and $\mathcal{D}_{N}^{h} U(t) \sim\|\nabla \rho\|_{N-1}^{2}+\left\|\left[u, c_{1}, c_{2}\right]\right\|_{N}^{2}$ the dissipation rates.

Now, we begin with the time-weighted estimate and iteration for inequality (10.2). Let $l \geq 0$. Multiplying $(10.2)$ by $(1+t)^{l}$ and integrating over $[0, t]$ give

$$
\begin{aligned}
(1+t)^{l} \mathcal{E}_{N} U(t)+ & \lambda_{1} \int_{0}^{t}(1+s)^{l} \mathcal{D}_{N}(U(s)) d s+\lambda_{2} \int_{0}^{t}(1+s)^{l} \mathcal{D}_{N}^{h}(U(s)) d s \\
& \leq \mathcal{E}_{N}\left(U_{0}\right)+l \int_{0}^{t}(1+s)^{l-1} \mathcal{E}_{N} U(s) d s \\
& \leq \mathcal{E}_{N}\left(U_{0}\right)+C l \int_{0}^{t}(1+s)^{l-1}\left(\mathcal{D}_{N-1} U(s)+\mathcal{D}_{N}^{h}(U(s))+\|\rho(s)\|^{2}\right) d s
\end{aligned}
$$

where we have used

$$
\mathcal{E}_{N} U(t) \leq C \mathcal{D}_{N-1} U(t)+C \mathcal{D}_{N}^{h}(U(t))+\|\rho(t)\|^{2} .
$$

Using (10.2) again, we have

$$
\mathcal{E}_{N+1}(U(t))+\lambda_{1} \int_{0}^{t} \mathcal{D}_{N+1}(U(t))+\lambda_{2} \int_{0}^{t} \mathcal{D}_{N+1}^{h}(U(t)) \leq \mathcal{E}_{N+1}\left(U_{0}\right),
$$

and

$$
\begin{aligned}
& (1+t)^{l-1} \mathcal{E}_{N+1} U(t)+\lambda_{1} \int_{0}^{t}(1+s)^{l-1} \mathcal{D}_{N+1}(U(s)) d s+\lambda_{2} \int_{0}^{t}(1+s)^{l-1} \mathcal{D}_{N+1}^{h}(U(s)) d s \\
& \leq \mathcal{E}_{N+1}\left(U_{0}\right)+C(l-1) \int_{0}^{t}(1+s)^{l-2} \mathcal{E}_{N+1} U(s) d s \\
& \leq \mathcal{E}_{N+1}\left(U_{0}(t)\right)+C(l-1) \int_{0}^{t}(1+s)^{l-2}\left(\mathcal{D}_{N} U(s)+C \mathcal{D}_{N+1}^{h}(U(s))+\|\rho(s)\|^{2}\right) d s .
\end{aligned}
$$

Then, for $1<l<2$, it follows by iterating the previous estimates that

$$
\begin{gathered}
(1+t)^{l} \mathcal{E}_{N} U(t)+\lambda_{1} \int_{0}^{t}(1+s)^{l} \mathcal{D}_{N}(U(s)) d s+\lambda_{2} \int_{0}^{t}(1+s)^{l} \mathcal{D}_{N}^{h}(U(s)) d s \\
\leq \mathcal{E}_{N+1}\left(U_{0}\right)+C \int_{0}^{t}(1+s)^{l-1}\|\rho(s)\|^{2} d s
\end{gathered}
$$


On the other hand, to estimate the integral term on the right-hand side of the previous inequality, let us define

$$
\mathcal{E}_{N, \infty}(U(t))=\sup _{0 \leq s \leq T}(1+t)^{\frac{3}{2}} \mathcal{E}_{N} U(t)
$$

By applying the linear estimate on $\rho$ in (9.38) to the mild form (10.1), one has

$$
\begin{aligned}
\|\rho(t)\| \leq & C(1+t)^{\frac{-3}{4}}\left\|U_{0}\right\|_{L^{1}}+C e^{-\lambda t}\left\|U_{0}\right\|+C \int_{0}^{t}(1+t-s)^{\frac{-3}{4}}\left\|\left[g_{1}, g_{2}, g_{3}, g_{4}\right](s)\right\|_{L^{1}} d s \\
& +C \int_{0}^{t} e^{-\lambda(t-s)}\left\|\left[g_{1}, g_{2}, g_{3}, g_{4}\right](s)\right\| d s
\end{aligned}
$$

Recall the definitions (9.3) of $g_{1}$ and $g_{2}$. It is direct to check that for any $0 \leq s \leq t$,

$$
\left\|g_{1}, g_{2}, g_{3}, g_{4}(s)\right\|_{L^{1} \cap L^{2}} \leq C \mathcal{E}_{N} U(t) \leq C(1+s)^{\frac{-3}{2}} \mathcal{E}_{N, \infty} U(t)
$$

Putting this into (10.4) gives

$$
\|\rho(t)\| \leq C(1+t)^{\frac{-3}{4}}\left(\left\|U_{0}\right\|_{L^{1} \cap L^{2}}+\mathcal{E}_{N, \infty} U(t)\right)
$$

Next, we prove the uniform-in-time bound of $\left.\mathcal{E}_{N, \infty} U(t)\right)$ which implies the decay rates of the energy functional $\mathcal{E}_{N} U(t)$. In fact, by taking $l=\frac{3}{2}+\epsilon$ in (10.3) where $\epsilon>0$ is small enough, it follows that

$$
\begin{gathered}
(1+t)^{\frac{3}{2}+\epsilon} \mathcal{E}_{N} U(t)+\lambda_{1} \int_{0}^{t}(1+s)^{\frac{3}{2}+\epsilon} \mathcal{D}_{N}(U(s)) d s+\lambda_{2} \int_{0}^{t}(1+s)^{\frac{3}{2}+\epsilon} \mathcal{D}_{N}^{h}(U(s)) d s \\
\leq \mathcal{E}_{N+1}\left(U_{0}\right)+C \int_{0}^{t}(1+s)^{\frac{1}{2}+\epsilon}\|\rho(s)\|^{2} d s
\end{gathered}
$$

Here, using (10.5), we obtain

$$
\left.\int_{0}^{t}(1+s)^{\frac{1}{2}+\epsilon}\|\rho(t)\|^{2} d s \leq C(1+t)^{\epsilon}\left(\mathcal{E}_{N, \infty}^{2} U(t)\right)+\left\|U_{0}\right\|_{L^{1} \cap L^{2}}^{2}\right) .
$$

Therefore, it follows that

$$
\begin{gathered}
(1+t)^{\frac{3}{2}+\epsilon} \mathcal{E}_{N} U(t)+\lambda_{1} \int_{0}^{t}(1+s)^{\frac{3}{2}+\epsilon} \mathcal{D}_{N}(U(s)) d s+\lambda_{2} \int_{0}^{t}(1+s)^{\frac{3}{2}+\epsilon} \mathcal{D}_{N}^{h}(U(s)) d s \\
\left.\leq \mathcal{E}_{N+1}\left(U_{0}\right)+C(1+t)^{\epsilon}\left(\mathcal{E}_{N, \infty}^{2} U(t)\right)+\left\|U_{0}\right\|_{L^{1} \cap L^{2}}^{2}\right)
\end{gathered}
$$

which implies

$$
(1+t)^{\frac{3}{2}} \mathcal{E}_{N} U(t) \leq C\left(\mathcal{E}_{N+1}\left(U_{0}\right)+\left\|\rho_{0}, u_{0}\right\|_{L^{1}}^{2}+\mathcal{E}_{N, \infty}^{2} U(t)\right)
$$


and thus

$$
\mathcal{E}_{N, \infty} U(t) \leq C\left(\epsilon_{N+1}^{2}\left(U_{0}\right)+\mathcal{E}_{N, \infty}^{2} U(t)\right) .
$$

Since $\epsilon_{N+1}^{2}\left(U_{0}\right)>0$ is sufficiently small, it holds that $\left.\mathcal{E}_{N, \infty} U(t)\right) \leq C \epsilon_{N+1}^{2}\left(U_{0}\right)$ for any $t \geq 0$, which gives $\|U(s)\|_{N} \leq C\left(\mathcal{E}_{N} U(t)\right)^{\frac{1}{2}} \leq C \epsilon_{N+1}\left(U_{0}\right)(1+t)^{-\frac{3}{4}}$. This proves (7.9).

Now, we estimate the high-order energy functional. By comparing the definitions of $\mathcal{E}_{N} U(t)$, $\mathcal{D}_{N} U(t)$, and $\mathcal{D}_{N}^{h} U(t)$, it follows from (10.2) that

$$
\frac{d}{d t}\|\nabla U(t)\|_{N}^{2}+\lambda\|\nabla U(t)\|_{N}^{2} \leq C\|\nabla \rho(t)\|^{2},
$$

which implies

$$
\|\nabla U(t)\|_{N}^{2} \leq e^{-\lambda t}\left\|\nabla U_{0}\right\|_{N}^{2}+C \int_{0}^{t} e^{-\lambda(t-s)}\|\nabla \rho(s)\|^{2} d s,
$$

for any $t \geq 0$. To estimate the time integral term on the (r.h.s.) of the above inequality, one can apply the linear estimate (9.28) to the mild form (10.1) of the solution $U(t)$ so that

$$
\begin{aligned}
\|\nabla \rho(t)\| \leq & C(1+t)^{\frac{-5}{4}}\left\|U_{0}\right\|_{L^{1}}+C e^{-\lambda t}\left\|\nabla U_{0}\right\|+C \int_{0}^{t}(1+t-s)^{\frac{-5}{4}}\left\|\left[g_{1}, g_{2}, g_{3}, g_{4}\right](s)\right\|_{L^{1}} d s \\
& +C \int_{0}^{t} e^{-\lambda(t-s)}\left\|\nabla\left[g_{1}, g_{2}, g_{3}, g_{4}\right](s)\right\| d s .
\end{aligned}
$$

Recall the definition (9.3) of $g_{1}, g_{2}, g_{3}$ and $g_{4}$. It is straightforward to check that for any $0 \leq s \leq t$

$$
\left\|\left[g_{1}, g_{2}, g_{3}, g_{4}(s)\right]\right\|_{L^{1} \cap \dot{H}^{1}} \leq C \mathcal{E}_{N} U(s) \leq C \epsilon_{N+1}^{2}\left(U_{0}\right)(1+s)^{\frac{-3}{2}} .
$$

Putting this into (10.7) gives

$$
\|\nabla \rho(t)\| \leq C \epsilon_{N+1}\left(U_{0}\right)(1+t)^{\frac{-5}{4}} .
$$

Then, by using (10.8) in (10.6), we have

$$
\|\nabla U(t)\|_{N}^{2} \leq e^{-\lambda t}\left\|\nabla U_{0}\right\|_{N}^{2}+C \epsilon_{N+1}^{2}\left(U_{0}\right)(1+t)^{\frac{-5}{2}},
$$

which implies (7.10). The proof of Proposition 7.0.2 is complete. 


\subsubsection{Decay rates in $L^{q}$}

In this subsection, we will prove Proposition 7.0.3 for time-decay rates in $L^{q}$ corresponding to (6.6)-(6.8) in Theorem 6.0.1. For $N \geq 4$, Proposition 7.0.2 shows that if $\epsilon_{N+1}\left(U_{0}\right)$ is small enough,

$$
\|U(s)\|_{N} \leq C \epsilon_{N+1}\left(U_{0}\right)(1+t)^{-\frac{3}{4}}
$$

and

$$
\|\nabla U(t)\|_{N} \leq C \epsilon_{N+1}\left(U_{0}\right)(1+t)^{\frac{-5}{4}}
$$

Now, let us establish the estimates on $\rho, u$, and $\left[c_{1}, c_{2}\right]$ in the following.

Estimate on $\|\rho(t)\|_{L^{q}}$. For the $L^{2}$ rate, it is easy to see from (10.5) and (10.9) that

$$
\|\rho(t)\| \leq C \epsilon_{N+1}\left(U_{0}\right)(1+t)^{\frac{-3}{4}} \leq C(1+t)^{\frac{-3}{4}} .
$$

For the $L^{\infty}$ rate, by applying the $L^{\infty}$ linear estimate on $\rho$ in (9.40) to the mild form (10.1), we have

$$
\begin{aligned}
\|\rho(t)\|_{\infty} \leq & C(1+t)^{\frac{-3}{2}}\left\|U_{0}\right\|_{L^{1}}+C e^{-\lambda t}\left\|\nabla^{2} U_{0}\right\|+C \int_{0}^{t}(1+t-s)^{\frac{-3}{2}}\left\|\left[g_{1},, g_{2}, g_{3}, g_{4}\right](s)\right\|_{L^{1}} d s \\
& +C \int_{0}^{t} e^{-\lambda(t-s)}\left\|\nabla^{2}\left[g_{1}, g_{2}, g_{3}, g_{4}\right](s)\right\| d s \\
& \leq C(1+t)^{\frac{-3}{2}}\left\|U_{0}\right\|_{L^{1} \cap \dot{H}^{2}}+C \int_{0}^{t}(1+t-s)^{\frac{-3}{2}}\left\|\left[g_{1}, g_{2}, g_{3}, g_{4}\right](s)\right\|_{L^{1} \cap \dot{H}^{2}} d s .
\end{aligned}
$$

Since by (10.9)

$$
\left\|\left[g_{1}, g_{2}, g_{3}, g_{4}\right](s)\right\|_{L^{1} \cap \dot{H}^{2}} \leq C\|\nabla U(t)\|_{N}\|U(s)\|_{N}+C\left\|_{N}\right\| U(s) \|_{N}^{2} \leq C \epsilon_{N+1}^{2}\left(U_{0}\right)(1+s)^{\frac{-3}{2}}
$$

putting the above inequality into (10.11), gives

$$
\|\rho(t)\|_{L^{\infty}} \leq C \epsilon_{N+1}\left(U_{0}\right)(1+t)^{\frac{-3}{2}}
$$

Then, by $L^{2}-L^{\infty}$ interpolation,

$$
\|\rho\|_{L^{q}} \leq C \epsilon_{N+1}\left(U_{0}\right)(1+t)^{\frac{-3}{2}+\frac{3}{2 q}}
$$

for $2 \leq q \leq \infty$.

Estimate on $\|u(t)\|_{L^{q}}$. For the $L^{2}$ rate, utilizing the $L^{2}$ estimate on $u$ in (9.39) to (10.1), we 
have

$$
\begin{aligned}
\|u(t)\| & \leq C(1+t)^{\frac{-5}{4}}\left\|U_{0}\right\|_{L^{1}}+C e^{-\lambda t}\left\|U_{0}\right\|+C \int_{0}^{t}(1+t-s)^{\frac{-5}{4}}\left\|g_{1}, g_{2}, g_{3}, g_{4}\right\|_{L^{1}} d s \\
& +\int_{0}^{t} e^{-\lambda(t-s)}\left\|\left[g_{1}, g_{2}, g_{3}, g_{4}\right](s)\right\| d s
\end{aligned}
$$

Due to (10.9),

$$
\left\|\left[g_{1}, g_{2}, g_{3}, g_{4}\right](s)\right\|_{L^{1} \cap L^{2}} \leq C\|U(s)\|_{N}^{2} \leq C \epsilon_{N+1}\left(U_{0}\right)(1+t)^{\frac{-3}{2}}
$$

Then (10.13) implies the slower decay estimate

$$
\|u(t)\| \leq C \epsilon_{N+1}\left(U_{0}\right)(1+t)^{\frac{-5}{4}} \leq C(1+t)^{\frac{-5}{4}} .
$$

For the $L^{\infty}$ rate, utilizing the $L^{\infty}$ estimate on $u$ in (9.41) to (10.1), we have

$$
\|u(t)\|_{L^{\infty}} \leq(1+t)^{-2}\left\|U_{0}\right\|_{L^{1} \cap \dot{H}^{2}}+C \int_{0}^{t}(1+t-s)^{-2}\left\|\left[g_{1}, g_{2}, g_{3}, g_{4}\right](s)\right\|_{L^{1} \cap \dot{H}^{2}} d s .
$$

Since by (10.9) and (10.10)

$$
\begin{aligned}
\left\|\left[g_{1}, g_{2}, g_{3}, g_{4}\right](s)\right\|_{L^{1} \cap \dot{H}^{2}} & \leq C\|\nabla U(t)\|_{N}\|U(s)\|_{N}+\|U(s)\|_{N}^{2} \\
& \leq C \epsilon_{N+1}^{2}\left(U_{0}\right)(1+s)^{-2}+C \epsilon_{N+1}^{2}\left(U_{0}\right)(1+s)^{\frac{-3}{2}} \\
& \leq C \epsilon_{N+1}^{2}\left(U_{0}\right)(1+s)^{\frac{-3}{2}} .
\end{aligned}
$$

It follows from (10.15) that

$$
\|u(t)\|_{L^{\infty}} \leq C \epsilon_{N+1}\left(U_{0}\right)(1+s)^{\frac{-3}{2}}
$$

Therefore, by $L^{2}-L^{\infty}$ interpolation,

$$
\|u(t)\|_{L^{q}} \leq C \epsilon_{N+1}\left(U_{0}\right)(1+t)^{\frac{-3}{2}+\frac{1}{2 q}}
$$

for $2 \leq q \leq \infty$.

Estimate on $\left\|c_{1}(t)\right\|_{L^{q}}$. For the $L^{2}$ rate, we utilize the $L^{2}$ estimate on $c_{1}$ in (9.42) to (10.1), we have

$$
\left\|c_{1}\right\| \leq C e^{-\lambda t}\left\|c_{1,0}\right\|_{L^{2}}+C \int_{0}^{t} e^{-\lambda(t-s)}\left\|g_{3}\right\| d s
$$


Since

$$
\left\|g_{3}(s)\right\| \leq C\|U(s)\|_{N}^{2} \leq C \epsilon_{N+1}\left(U_{0}\right)(1+t)^{\frac{-3}{2}} .
$$

Then (10.17) implies the slower decay estimate

$$
\left\|c_{1}\right\| \leq C \epsilon_{N+1}\left(U_{0}\right)(1+t)^{\frac{-3}{2}} .
$$

Similarly, we have

$$
\left\|c_{2}\right\| \leq C \epsilon_{N+1}\left(U_{0}\right)(1+t)^{\frac{-3}{2}} .
$$

For $L^{\infty}$ rate, we can utilize the $L^{\infty}$ estimate on $c_{1}$ in (9.44) to (10.1), we get

$$
\left\|c_{1}\right\|_{L^{\infty}} \leq C e^{-\lambda t}\left\|c_{1,0}\right\|_{L^{2} \cap \dot{H}^{2}}+C \int_{0}^{t} e^{-\lambda(t-s)}\left\|g_{3}\right\|_{L^{2} \cap \dot{H}^{2}} d s .
$$

From (10.9), we obtain

$$
\left\|g_{3}(s)\right\|_{L^{2} \cap \dot{H}^{2}} \leq C\|U(s)\|_{N}^{2} \leq C \epsilon_{N+1}\left(U_{0}\right)(1+t)^{\frac{-3}{2}},
$$

and thus

$$
\left\|c_{1}\right\|_{L^{\infty}} \leq C \epsilon_{N+1}\left(U_{0}\right)(1+t)^{\frac{-3}{2}} .
$$

Similarly, we have

$$
\left\|c_{2}\right\|_{L^{\infty}} \leq C \epsilon_{N+1}\left(U_{0}\right)(1+t)^{\frac{-3}{2}} .
$$

So, by $L^{2}-L^{\infty}$ interpolation,

$$
\left\|\left[c_{1}, c_{2}\right]\right\|_{L^{q}} \leq C \epsilon_{N+1}\left(U_{0}\right)(1+t)^{\frac{-3}{2}},
$$

for $2 \leq q \leq \infty$.

This completes the proof of Proposition 7.0.2 and hence Theorem 6.0.1. 


\section{References}

[1] J. Adler. Chemotaxis in bacteria. Science, 153(3737):708-716, 1966.

[2] M. A. J Chaplain and A. M. Stuart. A model mechanism for the chemotactic response of endothelial cells to tumor angiogenesis factor. MA J. Math. Appl. Med. Biol, 10(3):149$168,1993$.

[3] E. F. Keller and L. A. Segel. Initiation of slime mold aggregation viewed as an instability. J. Theor. Biol., 26:399-415, 1970.

[4] E. F. Keller and L. A. Segel. A model for chemotaxis. J. Theor. Biol., 2(30):225-234, 1971.

[5] Y. Wang. Boundedness in a three-dimensional attraction-repulsion chemotaxis system with nonlinear diffusion and logistic source. Journal of Differential Equations, 2016(176):1-21, 2016.

[6] E. Lankeit and J. Lankeit. Classical solutions to a logistic chemotaxis model with singular sensitivity and signal absorption. Math. AP, 2018.

[7] J. Liu and Z. Wang. Classical solutions and steady states of attraction-repulsion chemotaxis in one dimension. Journal of Biological Dynamics, 6:1-19, 2012.

[8] M. Chae, K. Kang, and J. Lee. Existence of smooth solutions to chemotaxis-fluid equations. Discrete and continuous dynamical systems, 33(6):2271-2297, 2013.

[9] R. Duan, A. Lorz, and Markowich P. The Cauchy problem on the compressible twofluids Euler-Maxwell equation. Milan J. Math., pages 1635-1673, 2010. 
[10] A. D. Rodriguez, L.C.F. Ferreira, and E. J. Villamizar-Roa. Global existence for an attraction-repulsion chemotaxis fluid model with logistic source. Math. AP, 2017.

[11] Z. Tan and J. Zhou. Global existence and time decay estimate of solutions to the KellerSegel system. Math Meth Appl Sci., 42:375-402, 2019.

[12] D. Ambrosi, F. Bussolino, and L. Preziosi. A review of vasculogenesis models. Theoretical Medicine, 6:1-19, 2005.

[13] R. J. Duan. Global smooth flows for the compressible Euler-Maxwell system: Relaxation case. Relaxation case, J. Hyperbolic Differential Equations, 8:375-413, 2011.

[14] R. J. Duan, Q. Liu, and C. Zhu. The Cauchy problem on the compressible two-fluids Euler-Maxwell equation. Milan J. Math., 44(1):102-133, 2012.

[15] A. Matsumura and T. Nishida. The initial value problem for the equations of motion of viscous and heat-conductive gases. Journal of Mathematics of Kyoto University, 20: 67-104, 1980.

[16] T. Kato. The Cauchy problem for quasi-linear symmetric hyperbolic systems. Arch Rational Mech Anal, 58:181-205, 1975.

[17] A. Majda. Compressible fluid flow and systems of conservation laws in several space variables. Applied Mathematical Sciences, 53,1984.

[18] M. Luca, Chavez-Ross, L. Edelstein-Keshet, and A. Mogilner. Chemotactic signaling, microglia, and Alzheimer's disease senile plaques: Is there a connection? Bulletin of Mathematical Biology, 65:693-730, 2003. 